\title{
MOLECULAR DYNAMICS CALCULATION OF THERMODIFFUSION COEFFICIENTS IN BINARY AND TERNARY MIXTURES
}

\author{
by \\ Seyedeh Hoda Mozaffari \\ Bachelor of Science, Mechanical Engineering, Iran University of Science \& Technology, \\ 2006 \\ Master of Science, Mechanical Engineering, University of Nevada, Las Vegas, USA, \\ 2011 \\ A dissertation \\ presented to Ryerson University \\ in partial fulfilment of the \\ requirements for the degree of \\ Doctor of Philosophy \\ in the program of \\ Mechanical \& Industrial Engineering \\ Toronto, Ontario, Canada, 2018 \\ (C) Seyedeh Hoda Mozaffari, 2018
}




\begin{abstract}
Author's Declaration
I hereby declare that I am the sole author of this dissertation. This is a true copy of the dissertation, including any required final revisions, as accepted by my examiners.

I authorize Ryerson University to lend this dissertation to other institutions or individuals for the purpose of scholarly research.
\end{abstract}

I further authorize Ryerson University to reproduce this dissertation by photocopying or by other means, in total or in part, at the request of other institutions or individuals for the purpose of scholarly research.

I understand that my dissertation may be made electronically available to the public.

Seyedeh Hoda Mozaffari 


\title{
Molecular Dynamics Calculation of Thermodiffusion Coefficients in Binary and Ternary mixtures
}

\author{
Doctor of Philosophy, 2018 \\ Mechanical \& Industrial Engineering \\ Ryerson University \\ Seyedeh Hoda Mozaffari
}

\begin{abstract}
Thermodiffusion phenomenon in fluid mixtures has been investigated by several scientists in theoretical as well as experimental fields for decades. Nevertheless, due to shortcomings of both methods, interest in searching for alternative approaches to shed some light on molecular scale of the phenomenon has spurred. The objective of this thesis is to develop an accurate molecular dynamics (MD) algorithm that can predict thermodiffusive separation in binary and ternary fluid mixtures. More importantly, the proposed algorithm should be computationally efficient in order to be suitable for integration into multi-scale computational models to simulate thermodiffusion in a large system such as an oil reservoir. In developing such an effective and efficient computational tool, this thesis introduces a modified heat exchange algorithms, wherein, a new mechanism is introduced to rescale velocities which curbs the energy loss in the system and at the same time minimizes the computational time. The performance of the new algorithm in studying Soret effect for binary and ternary mixtures has been compared with other non-equilibrium molecular dynamics (NEMD) models including regular heat exchange algorithm (HEX) and reverse non-equilibrium molecular dynamics (RNEMD). Different types of binary mixtures were studied including one equimolar mixture of argon (Ar)-krypton ( $\mathrm{Kr}$ ) above its triple point, non-equimolar normal alkane mixtures of hexane $\left(\mathrm{nC}_{6}\right)$-decane $\left(\mathrm{nC}_{10}\right)$ as well as hexane $\left(\mathrm{nC}_{6}\right)$-dodecane $\left(\mathrm{nC}_{12}\right)$ for six compositions, three non-equimolar mixtures of pentane $\left(\mathrm{nC}_{5}\right)$ decane $\left(\mathrm{nC}_{10}\right)$ at atmospheric temperature and pressure. Additionally, the new algorithm was validated for different ternary mixtures including ternary normal alkanes methane $\left(\mathrm{nC}_{1}\right)$-butane $\left(\mathrm{nC}_{4}\right)$ -
\end{abstract}


dodecane $\left(\mathrm{nC}_{12}\right)$ for three compositions, and one composition of different types of alkane mixture of 1,2,3,4-tetrahydronaphthalene (THN)-dodecane $\left(\mathrm{nC}_{12}\right)$-isobutylbenzene (IBB). The new algorithm demonstrates a significant improvement in reducing the energy loss by nearly $32 \%$. Additionally, the new algorithm is about $7-9 \%$ more computationally efficient than the regular HEX for medium and large systems. In terms of direction of thermodiffusive segregations in binary mixtures, in agreement with the experimental data, the new algorithm shows that the heavier component moves towards the cold region whereas the lighter component accumulates near the hot zone. Additionally, the strength of segregation process diminishes as the concentration of heavy component in the mixture increases. The new algorithm improved the prediction of thermodiffusion factor in binary mixtures by $24 \%$ in binary mixtures. With respect to the ternary mixtures, similarly to binary mixtures the heaviest and lightest component in the mixture move towards, cold and hot zones, respectively. While the intermediate component shows the least tendency to segregate. In terms of the strength of Soret effect, the new algorithm is about $17 \%$ more accurate than the regular HEX algorithm with respect to experimental data. 


\section{Acknowledgments}

I would like I would like to express my gratitude and sincere thanks to my supervisors, Prof. M. Z. Saghir and Dr. S. Srinivasan for their unequivocal support, patience, motivation, and immense knowledge.

Finally, I wish to thank my parents for their love and encouragement, without whom I would never have enjoyed so many opportunities. 


\section{Table of Contents}

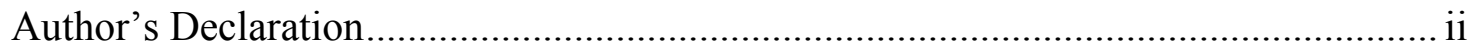

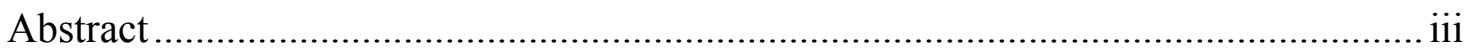

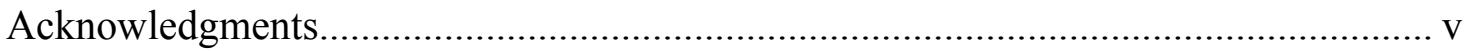

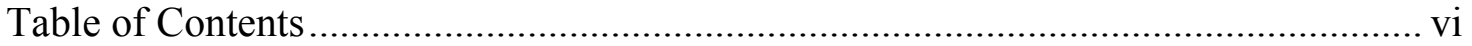

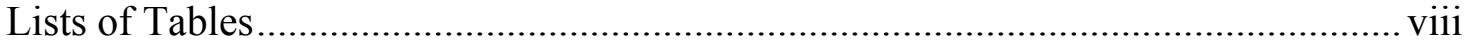

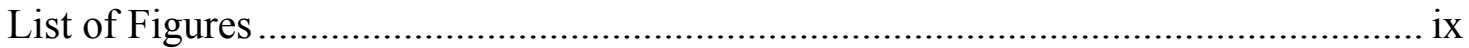

NOMENCLATURE ...................................................................................... xi

CHAPTER 1 - RESEARCH OBJECTIVES ........................................................... 1

1.1 History and General Views of Thermodiffusion ................................................. 1

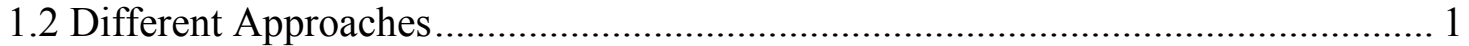

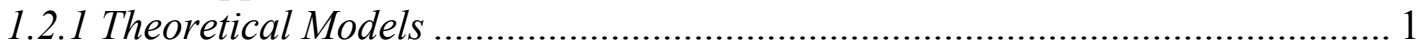

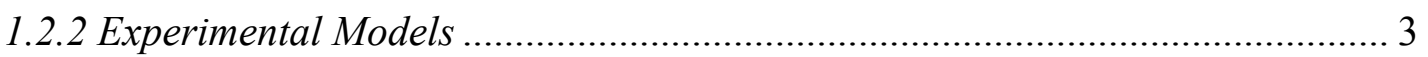

1.2.3 Computational Models ................................................................................. 4

1.2.4 Major Drawbacks of the Current Heat Generation Algorithm ........................ 7

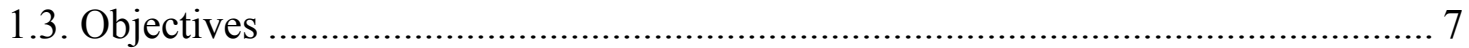

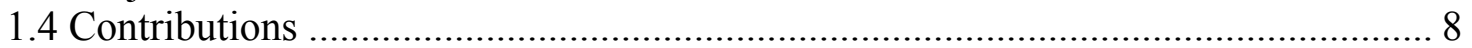

CHAPTER 2- EVALUATIONS OF MOLECULAR DYNAMICS METHODS FOR

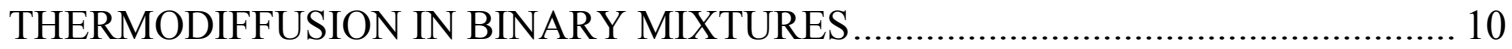

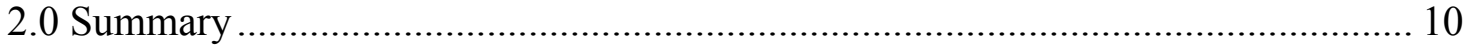

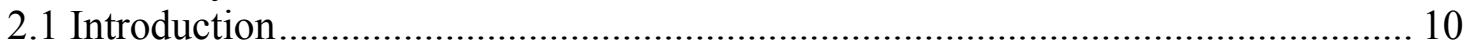

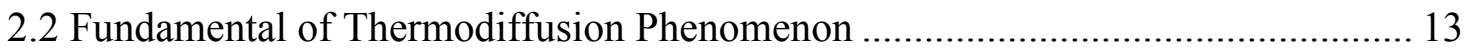

2.3 Details of MD Techniques .................................................................................. 14

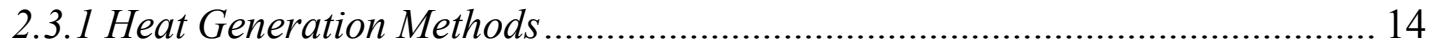

2.3.2 Pair Potential Functions and General MD Parameters ................................ 17

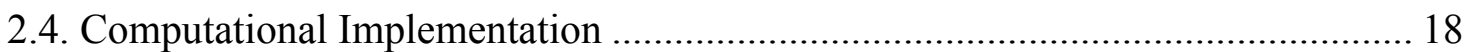

2.5. Computational Cases ..................................................................................... 19

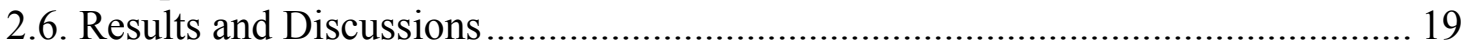

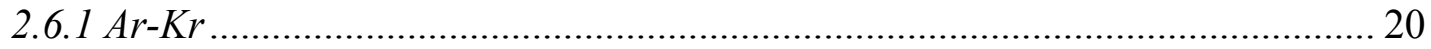

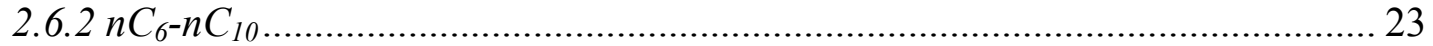

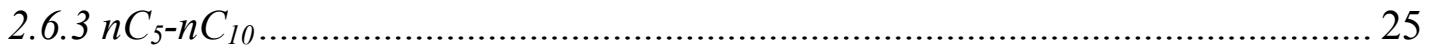

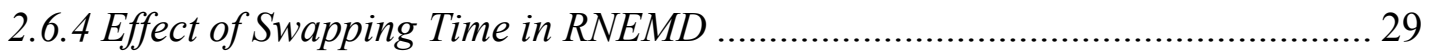

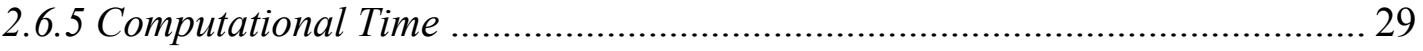

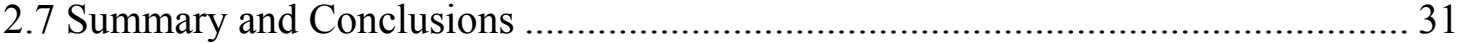

CHAPTER 3- THERMAL DIFFUSION IN BINARY AND TERNARY HYDROCARBON MIXTURES STUDIED USING A MODIFIED HEAT EXCHANGE

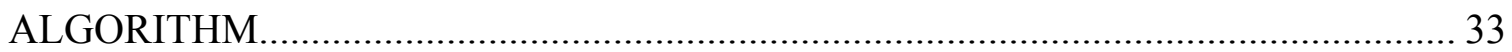

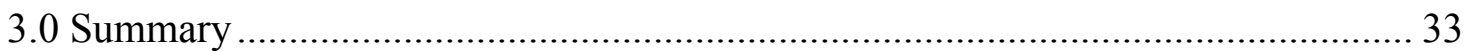

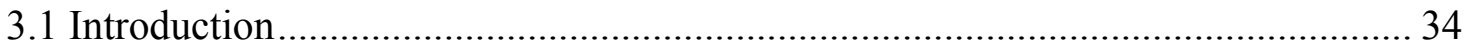

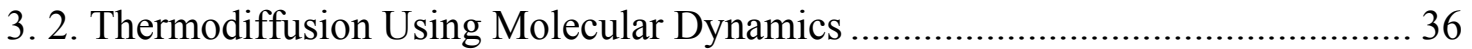

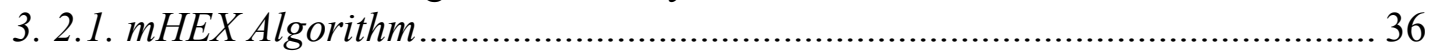

3. 2.2. Computational Implementation \& Details ................................................ 37

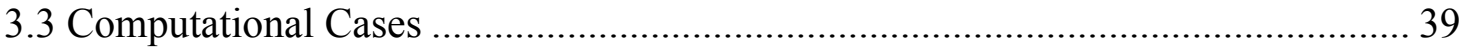




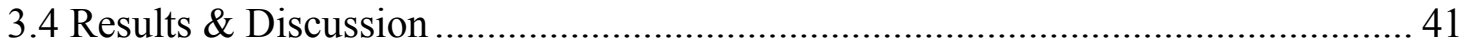

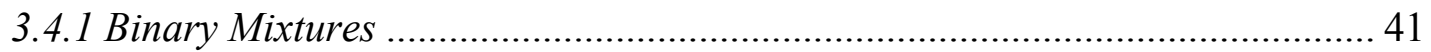

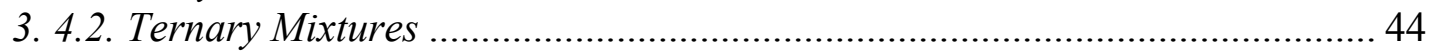

3. 5. Summary \& Conclusions .................................................................................... 48

CHAPTER 4- A MODIFIED HEAT EXCHANGE ALGORITHM TO STUDY THERMO-SOLUTAL DIFFUSION IN LIQUID MIXTURES ………….......................50

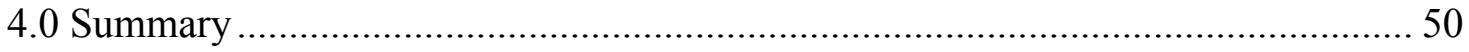

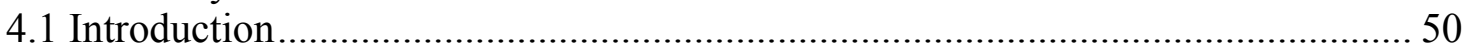

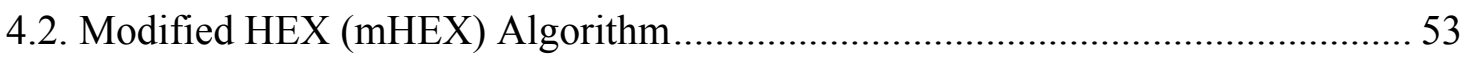

4.3. Computational Setup and Equilibration............................................................... 55

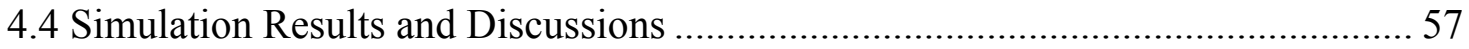

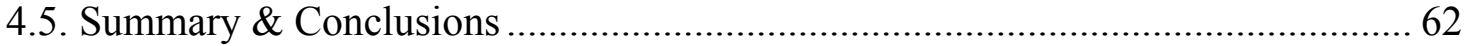

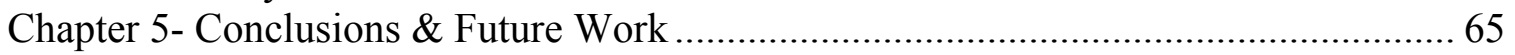

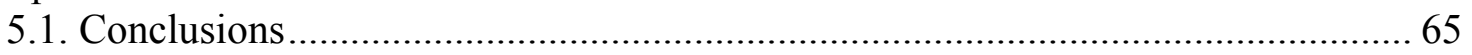

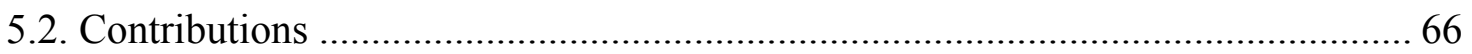

5.3. Future Work

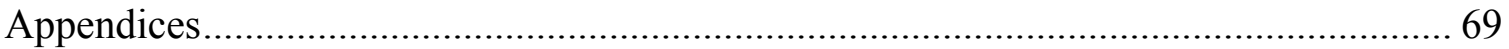

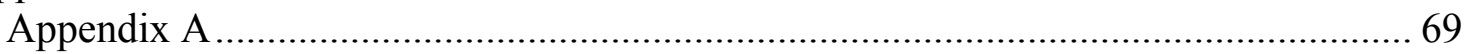

A.1 General Overview Molecular Dynamics............................................................ 69

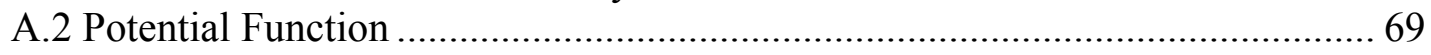

A.3 Integration Methods ................................................................................ 71

A.4 Periodic Boundary Condition...................................................................... 71

A.5 Calculation of Properties.............................................................................. 72

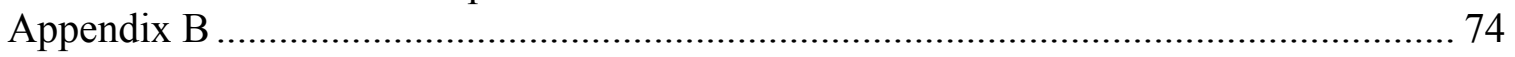

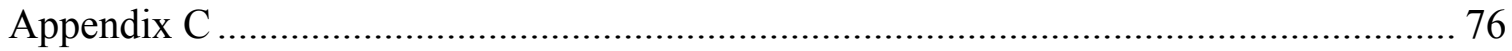

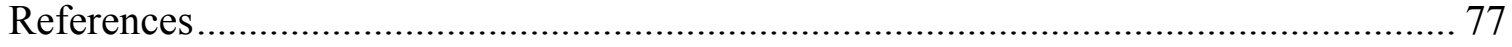




\section{Lists of Tables}

Table 2.1: Lennard-Jones potential parameters. These parameters were obtained from NIST Thermophysical Properties of Hydrocarbon Mixtures Database [123] ......... 18

Table 2.2: Predicted thermodiffusion factor for equimolar mixture of $\mathrm{Ar}-\mathrm{Kr}$ for $\mathrm{HEX}$, mHEX and RNEMD method with swapping time $=20$ iteration $[78]^{\mathrm{a}} \ldots \ldots \ldots \ldots \ldots \ldots . . . . . .22$

Table 2.3: Predicted thermodiffusion factor for equimolar mixture of $\mathrm{nC}_{6}-\mathrm{nC}_{10}$ for $\mathrm{HEX}$, MHEX and RNEMD method with swapping time $=20$ iteration $[78]^{\mathrm{a}} \ldots \ldots \ldots \ldots \ldots \ldots . . . . . .25$

Table 2.4: Predicted thermodiffusion factor for three different mixtures $\mathrm{nC}_{5}-\mathrm{nC}_{10}$ for HEX, mHEX. Numbers in the parentheses indicate the relative error with respect to the experimental data $[78]^{\mathrm{a}}$.

Table 2.5: Computational time of per 1000 iterations for HEX, mHEX and RNEMD

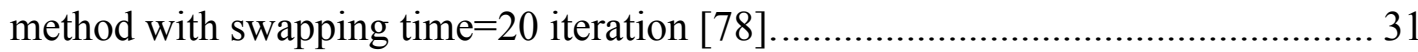

Table 3.1: Lennard-Jones potential parameters obtained from Ref. [123, 138]. 39

Table 3.2: Binary and ternary mixtures for which MD simulations were performed. All compositions are in mole fractions except for mixture \#14 for which the composition

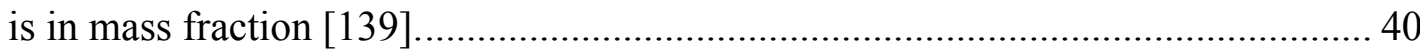

Table 3.3: Thermodiffusion factor of the binary mixtures [139] ................................. 44

Table 3.4: Thermodiffusion factors in $\mathrm{nC} 1-\mathrm{nC} 4-\mathrm{nC} 12$ mixtures. The experimental data is from Srinivasan and Saghir [134]. The HEX results are from the work of Galliero et al. [85]. The mHEX results are from Mozaffari et al. [139] ${ }^{\mathrm{a}}$ 46

Table 3.5: Soret Coefficients $(1 / \mathrm{K}) \times 10^{4}$ in $\mathrm{nC}_{12}$-IBB-THN mixture. The experimental data is from Ahadi and Saghir [132]. The HEX and mHEX are from Mozaffari et al. $[139]^{\mathrm{a}}$

Table 4.1: Lennard-Jones potential parameters. These parameters were obtained from NIST Thermophysical Properties of Hydrocarbon Mixtures Database [123] ......... 55

Table 4.2: Thermodiffusion factor estimated using the mHEX and HEX algorithm on a system of 1000 particles. The value in parentheses indicates the deviation from the experimental/ benchmark data in the reported reference. 


\section{List of Figures}

Fig 1.1: A schematic of a Soret cell [1]................................................................... 3

Fig 1.2: A schematic of Thermogravitational column [1] ........................................... 4

Fig. 2.1: Schematic view of simulation box [78] ................................................... 18

Fig. 2.2: Dimensionless temperature distribution inside the simulation box for equimolar mixture of Ar-Kr using the HEX, RNEMD (with swapping time $=20$ time step) and mHEX algorithms [78].

Fig. 2.3: $\mathrm{Kr}$ concentration profile inside the simulation box for equimolar mixture of Ar$\mathrm{Kr}$ using the HEX, RNEMD (with swapping time $=20$ time step) and MHEX algorithms [78]. 21

Fig. 2.4: Ar concentration profile inside the simulation box for equimolar mixture of Ar$\mathrm{Kr}$ using the HEX, RNEMD (with swapping time $=20$ time step) and MHEX algorithms [78]. 22

Fig. 2.5:Average dimensionless temperature distribution in middle layers for nonequimolar $\mathrm{nC}_{6}-\mathrm{nC}_{10}$ mixture using the $\mathrm{HEX}, \mathrm{RNEMD}$ (with swapping time $=20$ time step) and MHEX algorithms [78]. 23

Fig. 2.6: Average mole fraction trend of $\mathrm{nC}_{10}$ in middle layers for non-equimolar $\mathrm{nC}_{6^{-}}$ $\mathrm{nC}_{10}$ mixture using the HEX, RNEMD (with swapping time $=20$ time step) and mHEX algorithms [78].

Fig. 2.7: Average mole fraction trend of $\mathrm{nC}_{6}$ in middle layers for non-equimolar $\mathrm{nC}_{6}-\mathrm{nC}_{10}$ mixture using the HEX, RNEMD (with swapping time $=20$ time step) and MHEX algorithms [78]

Fig. 2.8:Average dimensionless temperature distribution in middle layers for $\mathrm{nC}_{5}-\mathrm{nC}_{10}$ mixture with an initial uniform mole fraction of $\mathrm{nC}_{5}=0.8$, using the $\mathrm{HEX}$, and MHEX algorithms [78].

26

Fig. 2.9:Average mole fraction trend of $\mathrm{nC}_{10}$ in middle layers for $\mathrm{nC}_{5}-\mathrm{nC}_{10}$ mixture with an initial uniform mole fraction of $\mathrm{nC}_{5}=0.8$, using the $\mathrm{HEX}$, and $\mathrm{MHEX}$ algorithms [78].

Fig. 2.10:Average mole fraction trend of $\mathrm{nC}_{5}$ in middle layers for $\mathrm{nC}_{5}-\mathrm{nC}_{10}$ mixture with an initial uniform mole fraction of $\mathrm{nC}_{5}=0.8$, using the HEX, and MHEX algorithms [78].

Fig. 2.11: Thermodiffusion factor versus velocity swapping time for RNEMD method [78]. 30

Fig. 3.1: Computational domain subdivided into slabs [140] ....................................... 43

Fig. 3.2: $(\mathrm{a}, \mathrm{b})$ Typical temperature distribution in the domain in the $\mathrm{nC}_{6}-\mathrm{nC}_{10}$ and $\mathrm{nC}_{6^{-}}$ $\mathrm{nC}_{12}$ mixtures, respectively[140]. 45

Fig. 3.3: $(\mathrm{a}, \mathrm{b})$ Distribution of $\mathrm{nC}_{6}$ in the domain in the $\mathrm{nC}_{6}-\mathrm{nC}_{10}$ and $\mathrm{nC}_{6}-\mathrm{nC}_{12}$ mixtures, respectively. In both mixtures, the mole fraction of $\mathrm{nC}_{6}=0.9$ [140] .................... 46

Fig. 3.4: Thermodiffusion factors in the binary mixtures \#11-13 [140] ........................ 47

Fig. 3.5: Temperature distribution in the domain in mixture $\# 11$ [140]......................... 48

Fig. 3.6: $(\mathrm{a}, \mathrm{b})$ Distribution of $\mathrm{nC}_{12}$ and $\mathrm{nC}_{4}$, respectively, in the domain in mixture \#11

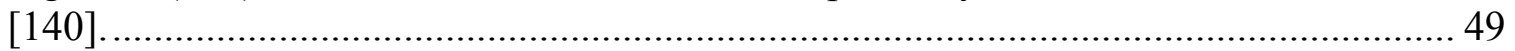

Fig. 3.7: Thermodiffusion factors in the ternary mixtures $\# 11-13$ [140]....................... 50

Fig. 3.8: Temperature distribution in the domain in mixture \#14 [140] ........................ 51 
Fig. 3.9: $(a, b)$ Distribution of IBB and THN, respectively, in the domain in mixture \#14

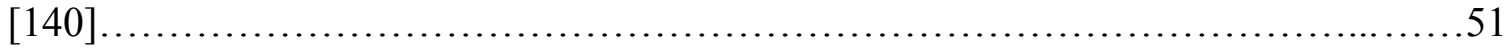

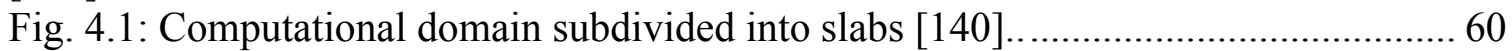

Fig. 4.2: Value of $\zeta$ in Ar-Kr mixture using the mHEX and HEX algorithms................ 62

Fig. 4.3: Magnitude of cumulative energy loss for the $\mathrm{MD}$ simulation of the $\mathrm{nC}_{5}-\mathrm{nC}_{10}$ mixture with mole fraction of $\mathrm{nC}_{5}=0.2$ from the mHEX and HEX algorithms....... 64

Fig. 4.4: Magnitude of total energy loss at the of the simualtion as a function of number

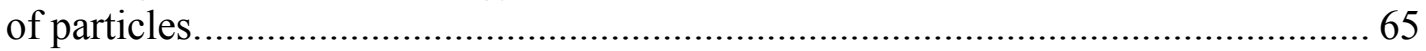

Fig. 4.5: Thermodiffusion factor calculated using mHEX algorithm as a function of the

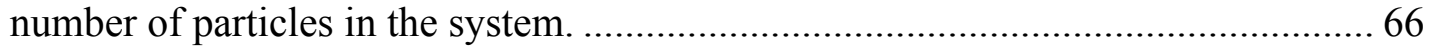

Fig. 4.6: Savings in the computational time as a function of the size of the system. Dotted

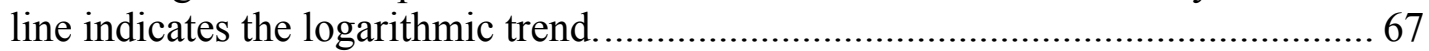




\section{NOMENCLATURE}

\begin{tabular}{|c|c|}
\hline$m$ & Mass, (kg) \\
\hline$r$ & Distance between particles and position of particle, $\mathrm{m}$ \\
\hline$t$ & Time, s \\
\hline$k_{b}$ & Boltzmann constant, $\mathrm{J} / \mathrm{K}$ \\
\hline$x$ & Mole Fraction \\
\hline C & Concentration, mole $/ \mathrm{m}^{3}$ \\
\hline$D$ & Molecular diffusion coefficient, $\mathrm{m}^{2} / \mathrm{s}$ \\
\hline$D_{T}$ & Thermodiffusion coefficient, $\mathrm{m}^{2} /(\mathrm{s} . \mathrm{K})$ \\
\hline$J_{l}$ & Mole flow rate, mole $/\left(\mathrm{m}^{2} . \mathrm{s}\right)$ \\
\hline$J_{q}$ & Internal energy flux, $\mathrm{J} /\left(\mathrm{m}^{2} . \mathrm{s}\right)$ \\
\hline$L_{i j}$ & Phenomenological coefficient \\
\hline$N$ & Number of particles \\
\hline$T^{*}$ & Dimensionless temperature \\
\hline$U$ & Energy removal or addition, $(\mathrm{J})$ \\
\hline$V$ & Velocity, $\mathrm{m} / \mathrm{s}$ \\
\hline$V_{b}$ & Barycentric velocity, $\mathrm{m} / \mathrm{s}$ \\
\hline \multicolumn{2}{|c|}{ Greek Symbols } \\
\hline$\alpha$ & Thermodiffusion factor \\
\hline$\zeta$ & Scaling Factor \\
\hline$\gamma$ & Scaling Factor \\
\hline$\varepsilon$ & Depth of Potential Well, J \\
\hline$\mu$ & Chemical potential, $\mathrm{J} / \mathrm{mole}$ \\
\hline$\rho^{*}$ & Dimensionless Density \\
\hline$\sigma$ & Atomic diameter, nm \\
\hline$\phi$ & Lennard-Jones potential, $\mathrm{J}$ \\
\hline \multicolumn{2}{|c|}{ Subscripts } \\
\hline 1 & Component 1 , heavy \\
\hline 2 & Component 2, light \\
\hline$c$ & Cold region \\
\hline$h$ & Hot region \\
\hline$i, j$ & Particle type \\
\hline$k$ & Counting number \\
\hline$T$ & Thermal diffusion \\
\hline \multicolumn{2}{|c|}{ Notations } \\
\hline$\nabla$ & Spatial derivative \\
\hline
\end{tabular}




\section{CHAPTER 1- RESEARCH OBJECTIVES}

\subsection{History and General Views of Thermodiffusion}

Carl Ludwig, German chemist and physician was the first person noticed the impact of temperature nonuniformity on an isotropic liquid mixture in 1856 [1]. The Swiss physicist and chemist Charles Soret described the same phenomenon in further details 23 years later [2]. He discovered that when salt solutions of $\mathrm{NaCl}$ and $\mathrm{KNO}_{3}$ confined in tube shape containers were subject to different temperatures at the two ends, the solutions were not remained even in composition. After repeating the experiment with different salt solutes including $\mathrm{KCL}, \mathrm{LiCl}$ and $\mathrm{CuSO}_{4}$, he concluded that salt always concentrated at the cold end [3,4]. This coupled mass and heat transfer phenomenon has been addressed in literature with different names like thermodiffusion, thermophoresis, thermotransport and thermomigration. However, it is often known as Soret effect to honour Charles Soret's extensive work on the subject including formulation of governing equations.

The thermodiffusive segregation has been observed and studied in several types of mixtures, viz., gases [5-7], electrolytes [8,9], alcohols [10-12], polymers [13-15], molten metals [16], ferrofluids [17-19], semiconductor materials [20-22], latex particles [23] and proteins $[24,25]$. The strength of the Soret effect, as well as its direction is usually determined by a parameter called Soret coefficient, $S_{T}\left(\mathrm{~K}^{-1}\right)$ [26]. The order of magnitude

of Soret coefficient usually is less than $10^{-2} \mathrm{~K}^{-1}$ [26]. Nonetheless, the impact of thermodiffusion on numerous natural activities such as oceanic thermohaline circulation [27] and convection in stars [28] is crucial.

\subsection{Different Approaches}

\subsubsection{Theoretical Models}


Several theoretical models have been developed to investigate Soret effect in different mixtures, though these models first arrived almost a century after the discovery of the phenomenon. In general, scientists have better understanding of the thermodiffusion in gaseous mixtures based on kinetic theory of gases than non-ideal fluid mixtures [29]. Drickamer and his team [30-34] were pioneers in proposing different theories for thermal diffusion in non-ideal mixtures based on linear non-equilibrium thermodynamics (LNET) principles in 50s. In these models, it is assumed that infinitesimal volume elements of an irreversible system are in locally equilibrium condition. As a result, the classical thermodynamics relations can be applied to these elementary volumes; which leads to emerge of an energy quantity named 'net heat of transport'. The LNET models have puzzled researchers for decades to define the new quantity in terms of measurable thermodynamic properties.

Apart from these, other early predominant theoretical studies on comprehending the Soret effect are the ones proposed by Haase [35], Moritmer and Eyring [36], Guy [37], and Kempers [29, 36]. Hasse's model for thermal diffusion was derived from analogy of mass transfer due to pressure gradient for binary electrolyte mixture. Moritmer and Eyring [36] suggested an equation for probability of an individual type of molecule per time to jump from an old equilibrium state to a new equilibrium state for binary mixtures of molecules with equal size. In Guy's LNET model [37], Soret coefficients were formulated as a function of partial molar excess energy of pure components. While Kempers' $[29,38]$ models were based on the principles of statistical nonequilibrium thermodynamics.

Moreover, the first attempts to explain thermophoresis activities in dilute binary fluid mixtures via hydrodynamic/ Brownian motion models were done by Brenner and his colleague [39-41]. The essence of their hydrodynamic approach is based on the volume transport theory suggested by Brenner [42], in which a non-zero diffusive volume flux accompanies the Fourier heat flux even when the fluid is at rest, i.e. mass flux is zero. According to his models, thermal diffusivity of a dilute solution only depends on solvent's properties. 
In recent years, the researchers' focus in theoretical field has shifted to extend and modify previous LNET theoretical models to different types of mixtures including associating [43] and non-associating mixtures [44], DNA solutions [45], polymers [46] as well as ternary and quaternary mixtures $[47,48]$. Several detailed reviews about these theories can be found in the literature $[49,50]$.

\subsubsection{Experimental Models}

In parallel attempts, scientists have conducted various experimental approaches to measure thermodiffusion coefficients in a multicomponent fluid mixture [51-56]. The presence of gravity has a significant impact on thermodiffusion phenomenon. As a result, the earliest empirical techniques can be categorized differently based on the permissibility of convection. Here two primitive setups with different configurations will be explained. Fig. 1.1 illustrates the first common configuration (Soret cell), where liquid mixture is confined in a thin space between two horizontally separated plates that are kept at different temperatures. The objective of experimental set ups with this configuration is to elude any convectional fields [51]. Later, to evaluate Soret coefficients the change in density or refractive index of fluid mixture is studied to obtain concentration profile.

\begin{tabular}{|c|c|}
\hline Plate 1 & $\mathrm{~T} 1$ \\
\hline \multicolumn{2}{|c|}{ Fluid Mixture } \\
\hline Plate 2 & $\mathrm{~T} 2$ \\
\hline
\end{tabular}

Fig. 1.1: A schematic of a Soret cell [1].

Thermogravitational column is the second common configuration, which was first introduced by Clusius and Dickel [52] (c. f. Fig. 1.2). Where the fluid mixture is enclosed in a small space between two walls at different temperatures. According to Soret effect, the lateral temperature gradients in this configuration create lateral density gradients; which introduce a gravitational convection field along the testing cell. In other words, a 
combination of thermal, gravitational and buoyancy fields affect the segregation process. In order to measure Soret coefficient, two distinct methodologies can be used. In the first method, extracting fluid samples from the column at different heights to analyse the concentration profile along the cell after reaching to steady state condition. Later, Dutrieux et al [53] introduced a new methodology to quantify Soret coefficient via using laser Doppler velocimetry (LDV) to record the magnitude of mixture's velocity at different time.

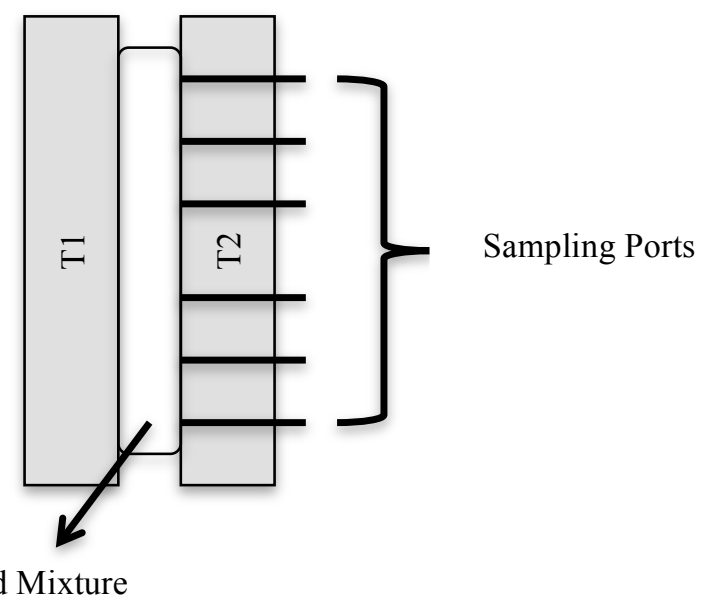

Fig. 1.2: A schematic of Thermogravitational column [1].

With several such apparatus and experimental techniques in the literature, in general, experimental techniques can be classified into two major types, viz. optical and nonoptical methods. Optical approaches like Soret cell with beam deflection (BD) [54], thermal diffusion forced Rayleigh scattering (TDFRS) [55], microfluidic fluorescence [56], and thermal lens model [57] are generally more complicated and expensive. The famous non-optical methods are classical Soret cell [51], two-chamber thermodiffusion cells [58], thermal field-flow fractionation [59], and thermogravitational column approaches $[53,54]$. In literature, several comprehensive reviews have addressed the weak and strong aspects of these empirical aforementioned models $[26,50,60]$.

\subsubsection{Computational Models}

Given some inherited deficiencies and shortcomings of theories as well as experimental methods, computational approaches can be used as potential substitutes to study the 
thermodiffusive flows. On the theoretical front, the choice of equation of states (EOS), and proper calculation of the thermodynamic properties of pure components and mixture can alter the results significantly [61]. Therefore, theoretical models are not often in good agreement with each other on the strength and even sign of thermodiffusion coefficients.

On the other hand, several external sources of errors including undesired natural convection fields, mechanical vibrations as well as post-processing errors compromise the accuracy of experimental methods [62-64]. In fact, in view of these experimental error sources, recently, there has been a significant surge in conducting the thermodiffusion experiments on reduced-gravity environment to investigate the impact of vibrations and minimalize the unwanted effect of gravity [65-68]. However, these are prohibitively expensive and are experiments have to be planned years in advance because of limited access to reduced gravity environment.

As a compromise, inexpensive molecular dynamics (MD) techniques, that is also the focus of this thesis, can play a significant role to bridge a comprehensive approach between molecular scale and macroscopic characteristics of the phenomenon among other suggested numerical methods including artificial neural networks [69]. The application of MD models to comprehend the thermodiffusive properties of fluid mixture dates back to 1980s and 1990s, where three famous non-equilibrium molecular dynamic (NEMD) techniques, i.e. synthetic non-equilibrium molecular (SNEMD), heat exchange algorithm (HEX) as well as reverse non-equilibrium molecular dynamics (RNEMD), were established.

In 1986, Evans and MacGowan [70] introduced a technique called synthetic nonequilibrium molecular dynamics (SNEMD) to investigate thermodiffusion in an equimolar liquid argon-krypton mixture through generalization of his previous methodologies for measuring self-diffusion [71] and thermal conductivity [72] of a one component liquid system. The system in these algorithms is subjected to specific type of time-variable external forces that result in disturbance and deviation from equilibrium conditions in phase space. Later, phenomenological coefficients through application of 
linear response theory and auto-correlation functions can be computed which leads to estimation of thermodiffusion factor.

In early 90s Hafskjold $[73,75]$ and his team established a popular and straightforward direct non-equilibrium molecular dynamics (DNEMD) approach to calculate Soret coefficients directly via rescaling velocities. In their well-known heat exchange algorithm (HEX) [73], the simulation domain is divided into three main zones: hot, middle and cold regions. They developed a methodology to introduce heat flux into the system via exchanging certain amount of energy between cold and hot zones without violation of conservation of momentum and total energy. As a result, linear distributions of temperature and concentration will be introduced to the system. Later, the Soret coefficient can be computed directly based on the ratio of the slope of these aforementioned distributions. The main target of Hafskjold and his colleagues [73] studies was to investigate equimolar isotope liquid mixtures and heat conduction near liquid-gas interface as well as real equimolar argon-krypton mixture.

Reverse non-equilibrium molecular dynamics approach (RNEMD) is another well-known DNEMD technique that was introduced by Müller-Plathe [74] in 1997.The initial objective of this method like its preceding D-NEMD algorithm was to measure thermal conductivity of a pure liquid; however, it can be beneficial to predict mixture transport properties in a liquid mixture. The simulation box like HEX technique is divided into different slabs, which form the hot, middle and cold regions. In spite of former approach the establishment of heat flux in the system is done through a straightforward swapping of particles' velocities with the equal mass in hot and cold layers.

In general, SNEMD approaches have been largely superseded by DNEMD techniques, since in these methods the transport coefficient can be estimated directly without calculation of phenomenological coefficients. Additionally, despite RNEMD clarity and easiness, this technique has not gained the popularity of HEX method. The original and affiliated RNEMD techniques often have shown large error margins in predicting Soret coefficients with respect to experimental data [76-78] in the literature. The essence of 
heat flux generation in this method; which involve with sudden swapping particle's velocities may contribute to these relatively large errors.

\subsubsection{Major Drawbacks of the Current Heat Generation Algorithm}

In studying Thermodiffusion using Molecular Dynamics, several recent studies have employed the HEX algorithm to estimate thermodiffusive properties of different types of fluid mixtures including isotopes [79-81] and hydrocarbons [83-85]. However, there are several drawbacks of this HEX algorithm:

1. The accuracy of the HEX results is highly dependent upon the size the system. More precisely, for small and medium systems the algorithm often fails to generate a stable heat flux [86].

2. The HEX algorithm demonstrates significant energy drifts due to its leading order truncation errors and fluctuating scaling factors. In order to restrain these losses energy losses smaller time steps can be used which results into more time consuming simulation $[87,88]$.

3. Scaling factor in HEX algorithm must be calculated in each time step inside the main loop for hot and cold zone which makes the system computationally inefficient. More precisely, there is a $\mathrm{O}\left(\mathrm{N}^{2}\right)$ calculation that is to be made at every time step, $\mathrm{n}$ being the number of molecules in the system.

As a consequence of these drawbacks the algorithm cannot be employed to study largescale systems such as an entire reservoir or even in a multi-scale format to study a relatively smaller section of a reservoir.

\subsection{Objectives}

In this thesis, we use principles of molecular dynamics to study Thermodiffusion in binary and ternary mixtures. In doing so, noting the shortcomings of not only the other theoretical approaches and experimental methods but also of the HEX algorithm used in MD simulations in studying the large-scale thermodiffusive separation behaviour, the main objectives of this research are as follows: 
1- Study the Soret effect in molecular level via consideration of interactions between particles for binary and ternary liquid mixtures.

2- Develop a computationally efficient and accurate MD simulation tool that can be integrated to multi-scale computational models to simulate thermodiffusion in a large system like oil reservoirs.

3- Evaluate the tool with respect to current commonly used heat generation algorithms, i.e. reverse non-equilibrium molecular dynamics (RNEMD) and heat exchange algorithm (HEX).

4- Improve the HEX algorithms in terms of energy stability as well as computational efficiency.

5- Investigate the impact of the size of the system, i.e., the number of particles in the system, on the performance of the modified algorithms for different binary and ternary mixtures.

\subsection{Contributions}

In realizing the above objectives, the following major contributions have been made in this research:

1. The velocity rescaling mechanism in the traditional heat exchange algorithm was reviewed and it was completely modified in the new algorithm. More precisely, a constant rescaling factor was introduced in place of the rescaling equations (c.f. Eqns. (2.5) and (2.6)), reducing the number of calculations by $\mathrm{O}\left(\mathrm{N}^{2}\right)$ in each iterations. The revised algorithm was employed to study Thermodiffusion in 5 binary mixtures. It was also compared with respect to RNEMD, HEX and experimental data. Overall, it has been shown that the modified algorithm proposed in this thesis is nearly $14 \%$ and $8 \%$ more accurate than RNEMD and HEX algorithms, respectively in predicting thermodiffusion for binary mixtures. The findings from this are published in ASME Journal of Thermal Science Engineering and Applications and details from this publication are given at the end of this chapter as well as at the beginning of Chapter 2. 
2. Subsequent to the above modification, as a major improvement, instead of using a constant value for the velocity rescaling factor, an expression in terms of mixture properties, namely, atomic parameters, temperature and density of mixture was proposed to calculate the scaling factor (c.f. Eqn. (3.3)). Note that this revised expression is not the same or even similar to the Eqns. (2.5) and (2.6) referred in the previous point. The modified HEX algorithm equipped with this revised expression was evaluated with respect to 14 binary and ternary hydrocarbon mixtures. It has been shown that the new algorithm suggested in this thesis is 17 $\%$ more accurate than HEX algorithm to predict thermodiffusion in ternary mixtures. It must be noted that the experimental results were obtained from microgravity environment. The findings from this are published in Journal of Thermal Science and Engineering in Progress and details from this publication are given at the end of this chapter as well as at the beginning of Chapter 3.

3. The energy conservation and computational time of the proposed algorithm in this thesis were compared with HEX algorithm for 6 different binary mixtures. The performance of both systems with respect to the size of the system was studied. It has been shown that the computational speed is nearly $9 \%$ faster for modified algorithm than HEX for large systems. Additionally, the modified algorithm has improved the energy drift by $30 \%$. Details pertaining to this are submitted to be published with the International Journal of Thermal Sciences. 


\section{CHAPTER 2- EVALUATIONS OF MOLECULAR DYNAMICS METHODS FOR THERMODIFFUSION IN BINARY MIXTURES}

This chapter is based on the following published paper:

Mozaffari, S. H., Srinivasan, S. \& Saghir, M. Z.,

Evaluations of molecular dynamics methods for thermodiffusion in binary mixtures,

ASME J. Therm. Sci. Eng. Appl., 9 (3) (2017), 031011-1-9.

\subsection{Summary}

The objective of this work is to investigate the behavior of two well-known boundary driven molecular dynamics (MD) approaches, namely, reverse non-equilibrium molecular dynamics (RNEMD) and heat exchange algorithm (HEX), as well as introducing a modified HEX model (mHEX) that is more accurate and computationally efficient to simulate mass and heat transfer mechanism. For this investigation, the following binary mixtures were considered: one equimolar mixture of argon (Ar)-krypton $(\mathrm{Kr})$, one non-equimolar liquid mixture of hexane $\left(\mathrm{nC}_{6}\right)$ and decane $\left(\mathrm{nC}_{10}\right)$, and three non-equimolar mixtures of pentane $\left(\mathrm{nC}_{5}\right)$ and decane $\left(\mathrm{nC}_{10}\right)$. In estimating the Thermodiffusion factor in these mixtures using the three methods, it was found that consistent with the findings in the literature, RNEMD predictions have the largest error with respect to the experimental data. Whereas, the mHEX method proposed in this work is the most accurate, marginally outperforming the HEX method. Most importantly, the computational efficiency of mHEX method is the highest, about $7 \%$ faster than the HEX method. This makes it more suitable for integration with multi-scale computational models to simulate Thermodiffusion in a large system such as an oil reservoir.

\subsection{Introduction}

A spatial inconsistency of temperature in a homogeneous gaseous or liquid mixture in the absence of free convection is a driving force for a coupled mass and heat transport phenomenon, which is called thermodiffusion (Soret effect) [89]. In other words, the temperature gradient develops a unique separation direction for each component in the 
mixture. The strength of the Soret effect, as well as its direction is usually characterized by a parameter called thermodiffusion factor, $\alpha_{T}$. Thermodiffusion has several industrial applications including isotope segregation in fluid mixture [90], freezing food processing [91] and polymer characterization [92]. Additionally, Soret effect influences various natural phenomena like salinity gradient in the ocean [93], physical concept of solar ponds [94] and distinct compositional variation of constituents in hydrocarbon reservoirs [95]. Given the very subtle nature of this phenomenon, the precise estimation of thermodiffusion factor has been a challenge for experimentalists for decades.

On the other hand, theoreticians' attempts to develop an explanation for this phenomenon have led to numerous theoretical models. These theoretical techniques can be classified in different ways including "static models" versus "dynamic theories" [96] and "matching parameter models" versus "independent methods" [49]. The kinetic gas theory and its alterations [97, 98], kinetic and phenomenological theories of irreversible thermodynamics [31, 37, 99-102], transition state theory [36], hydrodynamic and Brownian motion model [103, 104], statistical non-equilibrium thermodynamics [29] are principles of theoretical methods in obtaining thermodiffusion factor. A comprehensive review of these theoretical models is presented by Saghir and Eslamian [49].

Nevertheless, both theoretical models and empirical techniques are often in disagreement on the strength of thermodiffusive separation. These can be attributed to factors such as the choice of equation of state (EOS) and thermodynamic properties of pure components and mixture used in the calculations [61]. On the experimental front, errors can creep in due to the several external factors including natural gravity, mechanical vibrations and the handling of the mixtures constituents during the post-processing of the experiment [63, 105-107]. More recently, the use of artificial neural networks to study thermodiffusion in liquid mixture has been suggested $[69,108,109]$.

Among other computational techniques, molecular dynamics (MD) serves as a substantial numerical method and a low-cost alternative for experiments. The MD methods used to estimate transport properties of a mixture can be broadly divided into 
two major categories: equilibrium molecular dynamics (EMD) [110, 111] and nonequilibrium molecular dynamics (NEMD) approaches [112-114]. In the former method the transport properties can be calculated through Green-Kubo or Einstein formula, which links the integral of auto-correlation of flow quantities to corresponding dynamic properties in the absence of any agitating fields. The later technique computes the dynamic properties of the system in the presence of external forces or perturbed fields. NEMD method comprises of the synthetic (SNEMD) [70, 115, 116], boundary driven (direct) approaches including heat exchange algorithm (HEX) [73, 74] and reverse (RNEMD) [75, 117] approaches to predict thermodiffusive separation.

SNEMD methods are not capable of measuring thermodiffusion factor directly, and calculate phenomenological coefficient instead. As per this algorithm, the system is subjected to specific time-varying external forces that induce a disturbance and deviation from equilibrium conditions in phase space. Subsequently, phenomenological coefficients are computed by applying the linear response theory and auto-correlation functions that can be used to estimate thermodiffusion factor. In more recent times, the SNEMD algorithms have been replaced by direct approaches in which thermodiffusion can be estimated directly without calculation of phenomenological coefficients. Among different boundary driven techniques, heat-exchanging algorithm (HEX) [73] has gained popularity. Many researchers adopted the HEX and RNEMD algorithms to predict thermodiffusion factor for different mixtures [80, 83-85, 118, 119]. However, there is a dearth of comprehensive reviews to compare these techniques in the literature.

In presenting our work in this direction, the rest of the paper is organized as follows: In Section 2.2, the underlying theoretical formalism of the Thermodiffusion phenomenon is presented. In Section 2.3, the details of the molecular dynamics algorithms to study Thermodiffusion are described. The computational implementation of the algorithms and the computational cases are discussed in Sections 2.4 and 2.5, respectively. The results and findings are discussed in Section 2.6 and the conclusions are drawn in Section 2.7. 


\subsection{Fundamental of Thermodiffusion Phenomenon}

The mathematical modeling of thermodiffusion phenomenon can be described via the theory of linear non-equilibrium of irreversible thermodynamics (LNET) theory that associates non-equilibrium flow quantities like internal energy, heat and mass fluxes with thermodynamic forces including temperature and components' chemical potential gradients via phenomenological coefficients [120]. Specifically, the following formulations represent governing equations of LNET theory for a binary mixture in the absence of viscous dissipation and chemical reaction as well as external forces [120]:

$\vec{J} q=-L_{q q} \frac{\vec{\nabla} T}{T^{2}}-L_{q 1} \frac{\vec{\nabla}_{T}\left(\mu_{1}-\mu_{2}\right)}{T^{2}}$

$\vec{J}_{1}=-L_{1 q} \frac{\vec{\nabla} T}{T^{2}}-L_{11} \frac{\vec{\nabla}\left(\mu_{1}-\mu_{2}\right)}{T^{2}}$

In the above equations, $J_{q}$ and $J_{l}$ represent internal energy flux $\left(\mathrm{J} \cdot \mathrm{m}^{-2} \cdot \mathrm{s}^{-1}\right)$ and mole flow rate in the mixture mole $\left(\mathrm{mol} \cdot \mathrm{m}^{-2} \cdot \mathrm{s}^{-1}\right)$, respectively. $T$ is temperature $(\mathrm{K})$ and $\mu$ is chemical potential $\left(\mathrm{J} \cdot \mathrm{mole}^{-1}\right)$. The subscripts $l$ and $q$ denote component 1 and heat transfer, respectively. $L_{i j}$ terms in the two equations are the Onsager phenomenological coefficients. They associate the flow quantities like internal energy flux and mole flow rate with thermodynamic forces like temperature and chemical potential gradient through a linear function [120].

On the other hand, the mole flow rate of component 1 can also be written through conventional transport equation as [121]:

$\vec{J}_{1}=-C\left[D \vec{\nabla} x_{1}+D_{T} \vec{\nabla} T\right]$ 
where, $C, D$, and $D_{T}$ denote molar concentration (mole. $\mathrm{m}^{-3}$ ), molecular diffusion coefficient $\left(\mathrm{m}^{2} \cdot \mathrm{s}^{-1}\right)$, and Thermodiffusion coefficients $\left(\mathrm{m}^{2} \cdot \mathrm{s}^{-1} \cdot \mathrm{K}^{-1}\right)$, respectively. $x_{l}$ is mole fraction of component 1 .

At steady state, when the net flux is zero, a comparison of the above equations will yield an expression for the thermodiffusion factor $\left(\alpha_{T}\right)$ as:

$$
\alpha_{T}=T \frac{D_{T}}{D}=-\frac{T}{x_{1}\left(1-x_{1}\right)}\left(\frac{\vec{\nabla} x_{1}}{\vec{\nabla} T}\right)_{\vec{j}_{1}=0}
$$

In a multiscale modelling of heat and diffusion processes within a macroscale system such as an oil reservoir, it is critical to obtain these coefficients at the microscopic locations. While experiments are often conducted to estimate these coefficients, an alternative approach has been to employ the principles of molecular dynamics to derive these coefficients or a relation between them $\left(\alpha_{T}\right)$. As mentioned in the Introduction section, in this study, after evaluating the RNEMD and HEX algorithm-based MD approaches with respect to two binary systems, we propose a modified version of the HEX algorithm to minimize the computing time as well as overcome the instability problems faced by HEX algorithm for small systems.

\subsection{Details of MD Techniques}

\subsubsection{Heat Generation Methods}

For this study, we have developed in-house MD code based on HEX [73] and RNEMD model [75]. The overview of the principles and underlying equations in MD is described in Appendix A. Additionally, a modified heat exchange algorithm is also proposed to enhance the computing speeds and mitigate the occasional instabilities in the investigations of small systems. It must be noted that in the literature, systems with more than 1000 particles are considered as a large system [86]. As an underlying principle, in all three non-equilibrium MD formulations, heat flux and the consequent temperature 
gradient are introduced via rescaling particles' velocities at certain locations inside the system. As a result, linear distributions of temperature and concentration will be introduced to the system. At a quasi-steady state, the thermodiffusion factor can be computed directly based on the ratio of the slope of these aforementioned distributions using equation (2.4).

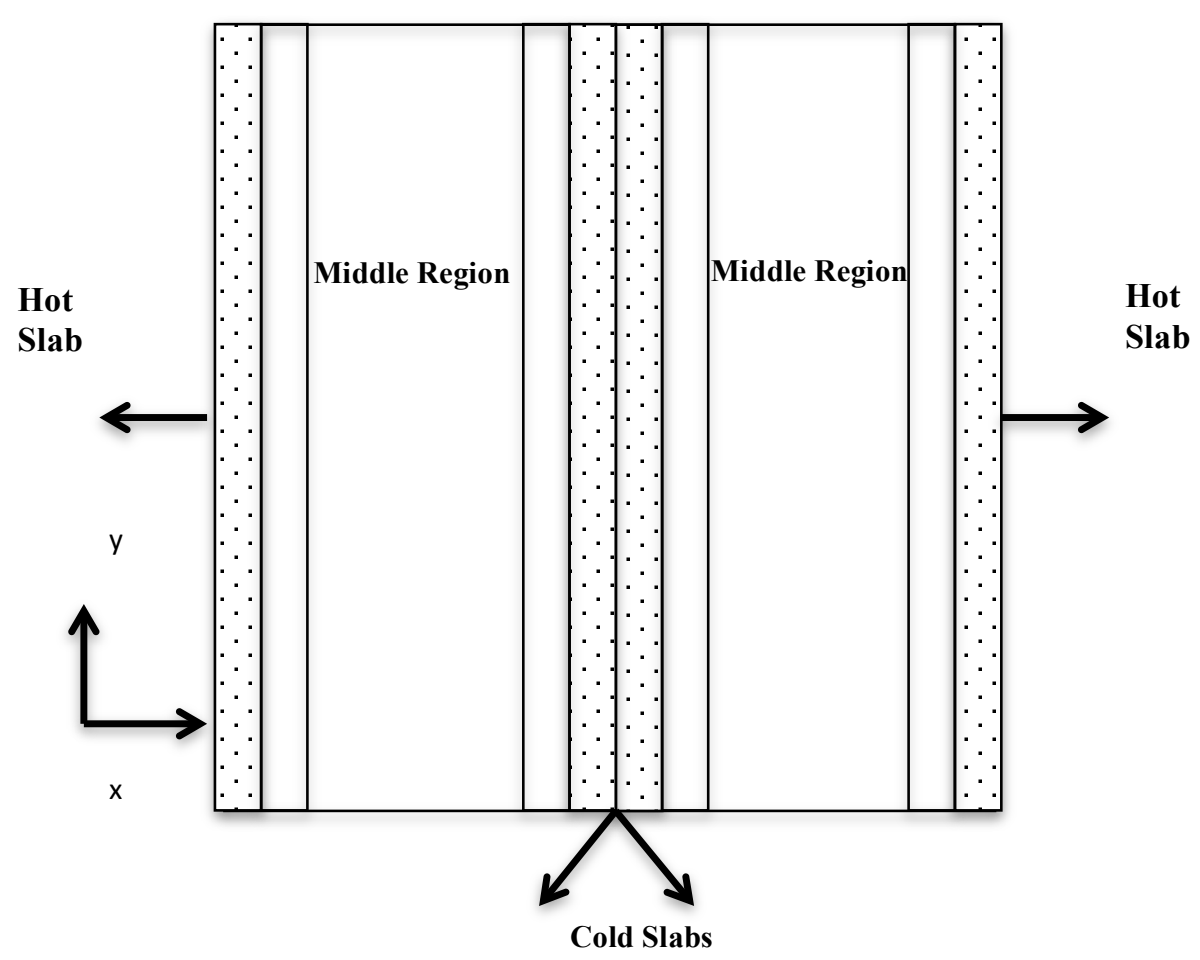

Fig. 2. 1: Schematic view of simulation box [78].

In the computational implementation of this strategy, the simulation domain is divided into three main zones: namely, hot, middle and cold regions (c.f. Figure 2.1). In RNEMD method, the velocities of identical components in cold and hot layers are swapped at fixed time intervals to generate the heat flow in the system [75]. As will be shown later, this swapping interval has a significant influence on the end result. On the other hand, in the HEX algorithm, heat flux is introduced by adding certain amount of energy to hot zone and simultaneously extracting the same amount from cold region without violating the law of conservation of momentum. In the implementation of HEX algorithm, two 
quadratic equations that must be solved to update the velocities in every time step [73] are:

$$
\begin{aligned}
& \Delta \vec{U}_{h}=\frac{1}{2} \sum_{k_{h}=1}^{N_{h}} m_{k_{h}}\left[\left(\left(1+\gamma_{h}\right) \vec{V}_{k_{h}}-\gamma_{h} \vec{V}_{b_{h}}\right)^{2}-\vec{V}_{k_{h}}^{2}\right] \\
& \Delta \vec{U}_{c}=\frac{1}{2} \sum_{k_{c}=1}^{N_{c}} m_{k_{c}}\left[\left(\left(1+\gamma_{c}\right) \vec{V}_{k_{c}}-\gamma_{c} \vec{V}_{b_{c}}\right)^{2}-\vec{V}_{k_{c}}^{2}\right]
\end{aligned}
$$

where, $N, \Delta U$ and $V_{b}$ represent number of particles in the hot (h) or cold (c) layers, the amount of energy $(\mathrm{J})$ and barycentric velocity $\left(\mathrm{m} . \mathrm{s}^{-1}\right)$ of particles in the region, respectively. Also, $m_{k}$ and $V_{K}$ are the mass $(\mathrm{kg})$ and velocity $\left(\mathrm{m} \cdot \mathrm{s}^{-1}\right)$ of the $\mathrm{k}^{\text {th }}$ particle, respectively. $\gamma$ is a scaling factor, and the terms in right hand sides' parentheses are the rescaled velocities. It must be noted that in order to have real roots, the discriminants of above equations must be greater than zero.

\section{Modified HEX algorithm:}

While swapping interval in the RNEMD plays a key role in the equilibrium solution, with HEX algorithm, in small to medium size systems, occasionally the algorithms can fail to meet the required criteria to obtain real solutions for aforementioned quadratic formula, equations (2.5) and equations (2.6). Consequently, this can cause some inaccurate unphysical disturbances in generation of heat flow into the system that can lead to incorrect solutions.

To overcome these drawbacks, we propose a modification of the HEX algorithm in which the particles' velocities are upgraded by constant factors $(\zeta)$ in hot and cold slabs. Specifically, the following equation is used to rescale the velocity:

$\vec{V}_{i}=\vec{V}_{i}^{\prime}(1 \pm \zeta) \mp \zeta \vec{V}_{b}$ 
where, $V_{i}$ and $V_{i}$ are the velocity before and after rescaling in time step, respectively. From experience we know the typical fluctuations of $\gamma$ values in equation (2.5) and equation (2.6) and we have noticed the changes are in the order of $10^{-3}$. Hence, for this

study, we have set the value of $\zeta$ to 0.005 and 0.0025 for $\mathrm{Ar}-\mathrm{Kr}$ system and hydrocarbon mixtures, respectively. This modification eliminates the iterative process needed to calculate the roots of equations (2.5) and (2.6) completely, thereby directly contributing in the reduction of the computational time.

\subsubsection{Pair Potential Functions and General MD Parameters}

To describe the interaction potential, we have chosen the simple Lennard-Jones (LJ) potential with a cut-off distance of $r_{c}=2.5 \sigma_{i j}$ for all boundary-driven methods [122]. We would like to add that this simple LJ potential has been successfully used in the literature for hydrocarbon mixtures $[84,86]$. Additionally, it has also been shown in the literature that this simple LJ method has a better quantitative agreement with experimental data in comparison to more complicated LJ methods [86]. The mathematical representation of the LJ potential is:

$\phi\left(r_{i j}\right)=4 \varepsilon_{i j}\left[\left(\frac{\sigma_{i j}}{r_{i j}}\right)^{12}-\left(\frac{\sigma_{i j}}{r_{i j}}\right)^{6}\right]$

where, $\phi, \varepsilon_{i j}, \sigma_{i j}$ and $r_{i j}$ are pair potential (J), well-depth potential (J), atomic diameter (m), and distance between particles (m). subscripts $i$ and $j$ denote dissimilar particles.

In order to obtain the potential parameter for dissimilar particles, the following LorentzBerthelot mixing rules have been applied [122]:

$\sigma_{i j}=0.5\left(\sigma_{i i}+\sigma_{i j}\right)$ 
$\varepsilon_{i j}=\sqrt{\varepsilon_{i i} \varepsilon_{j j}}$

The LJ parameters of identical particles that will be needed in the above equations are summarized in Table 2.1.

Table 2.1: Lennard-Jones potential parameters. These parameters were obtained from NIST Thermophysical Properties of Hydrocarbon Mixtures Database [123].

\begin{tabular}{|c|c|c|c|c|c|}
\hline Material & $\mathbf{A r}$ & $\mathbf{K r}$ & $\mathbf{C}_{\mathbf{5}}$ & $\mathbf{C}_{\mathbf{6}}$ & $\mathbf{C}_{\mathbf{1 0}}$ \\
\hline$\varepsilon / \mathrm{k}_{\mathrm{b}}(\mathrm{K})$ & 119.8 & 167 & 346 & 393 & 471 \\
\hline$\sigma(\mathrm{nm})$ & 0.341 & 0.363 & 0.545 & 0.595 & 0.68 \\
\hline
\end{tabular}

\subsection{Computational Implementation}

For the computational domain, a cubic volume assimilated using 32 slabs of identical thickness in the direction of the desired heat flux, i.e. x direction, has been used. The front view of the three-dimensional domain, as seen from the z-axis is shown in Fig. 2.1. The two layers at ends of our simulation box are the hot zones whereas the two middle slabs are cold regions in the schematic represented in Fig. 2.1.

For each particle, knowing the total potential, the negative gradient of this potential is the force experienced by this particle. By applying the Newton's law to each particle, we can calculate the particle's acceleration. Subsequently, by employing the Verlet velocity integration method we can calculate the velocity and position of each particle at every time step [95].

In all the MD simulations, the particles were initially randomly positioned inside the simulation box, whereas the initial velocities of the particles were characterized via Maxwell-Boltzmann distribution function at 30\% below the desired temperature. Then we let the system reach the equilibration period through rescaling velocity to the desired temperature. The equilibration period took about 200,000 iterations. Subsequently, using this equilibrated state as the starting point of the thermodiffusion simulations, heat flux 
was introduced to the system, and the simulations were continued for an additional $1,000,000$ iterations. In all simulations, periodic boundary conditions were applied across all three directions of the simulations box and the minimum image convention was used to reduce the wall impacts. Also, in all the simulations, the Gaussian (velocity-rescaling) thermostat to control the system's temperature has been employed [122].

\subsection{Computational Cases}

MD simulations using all three algorithms, namely, RNEMD, HEX and mHEX, have been made for equimolar mixtures of $\mathrm{Ar}-\mathrm{Kr}$, and a mixture of $\mathrm{nC}_{6}-\mathrm{nC}_{10}$ in which the mole fraction of $\mathrm{nC}_{6}=0.62$. The choice of an equimolar mixture of $\mathrm{Ar}-\mathrm{Kr}$ is because of ample data in the literature for this composition. The composition for $\mathrm{nC}_{6}-\mathrm{nC}_{10}$ was chosen because of the availability of experimental data. Additionally, we have examined the performance of the HEX and modified HEX method for three different states of nonequimolar $\mathrm{nC}_{5}-\mathrm{nC}_{10}$ mixture that have been studied in the literature via $\mathrm{MD}$ simulations as well as experimental technique.

In order to decrease the statistical uncertainty due to random nature of the MD techniques, the simulation for each individual mixture was repeated 4 times. The dimensionless time step for the hydrocarbon mixtures was 0.0008 and each run consisted of more than 1,000,000 iterations. On the other hand, the dimensionless time step for ArKr mixture was 0.002. Additionally, in all runs, the system's temperature and density of the mixture were kept fixed. The number of particles for the Ar-Kr mixture was 500, whereas for the hydrocarbon mixtures, 1000 particles were included in the system. The value of the reduced heat flux $\left(J_{u}{ }^{*}\right)$ was 0.5 for all mixtures.

\subsection{Results and Discussions}

In this section, we present the results from the simulation of the three binary systems using the MD algorithms. In presenting the results, comparisons have been made with the experimental as well as molecular dynamics data available in the literature. 


\subsection{1 $\mathrm{Ar}-\mathrm{Kr}$}

In this subsection, the thermodiffusive separation of mixture of $\mathrm{Ar}-\mathrm{Kr}$ at its liquid state above the triple point using the three MD techniques is presented. The specific thermodynamic sate of this mixture that is considered in this simulation is the one investigated by other researchers in the literature [37- 38, 40, 55] and corresponds to dimensionless temperature $T^{*}=\frac{T}{\varepsilon_{11} / k_{b}}=0.9650$ and dimensionless density, $\rho^{*}=\frac{N \sigma_{11}}{V}$ $=0.7137$.

For this equimolar system, the average temperature distribution inside the domain at steady state as calculated by the RNEMD, HEX and mHEX algorithm is shown in Fig. 2.2. As seen in this figure, there is a good agreement in the profile of the temperature in the middle region of the domain. However, closer to the hot slabs and the cold slabs, there is a disagreement in the temperature values. This is expected because the algorithms introduce heat flux into the system by affecting the values of velocities in these end slabs. Given that each algorithm does this differently, there is a disagreement in the temperature values at these zones. In fact, given that in RNEMD this is done by directly swapping the velocities, this algorithm has strong spikes in temperature at the hot and cold slabs (c.f. Fig. 2.2).

The concentration profile of $\mathrm{Kr}$ and $\mathrm{Ar}$ in the middle region in Fig. 2.1 are shown in Fig. 2.3 and Fig. 2.4, respectively. As seen in these figures, Kr moves towards the cold region (higher concentration near the cold region) and Ar moves towards the hot region (higher concentration near the hot region). Further, the prediction from all three methods is in close agreement with each other. 


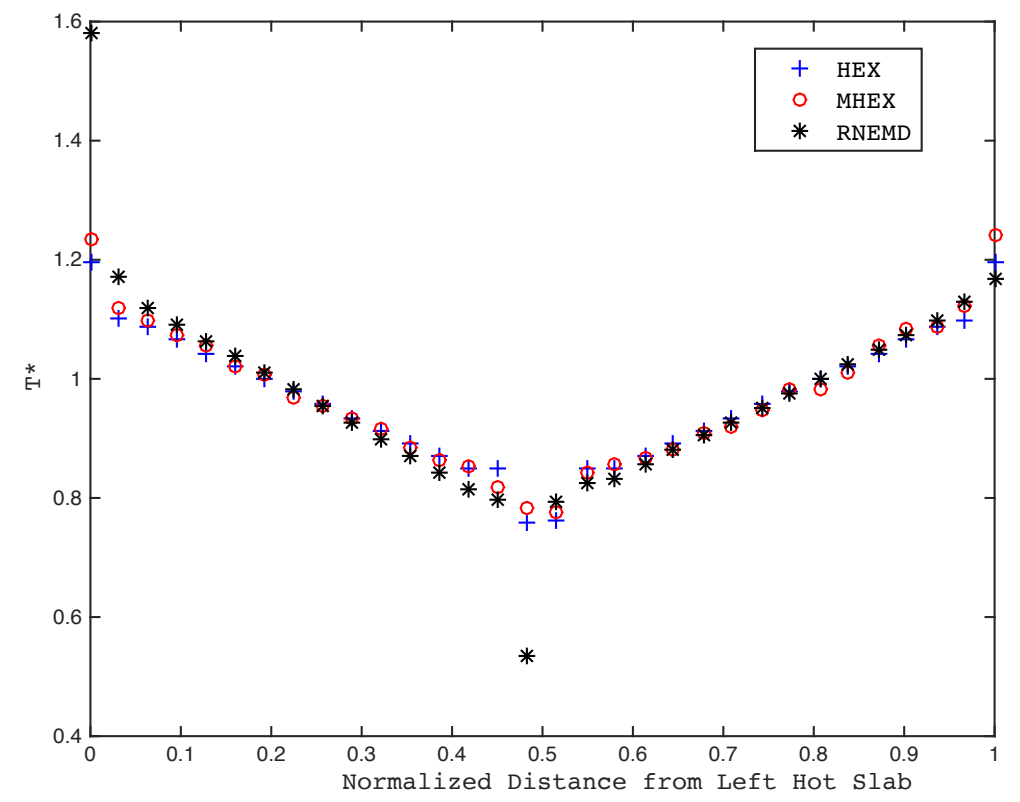

Fig. 2.2: Dimensionless temperature distribution inside the simulation box for equimolar mixture of Ar-Kr using the HEX, RNEMD (with swapping time $=20$ time step) and mHEX algorithms [78].

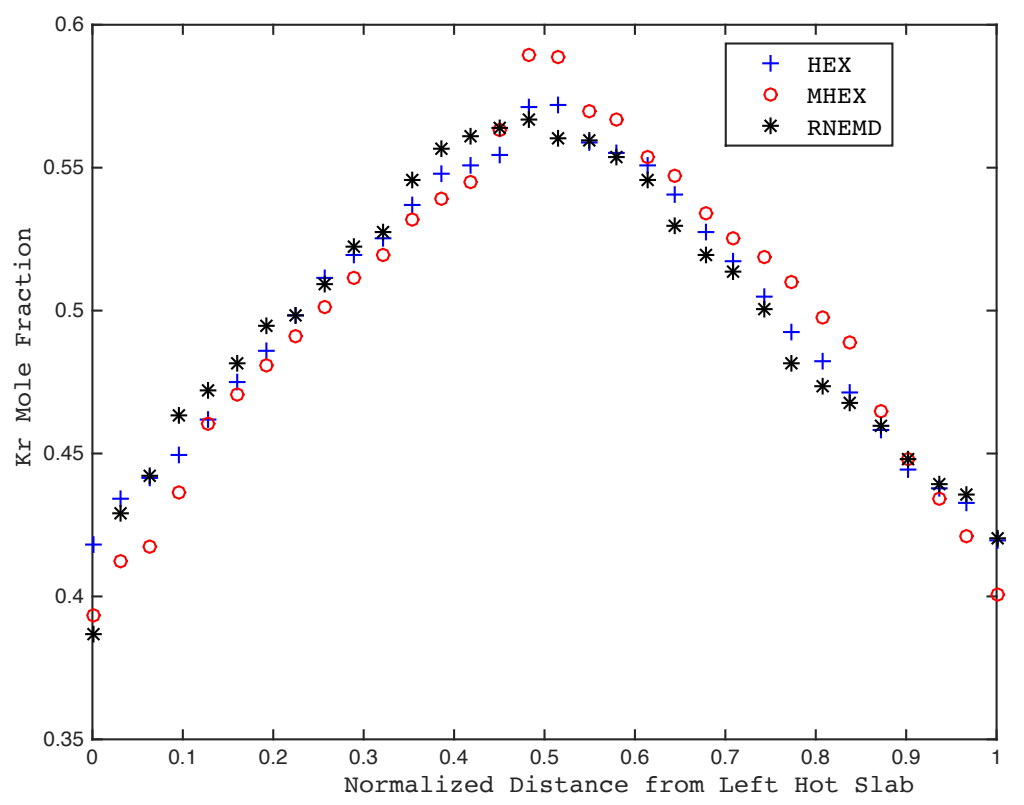

Fig. 2.3: $\mathrm{Kr}$ concentration profile inside the simulation box for equimolar mixture of $\mathrm{Ar}-\mathrm{Kr}$ using the HEX, RNEMD (with swapping time =20 time step) and MHEX algorithms [78]. 
The calculated values of $\alpha_{\mathrm{T}}$ are summarized in Table 2.2 along with the values from the literature. As seen in this table, the data from this work and the literature indicates that the value of $\alpha_{\mathrm{T}}$ varies between 1.6 and 2.4 wherein our MD results for algorithm heat generations overlap with the findings in the literature. However, since the details and procedures of MD simulations differ including the integration method, pair potential functions, and cut off ratio the comparison between them must be done with cautious.

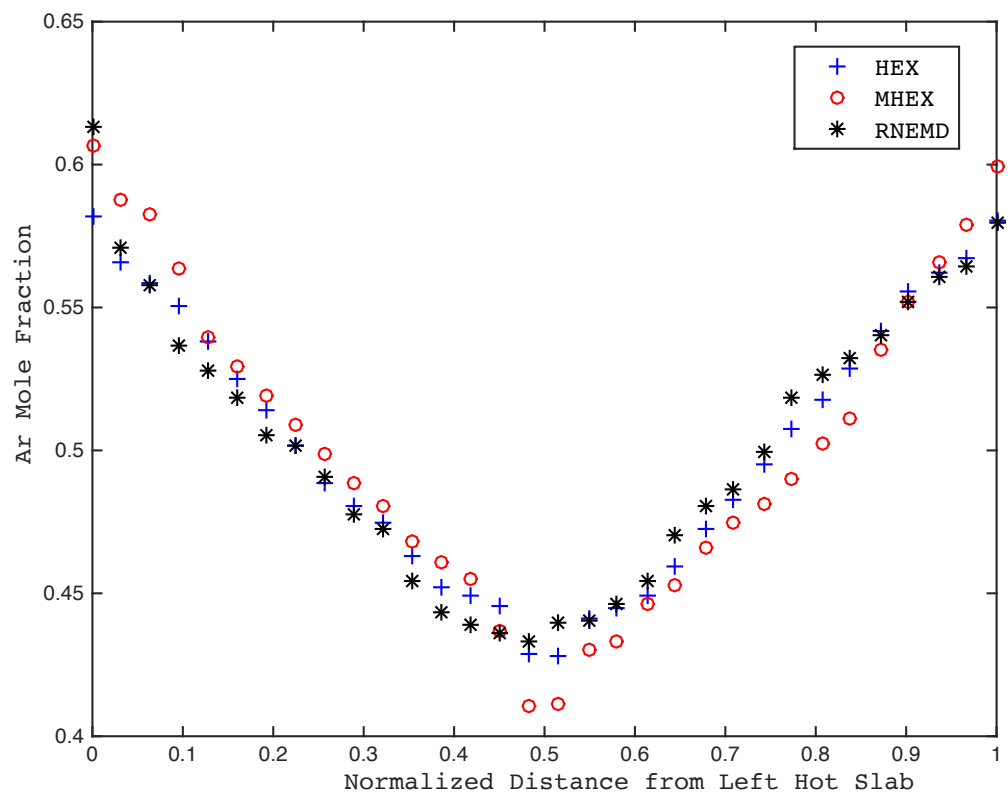

Fig. 2.4: Ar concentration profile inside the simulation box for equimolar mixture of Ar-Kr using the HEX, RNEMD (with swapping time $=20$ time step) and MHEX algorithms [78].

Table 2.2: Predicted thermodiffusion factor for equimolar mixture of Ar-Kr for HEX, mHEX and RNEMD method with swapping time $=20$ iteration $[78]^{\mathrm{a}}$.

\begin{tabular}{|c|c|}
\hline Reference & $\boldsymbol{\alpha}_{\mathbf{T}}$ \\
\hline MD, SNEMD. [70] & $1.6 \pm 0.5$ \\
\hline MD, SNEMD. [115] & $2.4 \pm 0.4$ \\
\hline MD, HEX. [73] & $1.78 \pm 0.07$ \\
\hline MD, GK-EMD [124] & $1.6 \pm 0.1$ \\
\hline HEX [78] & $2.02 \pm 0.12$ \\
\hline mHEX [78] & $1.91 \pm 0.13$ \\
\hline RNEMD [78] & $1.58 \pm 0.27$ \\
\hline
\end{tabular}

${ }^{a}$ All error bars related to repeatability errors for MD approaches. 


\subsection{2 $n C_{6}-n C_{10}$}

As in the Ar-Kr system, a similar analysis was done for the n-hexane - n-decane system. Specifically, the computational results from the MD simulations were compared with experimental data available for non-equimolar system of $\mathrm{nC}_{6}-\mathrm{nC}_{10}$ at $T^{*}=0.6123$ and $\rho^{*}=1.2130$ with mole fraction of $\mathrm{nC}_{6}$ as 0.62 . The average temperature distribution inside the domain at steady state as calculated by the RNEMD, HEX and mHEX algorithm is shown in Fig. 2.5. Due to symmetrical pattern observed inside the total domain, the average temperature in just the first half of the domain is plotted. As seen in this graph, all three methods are in close agreement with each other with the RNEMD method converging to slightly higher temperatures (about 4-5\%) than the HEX and mHEX algorithms. Further, in all three methods, is the deviation in the temperature distribution is about $\pm 0.008 \%$.

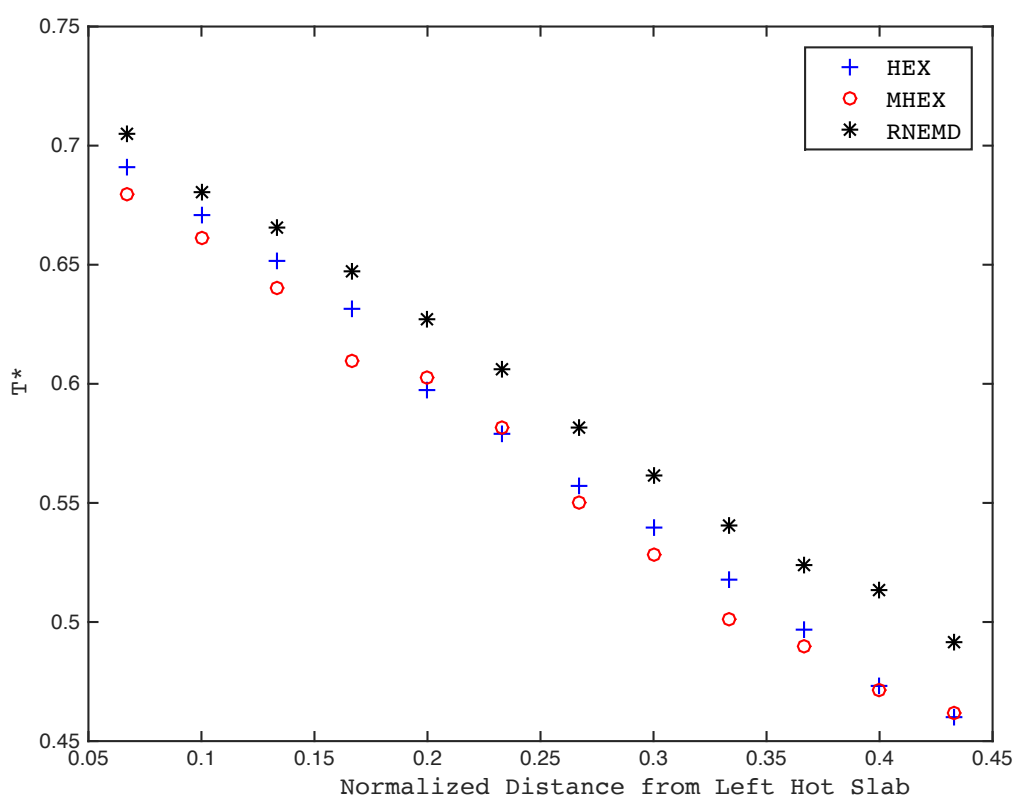

Fig. 2.5: Average dimensionless temperature distribution in middle layers for non-equimolar $\mathbf{n C}_{6^{-}}$ $\mathrm{nC}_{10}$ mixture using the HEX, RNEMD (with swapping time $=20$ time step) and MHEX algorithms [78].

The concentration profile of $\mathrm{nC}_{10}$ and $\mathrm{nC}_{6}$ in the middle zone in Fig. 2.1 are shown in Fig. 2.6 and Fig. 2.7, respectively. As seen in these graphs, $\mathrm{nC}_{10}$ accumulates near the cold zone while lighter component moves towards the hot zone. Moreover, the rates of 
change in concentration profile, i.e. the concentration gradient, for $\mathrm{nC}_{10}$ and $\mathrm{nC}_{6}$ are nearly the same for all three algorithms. It must be noted that that $y$-axis range in these figures are quite small for clarity in the representation of the data.

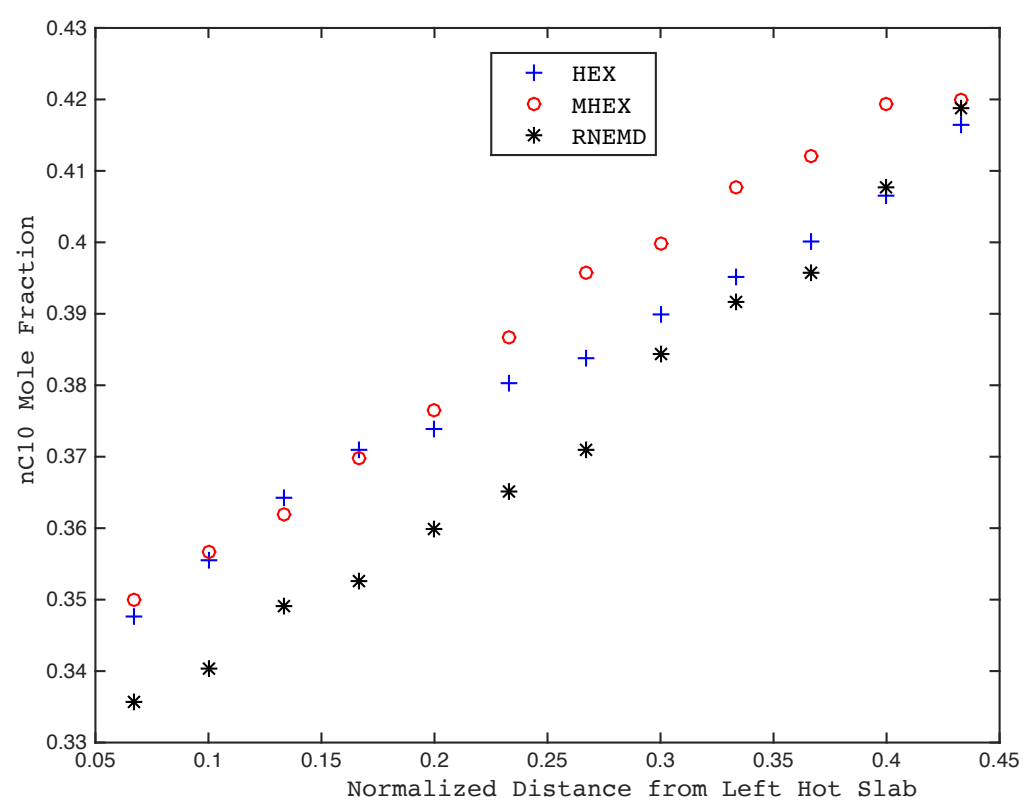

Fig. 2.6: Average mole fraction trend of $\mathrm{nC}_{10}$ in middle layers for non-equimolar $\mathrm{nC}_{6}-\mathrm{nC}_{10}$ mixture using the HEX, RNEMD (with swapping time $=20$ time step) and $\mathbf{m H E X}$ algorithms [78].

The predicted values of the Thermodiffusion factor, $\alpha_{T}$, are summarized in Table 2.3 along with the experimental value from the literature. As seen in this table, the value of thermodiffusion factor varies between 0.67 and 0.96. Comparing the results of the three molecular dynamics simulations with respect to the experimental data, we find that as in the Ar-Kr mixture, the RNEMD method has the largest relative error of $21.52 \%$ with respect to the experimental data, predicting the thermodiffusion factor of approximately $0.96 \pm 0.16$. The HEX algorithm is marginally more accurate than RNEMD and predicts thermodiffusion factor close $0.67 \pm 0.13$ with a $15.19 \%$ relative error with respect to the experimental data. Finally, the modified HEX method is the most accurate, predicting the thermodiffusion factor as $0.83 \pm 0.17$. This is a relative error of $5.63 \%$ with respect to the experimental data. Thus, once again the newly proposed method is more accurate than the HEX and RNEMD algorithms. 


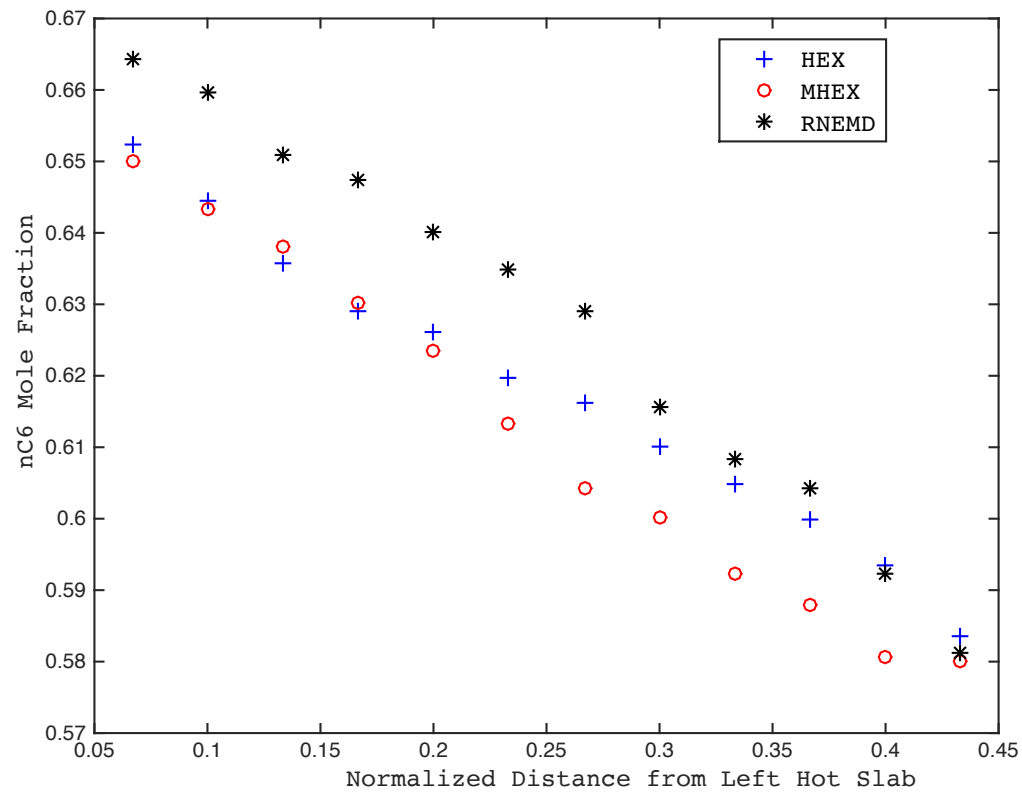

Fig. 2.7: Average mole fraction trend of $\mathrm{nC}_{6}$ in middle layers for non-equimolar $\mathrm{nC}_{6}-\mathrm{nC}_{10}$ mixture using the HEX, RNEMD (with swapping time $=20$ time step) and MHEX algorithms [78].

Table 2.3: Predicted thermodiffusion factor for equimolar mixture of $\mathrm{nC}_{6}-\mathrm{nC}_{10}$ for $\mathrm{HEX}$, MHEX and RNEMD method with swapping time $=20$ iteration $[78]^{\mathrm{a}}$.

\begin{tabular}{|c|c|c|}
\hline Reference & $\boldsymbol{\alpha}_{\mathbf{T}}$ & Relative Error with respect to experiment (\%) \\
\hline Expt. [125] & $0.79 \pm 0.04$ & - \\
\hline HEX this work & $0.67 \pm 0.13$ & 15.19 \\
\hline MHEX this work & $0.83 \pm 0.17$ & 5.63 \\
\hline RNEMD this work & $0.96 \pm 0.16$ & 21.52 \\
\hline
\end{tabular}

All error bars related to repeatability errors for experimental and MD approaches. The thermogravitational column technique was used in reference [125] .

\subsection{3 $n C_{5}-n C_{10}$}

Finally, to increase our confidence in the MHEX algorithm, a third mixture of $\mathrm{nC}_{5}-\mathrm{nC}_{10}$ at three different mole fractions of pentane, namely, $0.2,0.5$ and 0.8 , respectively was studied. These compositions correspond to three different states, i.e., $\rho^{*}=1.0491$, $\rho^{*}=1.2088$, and $\rho^{*}=1.4212$, all with a dimensionless temperature of $T^{*}=0.6363$. Knowing that the RNEMD method is expected to have large errors, the focus was to employ only the HEX and MHEX algorithms to study these mixtures. 


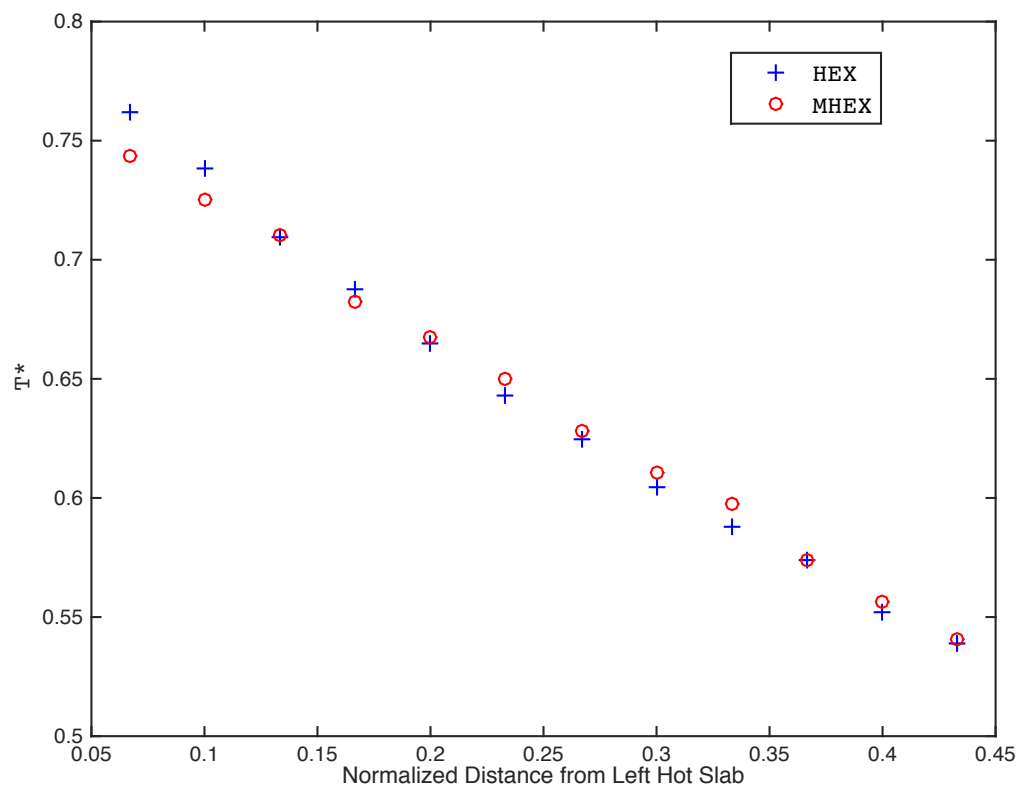

Fig. 2. 8: Average dimensionless temperature distribution in middle layers for $\mathrm{nC}_{5}-\mathrm{nC}_{10}$ mixture with an initial uniform mole fraction of $\mathrm{nC}_{5}=0.8$, using the $\mathrm{HEX}$, and $\mathrm{MHEX}$ algorithms [78].

The average temperature distribution inside the domain at steady state for HEX, and MHEX for the mixture with the mole fraction of $\mathrm{nC}_{5}$ at 0.8 is shown in Fig. 2.8. The concentration profiles of $\mathrm{nC}_{10}$ and $\mathrm{nC}_{5}$ for this mixture in the middle zone in Fig. 2.1 are shown in Fig. 2.9 and Fig. 2.10, respectively. It is evident that as in the previous mixtures, the heavy component, i.e., $\mathrm{nC}_{10}$, moves toward the cold zone, whereas $\mathrm{nC}_{5}$ accumulates near the hot zone. 


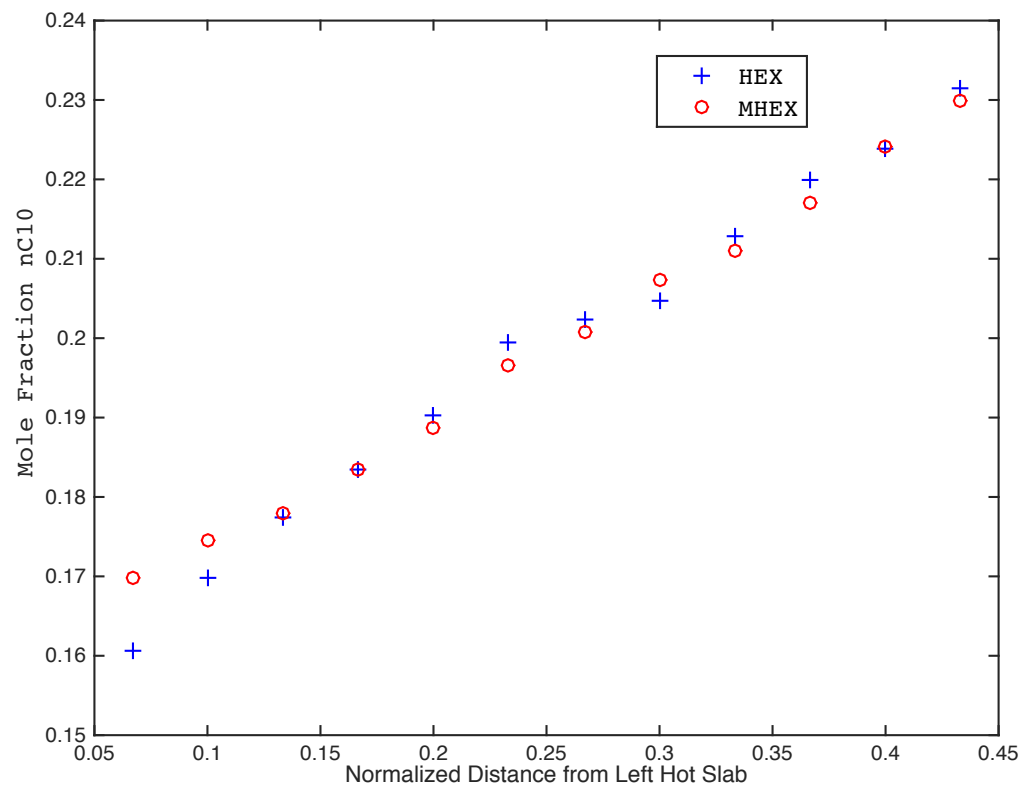

Fig. 2.9: Average mole fraction trend of $\mathrm{nC}_{10}$ in middle layers for $\mathrm{nC}_{5}-\mathrm{nC}_{10}$ mixture with an initial uniform mole fraction of $\mathrm{nC}_{5}=0.8$, using the $\mathrm{HEX}$, and $\mathrm{MHEX}$ algorithms [78].

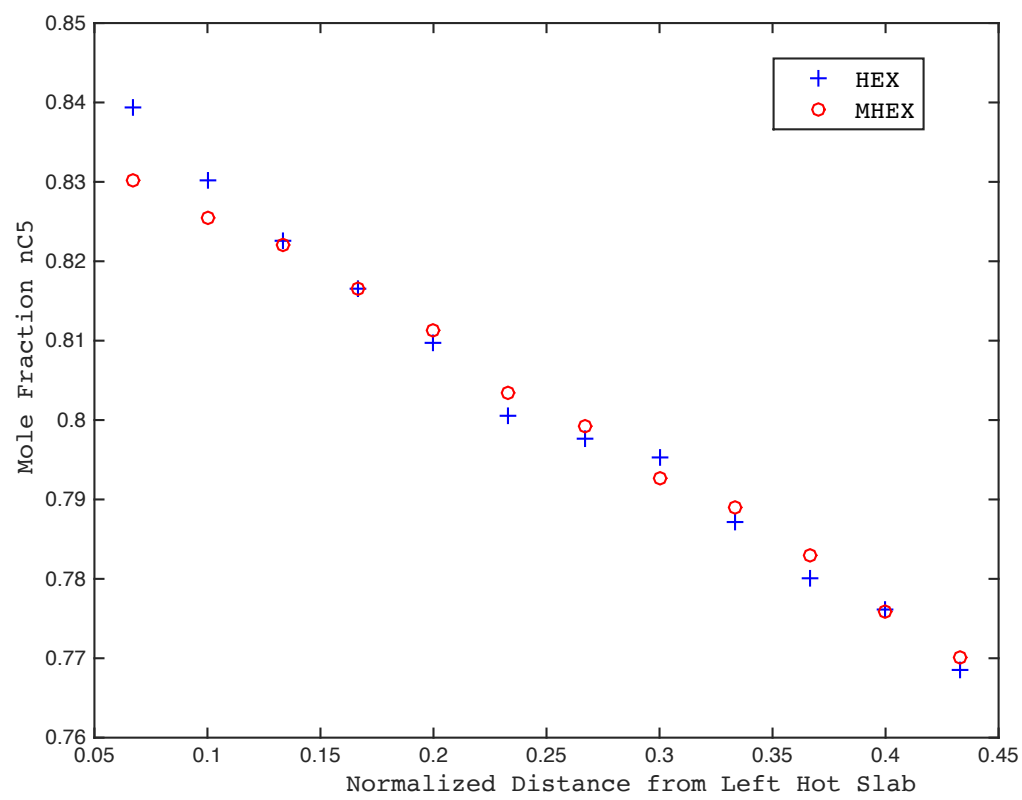

Fig. 2.10: Average mole fraction trend of $\mathrm{nC}_{5}$ in middle layers for $\mathrm{nC}_{5}-\mathrm{nC}_{10}$ mixture with an initial uniform mole fraction of $\mathrm{nC}_{5}=0.8$, using the $\mathrm{HEX}$, and $\mathrm{MHEX}$ algorithms [78].

The thermodiffusion factor calculated using the gradients of temperature and concentration in the middle region in equation (2.4) using either algorithm is summarized 
in Table 2.4. Additionally, experimental data as well as the values of Thermodiffusion factor from the other MD studies are also included in this table. As summarized in the table, the results from the present study show a good agreement with experimental data. Specifically, in all three mixtures, the mHEX algorithm performed better than the HEX algorithm in predicting the Thermodiffusion factor. More precisely, the accuracy in predicting the Thermodiffusion coefficient was between approximately 3\% and $9 \%$, depending upon the composition of the mixture. In comparing the performance of mHEX with the SNEMD data available in the literature it is found that except at the mole fraction of 0.2 for $\mathrm{nC}_{5}$, the SNEMD estimates of the Thermodiffusion factor were quite erroneous. In the anomalous case where the mole fraction of $\mathrm{nC}_{5}$ is 0.2 , SNEMD was about $6 \%$ more accurate than mHEX predictions.

Table 2.4: Predicted thermodiffusion factor for three different mixtures $\mathrm{nC}_{5}-\mathrm{nC}_{10}$ for $\mathrm{HEX}$, $\mathrm{mHEX}$. Numbers in the parentheses indicate the relative error with respect to the experimental data [78] ${ }^{\mathrm{a}}$.

\begin{tabular}{|c|c|c|c|c|}
\hline \multirow{2}{*}{ nC $_{5}$ Mole Fraction } & \multicolumn{4}{|c|}{$\boldsymbol{\alpha}_{\mathbf{T}}$} \\
\cline { 2 - 5 } & \multirow{2}{*}{ Exp. [126] } & \multicolumn{2}{|c|}{ This Work } & Literature \\
\cline { 3 - 5 } & & mHEX & HEX & SNEMD. [126] \\
\hline \multirow{2}{*}{0.8} & \multirow{2}{*}{$1.06 \pm 0.25$} & $1.18 \pm 0.25$ & $1.21 \pm 0.12$ & $1.34 \pm 0.25$ \\
& & $(10.17 \%)$ & $(14.15 \%)$ & $(20.89 \%)$ \\
\hline \multirow{2}{*}{0.5} & \multirow{2}{*}{$0.98 \pm 0.23$} & $0.95 \pm 0.12$ & $0.92 \pm 0.15$ & $1.08 \pm 0.83$ \\
& & $(3.16 \%)$ & $(6.12 \%)$ & $(10.20 \%)$ \\
\hline \multirow{2}{*}{0.2} & \multirow{2}{*}{$1.14 \pm 0.27$} & $0.98 \pm 0.17$ & $0.88 \pm 0.20$ & $1.05 \pm 0.57$ \\
& & $(14.04 \%)$ & $(22.81 \%)$ & $(7.89 \%)$ \\
\hline
\end{tabular}

${ }^{a}$ All error bars related to repeatability errors for experimental and MD approaches. The experimental technique used in reference [126] was thermal diffusion forced Rayleigh scattering (TDFRS).

From the results of all the mixtures analyzed so far, it can be argued that given that the mHEX algorithm consistently performs better than the original HEX algorithm for the mixtures investigated in this study, this modified algorithm is a good candidate to be employed to study Thermodiffusion in liquid mixtures. 


\subsubsection{Effect of Swapping Time in RNEMD}

It is evident from the thermodiffusion values in Tables 2.2 and 2.3 that the RNEMD is able to predict the thermodiffusive separation only qualitatively and that the values of Thermodiffusion factor are much further from the experimental or the average values reported in the literature. The relatively large quantitative discrepancy in RNEMD method and its modified variants with respect to the experimental data on thermodiffusion has also been reported in the literature [76, 77]. As mentioned earlier, this is most likely attributed to the swapping interval, when one abruptly interchanges the velocities of particles in the cold and the hot zone, leading to an unnatural disturbance in the system. To investigate this further, we conducted MD simulations of all two Ar-Kr and $\mathrm{nC}_{6}-\mathrm{nC}_{10}$ mixtures using $\mathrm{RNEMD}$ algorithm and studied three different swapping intervals, i.e., 20, 40 and 80 time steps.

The thermodiffusion factors from all these simulations are plotted in Fig. 2.11. As seen in this figure, in two mixtures, there is a significant variation in the values of thermodiffusion factor as we increase the swapping interval. Interestingly, both mixtures exhibit a somewhat quadratic behaviour in the values of $\alpha_{T}$. These large variations clearly indicate that one must exercise caution in studying the values $\alpha_{\mathrm{T}}$ of from this algorithm.

\subsubsection{Computational Time}

In studying systems using molecular dynamics techniques, computational time is an important factor that often determines the permissible size of the system. Table 2.5 shows the average computational time per 1000 iterations for three different systems. For the Ar-Kr system, the computational domain had 500 particles, whereas the hydrocarbon systems had a computational domain with 1000 particles. Further, for the RNEMD simulations, a swapping interval of 20 was used. 


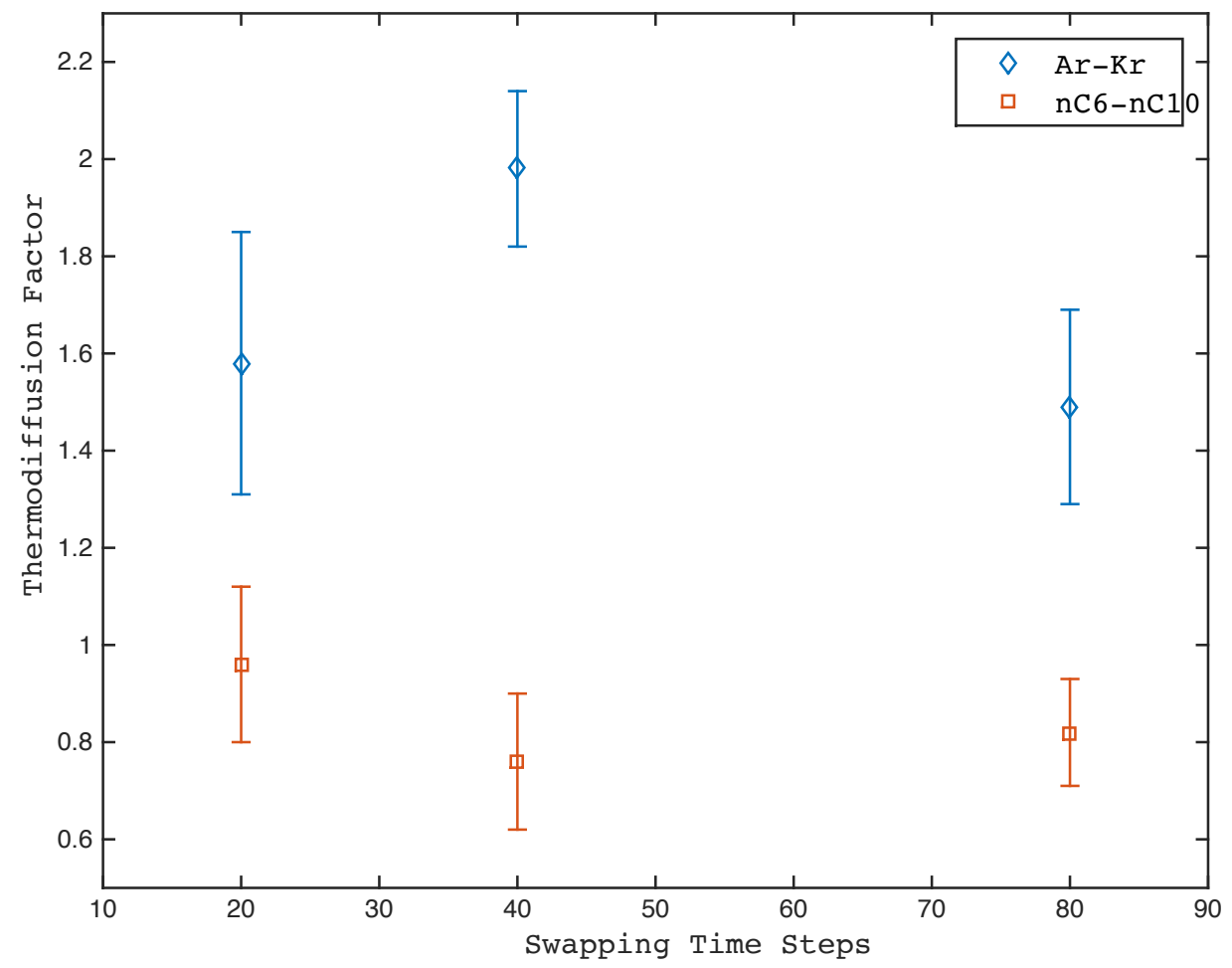

Fig. 2.11: Thermodiffusion factor versus velocity swapping time for RNEMD method [78].

As seen in this table, the HEX method is generally the slowest algorithm in all three systems. On the other hand, for the RNEMD system, had a moderate computational speed. The mHEX method requires the least computation time, i.e., 17.1293sec, and $36.5197 \mathrm{sec}$, for the $\mathrm{Ar}-\mathrm{Kr}$ and $\mathrm{nC}_{6}-\mathrm{nC}_{10}$, respectively. Also, for the three $\mathrm{nC}_{5}-\mathrm{nC}_{10}$ mixtures with different mole fractions, $\mathrm{mHEX}$ required lower computational time than the HEX algorithm, i.e., 34.4519sec, 38.1569sec and 39.1747sec, when mole fraction of $\mathrm{nC}_{5}$ is equal to $0.2,0.5$ and 0.8 , respectively. More precisely, with respect to the HEX algorithm, this is a speedup of slightly over $7 \%$. Knowing that the computational time does not scale linearly with the size of the system, assuming even a modest $7 \%$ savings in computational time can be enormous for systems with several thousands of particles that have to be simulated for a few million iterations. Put differently, in view of our long-term objective of integrating a MD simulation tool in a multi-scale framework to study a macroscale system such as an entire reservoir, the proposed mHEX algorithm is perhaps an ideal candidate. 
Table 2.5: Computational time of per 1000 iterations for HEX, mHEX and RNEMD method with swapping time $=\mathbf{2 0}$ iteration [78].

\begin{tabular}{|c|c|c|c|c|}
\hline Mixture & mHEX (sec) & HEX (sec) & $\begin{array}{c}\text { RNEMD } \\
\text { (sec) }\end{array}$ & $\begin{array}{c}\text { Time Saving } \\
\text { (HEX vs. } \\
\text { mHEX)\% }\end{array}$ \\
\hline $\mathrm{Ar}-\mathrm{Kr}$ & 17.1293 & 18.4637 & 17.5570 & $7.12 \%$ \\
\hline $\mathrm{nC}_{6}-\mathrm{nC}_{10}$ & 36.5197 & 39.3129 & 37.5755 & $6.92 \%$ \\
\hline $\mathrm{nC}_{5}-\mathrm{nC}_{10}\left(\mathrm{x}_{\mathrm{nC} 5}=0.8\right)$ & 34.4519 & 37.5750 & - & $8.21 \%$ \\
\hline $\mathrm{nC}_{5}-\mathrm{nC}_{10}\left(\mathrm{x}_{\mathrm{nC} 5}=0.5\right)$ & 38.1569 & 41.2017 & - & $7.41 \%$ \\
\hline $\mathrm{nC}_{5}-\mathrm{nC}_{10}\left(\mathrm{x}_{\mathrm{nC} 5}=0.2\right)$ & 39.1747 & 41.9639 & - & $6.64 \%$ \\
\hline
\end{tabular}

\subsection{Summary and Conclusions}

In this paper, we compared the performance of two widely used MD approaches, i.e. HEX and RNEMD, for three different mixtures, namely, an equimolar Ar-Kr mixture, a non-equimolar mixture of $\mathrm{nC}_{6}-\mathrm{nC}_{10}$ and three different mixtures of $\mathrm{nC}_{5}-\mathrm{nC}_{10}$. Comparisons were made with respect to the data from the literature (theoretical as well as experimental). The following conclusions were drawn from the findings:

(i) All three methods predict nearly the same temperature distribution in the system, for either mixture. The predictions of the RNEMD were marginally higher (4-5\%) than the other two algorithms.

(ii) The Thermodiffusion factors predicted by the RNEMD was the most erroneous with a relative error of about $22 \%$ for hydrocarbon mixture of $\mathrm{nC}_{6}-\mathrm{nC}_{10}$. This is a direct consequence of the abrupt perturbation of the system that happens with the velocity of a particle in the hot zone is swapped with a velocity of the particle in the cold zone. This disturbance in the system that might be close to equilibrium can have an unsettling effect 
on the system that can lead us to solutions further away from the experimental observations.

(iii) A closer study of the RNEMD with respect to the "swapping time", i.e., the time at which the velocities of the two particles are swapped, it was found that there were large variations in the results predicted by the MD simulations. This directly indicates that the results predicted by the RNEMD simulations must be used with greater caution. 


\section{CHAPTER 3- THERMAL DIFFUSION IN BINARY AND TERNARY HYDROCARBON MIXTURES STUDIED USING A MODIFIED HEAT EXCHANGE ALGORITHM}

This chapter is based on a published paper in the Journal of Thermal Science and Engineering Progress:

Mozaffari, S. H., Srinivasan, S. \& Saghir, M. Z.,

Thermal diffusion in binary and ternary hydrocarbon mixtures studied using a modified heat exchange algorithm,

Therm. Sci. Eng. Progress, 4, 168-174.

\subsection{Summary}

In this work, a recently proposed modified form of the heat exchange algorithm (mHEX) has been employed to conduct molecular dynamics (MD) simulations of thermodiffusion in binary and ternary hydrocarbon mixtures. Two normal alkane binary mixtures of hexane $\left(\mathrm{nC}_{6}\right)$ - docane $\left(\mathrm{nC}_{10}\right)$ and $\mathrm{nC}_{6}$-dodecane $\left(\mathrm{nC}_{12}\right)$ with varying concentrations of nC12 were studied. In addition to this, the mHEX algorithm was also validated with respect to ternary mixtures: three different compositions of methane $\left(\mathrm{nC}_{1}\right)$-butane $\left(\mathrm{nC}_{4}\right)$ $\mathrm{nC}_{12}$, and one composition of 1,2,3,4-tetrahydronaphthalene (THN)- $\mathrm{nC}_{12-}$ isobutylbenzene (IBB). For the binary mixtures studied here, our findings were in a good agreement with previous work in the literature, i.e., the components in the mixture show less tendency to segregate as the concentration of heavy component in the mixture increases. Additionally, in agreement with the literature, the heavier component separates to the cold side whereas the lighter component separate to the hot side. In ternary mixtures, the mHEX algorithm performs much better than regular heat exchange algorithm (HEX) in predicting the direction and magnitude of the thermodiffusive separation. Once again, the heaviest and the lightest components clearly separate to the cold and hot side, respectively. With respect to the ternary mixtures, the mHEX algorithm is about $17 \%$ more accurate in predicting the thermodiffusive separation than the regular HEX algorithm. It should be noted that all experimental data for comparison were obtained from microgravity environment. 


\subsection{Introduction}

A coupled mass and heat transport phenomenon caused by temperature difference at different locations in a homogeneous fluid mixture is called Soret effect/Thermodiffusion [89]. Thermodiffusion plays a significant role in various natural phenomena and numerous industrial applications including the oceanic thermohaline circulation [27], convection in stars [28], biomolecular binding [45, 56, 127], isotope separation in fluid mixtures [128, 129] and polymer characterization [14, 130]. Apart from these, thermodiffusion also plays an important role in the stratification of components in crude oil reservoirs [62, 131, 132].

Interest in thermodiffusion has spurred numerous experimental set-ups, as described in the review of Srinivasan and Saghir [60]. Apart from these, experimental investigations have also been made on reduced-gravity environment on board the international space station and free flying satellites $[131,133,134]$. This is because thermodiffusion is a very delicate phenomenon and small perturbations like free convection fields or undesired mechanical vibrations/disturbances in the experimental set-ups can easily eliminate this phenomenon [60].

Theoretical approaches to study thermodiffusion have led to numerous thermodynamics and physics based models as described in details by Srinivasan and Saghir [89]. However, these models often contradict each other and demonstrate a huge sensitivity towards the choice of equation of states (EOS) and thermodynamic properties [49]. Apart from these, thermodiffusion models have also been proposed using the principles of artificial neural networks [69, 108] and simple algebraic expressions [89]. While the neural network models are fairly accurate, they are unable to explain the physics behind the separation process. On the other hand, the algebraic expressions are empirical models that relay on the experimental data for formulation.

A major challenge in the above models is that they are unable to account for the complex inter-particle interactions that happen at the molecular level. This can be critical to the 
development of the understanding of the separation process in thermodiffusive flows. Molecular dynamics is a technique that addresses this requirement [78, 84, 124, 135$137]$.

Molecular dynamics techniques can be broadly classified into two major types, namely, equilibrium molecular dynamics (EMD) [111], and non-equilibrium molecular dynamics (NEMD) [112]. In EMD, the systems' dynamic properties are predicted via Green-Kubo relations in which the integral of the autocorrelation of flow quantities are related to the dynamic properties of the system that is devoid of any perturbed fields. In NEMD, the system's transport properties are calculated in the presence of external or agitating fields. Applying NEMD methods to study thermally activated fluids has resulted into three types of NEMD methods: synthetic non-equilibrium molecular dynamics (SNEMD) [70,116], reverse non-equilibrium molecular dynamics (RNEMD) [75, 117], and heat exchange algorithm (HEX) [73, 74].

The HEX algorithm is a very popular method that is often applied to study heat conduction in liquids because of the simplicity with which it can be implemented. More precisely, in this algorithm, the computational domain is divided further into subdomains, and periodically, a finite amount of kinetic energy is removed from one subdomain and added to the other. This is done by maintaining the centre of mass of the subdomains and employing velocity rescaling to adjust the non-translational kinetic energy. While the algorithm is quite popular, there are issues with its energy conservation that arise due to the leading-order truncation of $O\left(\Delta \mathrm{t}^{3}\right)$ of the coordinates in the Velocity Verlet integration scheme [34]. This leads to significant energy drift even in simulations for a few nanoseconds. Put differently, these errors restrict the simulation time scale to certain critical values beyond which the energy loses are too high to be neglected. We recently presented an updated algorithm (mHEX), that significantly subdues this drawback and enhances the computational speed [78]. The mHEX algorithm was validated with respect to the experimental data of several binary mixtures to prove its accuracy. 
In this work, the mHEX algorithm is applied to study the effect of compositional variation in two binary mixtures, namely, hexane $\left(\mathrm{nC}_{6}\right)$-decane $\left(\mathrm{nC}_{10}\right)$ and $\mathrm{nC}_{6}$-dodecane $\left(\mathrm{nC}_{12}\right)$. For each mixture, several compositions are studied to understand the effect on thermodiffusive separation. Additionally, thermodiffusion has also been studied in two ternary mixtures, namely, methane $\left(\mathrm{nC}_{1}\right)$-butane $\left(\mathrm{nC}_{4}\right)-\mathrm{nC}_{12}$, and 1,2,3,4tetrahydronaphthalene ( $\mathrm{THN}$ )- $\mathrm{nC}_{12}$-isobutylbenzene (IBB). While three compositions are considered for the former, one composition is considered for the latter. Comparisons have been made with experimental data as well as estimates from the HEX algorithm.

In the ensuing sections, molecular dynamics formulations are described (Sec. 3. 2), followed by computational cases in Section 3. 3. Section 3. 4 presents the analysis of the results and finally, pertinent conclusions are drawn in Section 3. 5.

\section{2. Thermodiffusion Using Molecular Dynamics}

\section{2.1. mHEX Algorithm}

To define a temperature gradient in the domain, heat flux is introduced via velocity rescaling of the particles at particular locations in the system. As mentioned previously in section 2.2, this will result in a linear temperature gradient that will in turn induce a concentration gradient in the domain. At the steady state, the thermodiffusion factor $\left(\alpha_{T}\right)$ is calculated as:

$\alpha_{T}=-\frac{T}{x_{1}\left(1-x_{1}\right)} \frac{\nabla x_{1}}{\nabla T}$

where $T$ is the mean temperature in the domain, $\nabla x$ is the spatial gradient of the mole fraction and $\nabla T$ is the spatial gradient of the temperature. 
As previously mentioned in section 2.3.2 and as in the HEX algorithm, in the mHEX algorithm, the two-step Velocity Verlet scheme is used for the time integration. A key aspect of this scheme is the velocity rescaling equation that is given by

$\bar{V}_{i}=(1-\zeta) V_{i}+\zeta V_{\Gamma_{k}}$

where $V_{i}$ and $\bar{V}_{i}$ are the velocity before and after rescaling, respectively, for the $i^{\text {th }}$ particle in the domain at a given time step. $V_{\Gamma_{k}}$ is the barycentric velocity of the particles in the region $\Gamma_{k}$. In mHEX algorithm, the rescaling factor, is based on the mixture constituents as:

$$
\zeta= \pm 0.004 \frac{T k_{b}}{\sqrt[n]{\left(\varepsilon_{i i} \varepsilon_{j j} \ldots \varepsilon_{n n}\right)}}\left[\frac{\sum_{k=1}^{n}\left(x_{k} M_{k}\right)}{\left(\sigma_{i i}+\sigma_{j j}+\ldots \sigma_{n n}\right)^{3}} \frac{n}{\rho N_{A}}\right]^{\frac{1}{3}}
$$

where $T$ is the temperature, $\rho$ is the density of the mixture, $N_{A}$ is the Avogadro number and $k_{B}$ is the Boltzmann constant. Also, for the $k^{\text {th }}$ component of the mixture, the mole fraction and the molecular weight are designated as $x_{k}$ and $M_{k}$, respectively. Finally, $\varepsilon_{i i}$ and $\sigma_{j j}$ are the depth of the potential well and the atomic diameter, respectively of the pure component species in the mixture.

A key highlight of the mHEX algorithm is that is calculated exactly once at the beginning of the algorithm. On the other hand, in the HEX algorithm, depends upon the particles in the individual zone and as a result is a $O\left(N_{p}\right)$ calculation is performed every time the velocity rescaling is applied, $N_{p}$ being the size of the system. Thus, the mHEX algorithm is computational much faster, yielding savings of about $8 \%$ on the CPU time.

\section{2.2. Computational Implementation \& Details}


As mentioned in sections 2.3.2 before, in implementing the Velocity-Verlet scheme, modelling the forces exerted on each particle due to its interaction with other particles in the system is the most critical and time-consuming part of a molecular dynamics simulation. Here, the interaction potential, was modelled via the summation of simple Lennard-Jones (LJ) pair potential with cut off ratio of $r_{c}=2.5 \sigma_{i j}$. This L-J potential as previously mentioned in section 2.3 .2 is given by:

$$
\phi\left(r_{i j}\right)=4 \varepsilon_{i j}\left[\left(\frac{\sigma_{i j}}{r_{i j}}\right)^{12}-\left(\frac{\sigma_{i j}}{r_{i j}}\right)^{6}\right]
$$

As mentioned in section 2.3.2 in the above equation, to obtain the potential parameters between dissimilar particles, the Lorentz-Berthelot mixing rules prescribed by Allen and Tildesley [122] have been applied:

$$
\begin{aligned}
& \sigma_{i j}=0.5\left(\sigma_{i i}+\sigma_{i j}\right) \\
& \varepsilon_{i j}=\sqrt{\varepsilon_{i i} \varepsilon_{j j}}
\end{aligned}
$$

where the LJ parameters of the identical particles are obtained from the literature and are reported in Table 3.1.

As previously explained in section 2.3.1 for the computational implementation of the Velocity-Verlet scheme to study thermodiffusion, a cubic volume was chosen for the computational domain that was assimilated using 32 slabs of identical thickness in the direction of the desired heat flux. The front view of the three-dimensional setup is shown in Figure 3.1. In this domain, the particles were randomly positioned and their velocities were characterized via the Maxwell-Boltzmann distribution function at $30 \%$ below the desired temperature. The system was let to equilibrate through velocity rescaling to the desired temperature. The equilibration took $2 \times 10^{5}$ time steps with a time step size of $\Delta t^{*}=0.0008$. Subsequently, the equilibrated state was used as the starting point of the thermodiffusion simulations wherein heat flux was introduced into the system and the 
simulations were carried out for an additional $2 \times 10^{6}$ time steps. A periodic boundary condition was used on all the walls of the simulation box, and the minimum image convention was used to reduce the wall impacts. The system's temperature was controlled using a Gaussian thermostat.

Table 3.1: Lennard-Jones potential parameters obtained from Ref. [123, 138].

\begin{tabular}{|c|c|c|}
\hline Material & $\varepsilon / \mathrm{k}_{\mathrm{b}}(\mathrm{K})$ & $\sigma(\mathrm{nm})$ \\
\hline $\mathrm{nC}_{5}$ & 346 & 0.545 \\
\hline $\mathrm{nC}_{4}$ & 343 & 0.510 \\
\hline $\mathrm{nC}_{6}$ & 393 & 0.595 \\
\hline $\mathrm{nC}_{10}$ & 471 & 0.680 \\
\hline $\mathrm{nC}_{12}$ & 550 & 0.710 \\
\hline $\mathrm{IBB}$ & 542 & 0.584 \\
\hline $\mathrm{THN}$ & 598 & 0.608 \\
\hline
\end{tabular}
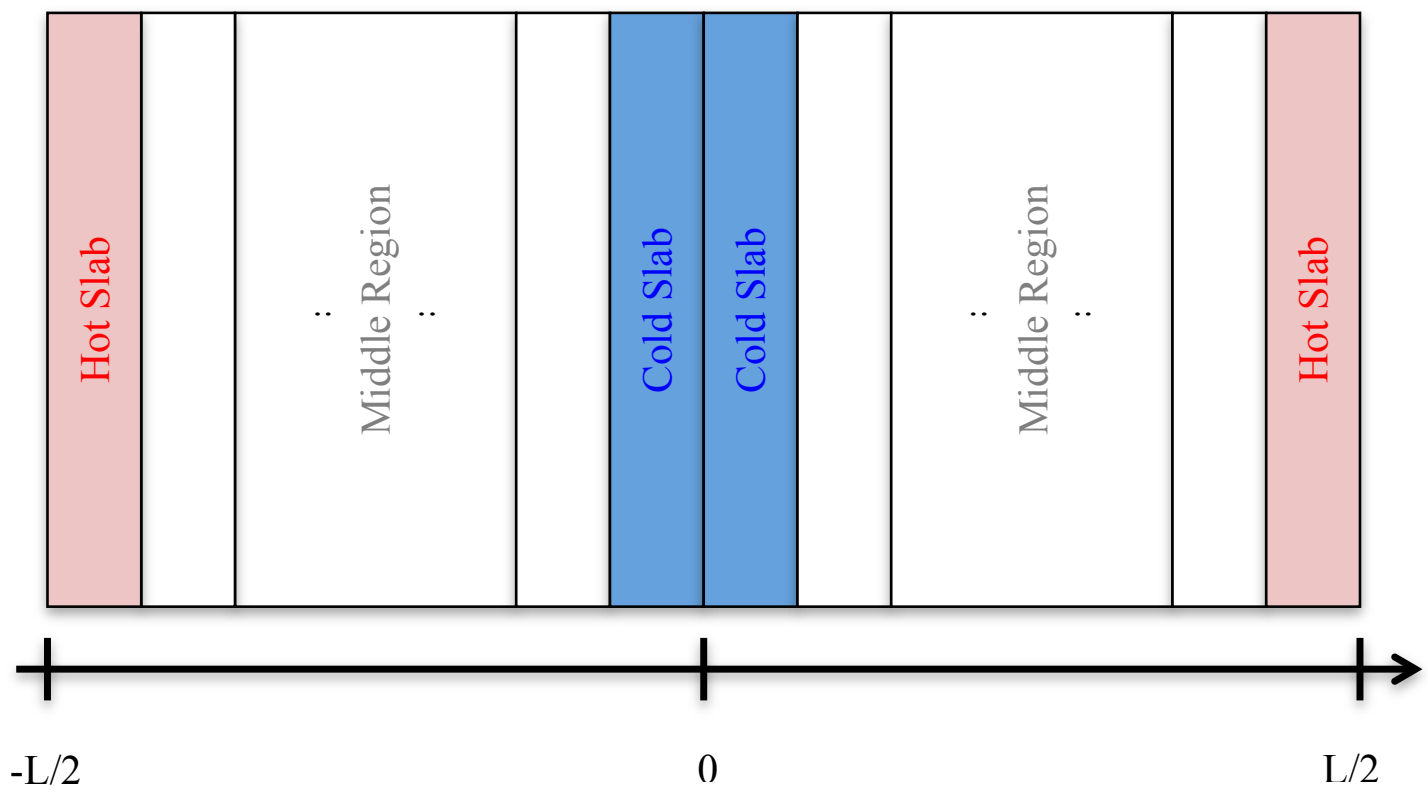

Fig. 3.1: Computational domain subdivided into slabs [139].

\subsection{Computational Cases}


Computational cases were designed for binary as well as ternary mixtures. Five different binary mixtures of $\mathrm{nC}_{6}-\mathrm{nC}_{12}$, with increasing composition of $\mathrm{nC}_{12}$ were considered. Similarly, five different mixtures of $\mathrm{nC}_{6}-\mathrm{nC}_{10}$ were considered with increasing mole fraction of $\mathrm{nC}_{10}$. These mixtures were at standard atmospheric pressure and at $\mathrm{T}=298 \mathrm{~K}$. The mixtures are summarized in Table 3.2.

Two different ternary mixtures were also studied, namely, $\mathrm{nC}_{1}-\mathrm{nC}_{4}-\mathrm{nC}_{12}$ and $\mathrm{nC}_{12}$-IBBTHN. For the former, three different compositions were considered (c.f. Table 3.2). The ternary mixtures were simulated at $35 \mathrm{MPa}$ and at $333 \mathrm{~K}$.

Each simulation case was repeated four times to minimize the statistical uncertainty due to the randomness involved in MD simulations. Throughout each simulation, the density of the mixture remained unchanged.

Table 3.2: Binary and ternary mixtures for which MD simulations were performed. All compositions are in mole fractions except for mixture \#14 for which the composition is in mass fraction [139].

\begin{tabular}{|c|c|c|}
\hline$\#$ & Mixture & Concentration \\
\hline 1 & $\mathrm{nC}_{6}-\mathrm{nC}_{12}$ & $\mathrm{nC}_{6}-0.9$ \\
\hline 2 & $\mathrm{nC}_{6}-\mathrm{nC}_{12}$ & $\mathrm{nC}_{6}-0.7$ \\
\hline 3 & $\mathrm{nC}_{6}-\mathrm{nC}_{12}$ & $\mathrm{nC}_{6}-0.5$ \\
\hline 4 & $\mathrm{nC}_{6}-\mathrm{nC}_{12}$ & $\mathrm{nC}_{6}-0.3$ \\
\hline 5 & $\mathrm{nC}_{6}-\mathrm{nC}_{12}$ & $\mathrm{nC}_{6}-0.1$ \\
\hline 6 & $\mathrm{nC}_{6}-\mathrm{nC}_{10}$ & $\mathrm{nC}_{6}-0.9$ \\
\hline 7 & $\mathrm{nC}_{6}-\mathrm{nC}_{10}$ & $\mathrm{nC}_{6}-0.7$ \\
\hline 8 & $\mathrm{nC}_{6}-\mathrm{nC}_{10}$ & $\mathrm{nC}_{6}-0.5$ \\
\hline 9 & $\mathrm{nC}_{6}-\mathrm{nC}_{10}$ & $\mathrm{nC}_{6}-0.3$ \\
\hline 10 & $\mathrm{nC}_{6}-\mathrm{nC}_{10}$ & $\mathrm{nC}_{6}-0.1$ \\
\hline 11 & $\mathrm{nC}_{1}-\mathrm{nC}_{4}-\mathrm{nC}_{12}$ & $\mathrm{nC}_{4}-0.1-\mathrm{nC}_{12}-0.7$ \\
\hline 12 & $\mathrm{nC}_{1}-\mathrm{nC}_{4}-\mathrm{nC}_{12}$ & $\mathrm{nC}_{4}-0.1-\mathrm{nC}_{12}-0.6$ \\
\hline 13 & $\mathrm{nC}_{1}-\mathrm{nC}_{4}-\mathrm{nC}_{12}$ & $\mathrm{nC}_{4}-0.1-\mathrm{nC}_{12}-0.4$ \\
\hline 14 & $\mathrm{nC}_{12}-\mathrm{IBB}_{-}-\mathrm{THN}_{1}$ & $\mathrm{IBB}^{-0.1,} \mathrm{THN}^{-0.8}$ \\
\hline
\end{tabular}




\subsection{Results \& Discussion}

In this section, we present the results from the simulation of the mixtures summarized in Table 3.2. The MD simulations of the binary mixtures listed in this table were done using the mHEX algorithm. The simulations for the ternary mixtures were done using the mHEX as well as the HEX algorithm. Additionally, for the ternary mixture, the results from the simulations were also compared with the experimental data.

\subsubsection{Binary Mixtures}

The thermodiffusion factors from the MD simulations of the two binary mixtures with different concentrations of $\mathrm{nC}_{6}$ are summarized in Table 3.3. The typical temperature profile inside the domain is shown in Figures 3.2a and $\mathrm{b}$ for mixtures \#1 and 6, respectively.
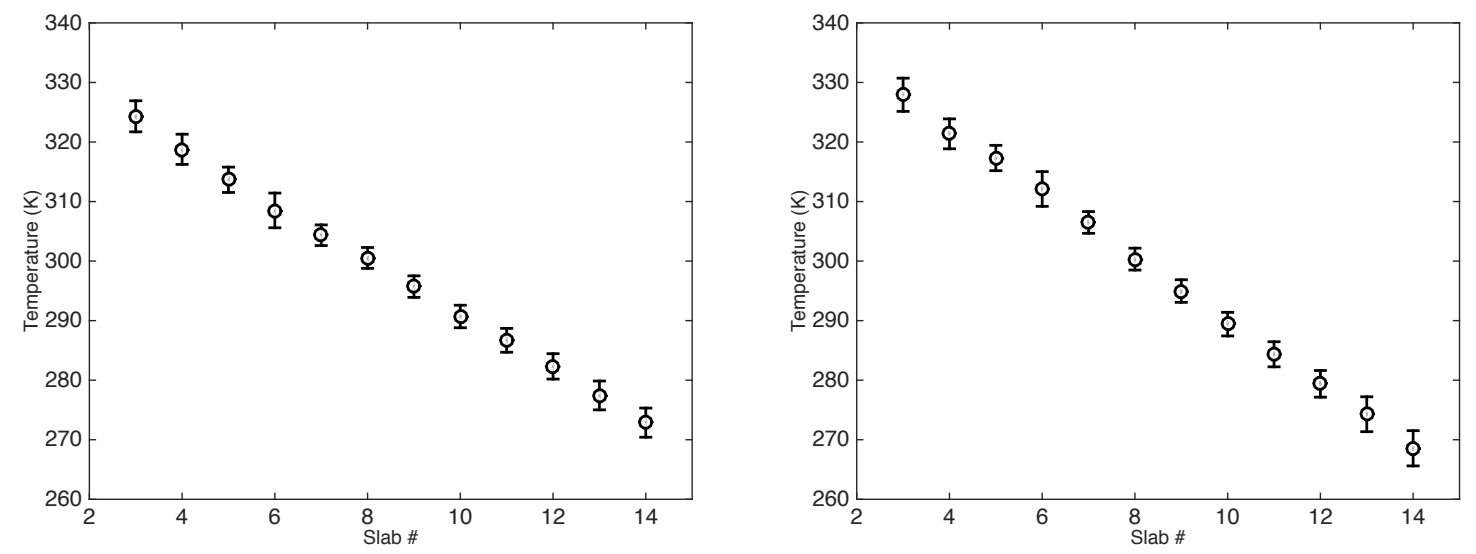

Fig. 3.2: (a, b) Typical temperature distribution in the domain in the $\mathrm{nC}_{6}-\mathrm{nC}_{10}$ and $\mathrm{nC}_{6}-\mathrm{nC}_{12}$ mixtures, respectively [139].

The distribution of $\mathrm{nC}_{6}$ in the domain for these two mixtures is shown in Figures 3.3a and b, respectively. As seen in these figures, with the establishment of temperature gradient, the lighter component in the mixture migrates towards the warmer zone. This will lead to the displacement of the heavier component in the respective mixture to the colder zones. Put differently, the thermodiffusive flows are such that the heavier component migrates 
to the cold side and the lighter component migrates to the hot side. This trend was observed for all the binary mixtures investigated in this study.

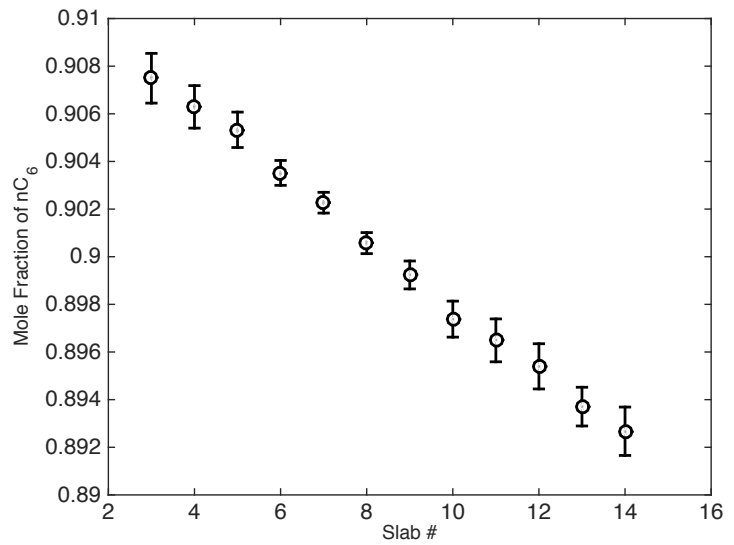

(a)

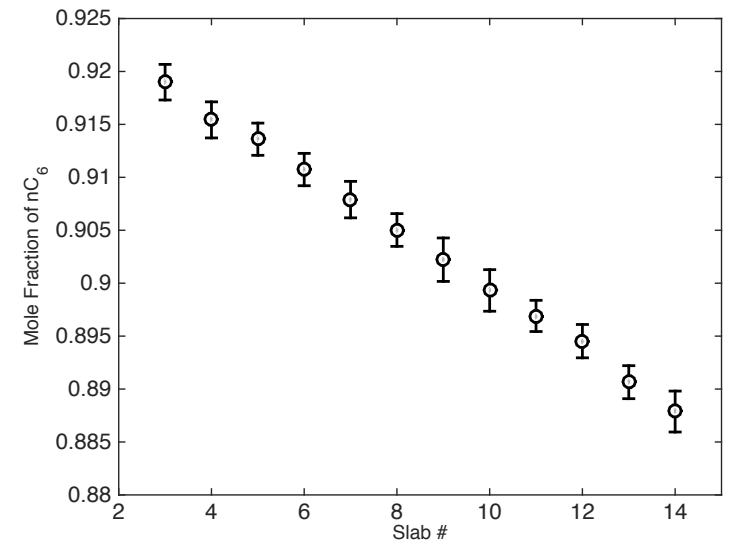

(b)

Fig. 3.3: (a, b) Distribution of $\mathrm{nC}_{6}$ in the domain in the $\mathrm{nC}_{6}-n \mathrm{C}_{10}$ and $\mathrm{nC}_{6}-\mathrm{nC}_{12}$ mixtures, respectively. In both mixtures, the mole fraction of $\mathrm{nC}_{6}=0.9[139]$.

As mentioned earlier, the strength of this separation is measured quantitatively using the thermodiffusion factor, $\alpha_{\mathrm{T}}$. The values of $\alpha_{\mathrm{T}}$ for these mixtures are plotted in Figure 3.4. As seen in this figure (c.f. Fig. 3.4), for both types of binary mixtures, $\alpha_{\mathrm{T}}$ decreases as the concentration of heavy component in the mixture increases. This decreasing trend indicates that the strength of thermodiffusive separation diminishes as the concentration of the heavier component in the mixture increases. This is expected and is due to the fact that the amount of energy required to displace and move the heavier component is higher and so as its concentration increases, the mobility of the components in the mixture progressively decrease.

Another observation that can be made from Figure 3.4 is that for any mole fraction, the thermodiffusive separation in the $\mathrm{nC}_{6}-\mathrm{nC}_{12}$ mixture is larger than in the $\mathrm{nC}_{6}-\mathrm{nC}_{10}$ mixture. In other words, thermodiffusion is stronger in mixture where there is a larger disparity between the two components in terms of the molecular weight. 
The trend lines have shown in Figure 3.4 present a linear relationship between the concentration of the heavier component in the mixture and the thermodiffusion factor. More precisely, the relationships for the two binary series studied in this work are:

$$
\begin{aligned}
& \alpha_{T_{n C_{12}}}=-0.720 x_{T_{n C_{12}}}+1.408 \\
& \alpha_{T_{n C_{10}}}=-0.425 x_{T_{n C_{10}}}+1.023
\end{aligned}
$$

From these relations if we extrapolate and find the values of $\alpha_{\mathrm{T}}$ at $x=0$ and $x=1$ and study the ratio, then we find that

$$
\frac{\left(\frac{\alpha_{T_{x=0}}}{\alpha_{T_{x=1}}}\right)_{n C_{12}}}{\left(\frac{\alpha_{T_{x=0}}}{\alpha_{T_{x=1}}}\right)_{n C_{10}}}=\frac{\left(\frac{M_{n C_{12}}}{M_{n C_{6}}}\right)}{\left(\frac{M_{n C_{10}}}{M_{n C_{6}}}\right)}
$$

This behaviour is consistent with the postulates of Galliero et al. [86].

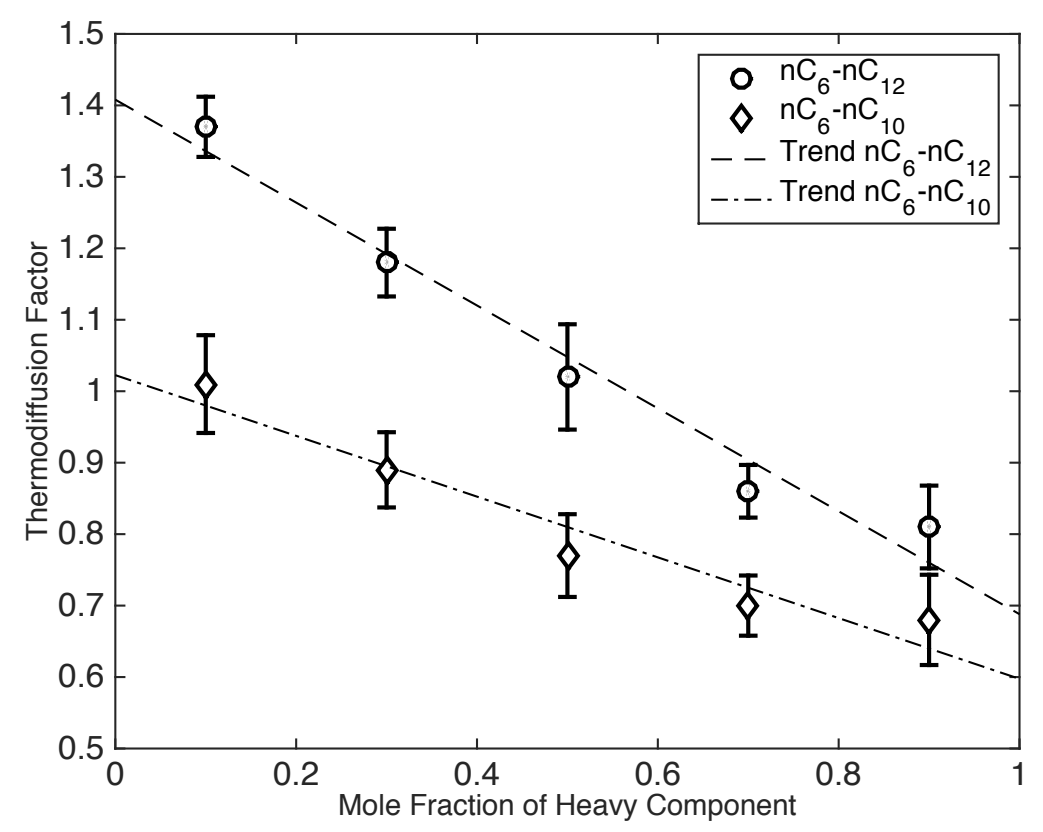

Fig. 3. 4: Thermodiffusion factors in binary mixtures [139]. 
Table 3.3: Thermodiffusion factor of the binary mixtures [139].

\begin{tabular}{|c|c|c|c|}
\hline$x_{n C_{6}}$ & $\alpha_{T}$ & $x_{n C_{6}}$ & $\alpha_{T}$ \\
\hline \multicolumn{2}{|c|}{$\mathrm{nC}_{6}-\mathrm{nC}_{12}$} & \multicolumn{2}{|c|}{$\mathrm{nC}_{6}-\mathrm{nC}_{10}$} \\
\hline 0.1 & $1.37 \pm 0.08$ & 0.1 & $1.01 \pm 0.13$ \\
\hline 0.3 & $1.18 \pm 0.09$ & 0.3 & $0.89 \pm 0.1$ \\
\hline 0.5 & $1.02 \pm 0.14$ & 0.5 & $0.77 \pm 0.11$ \\
\hline 0.7 & $0.86 \pm 0.07$ & 0.7 & $0.7 \pm 0.08$ \\
\hline 0.9 & $0.81 \pm 0.11$ & 0.9 & $0.68 \pm 0.12$ \\
\hline
\end{tabular}

${ }^{\mathrm{a}}$ All error bars related to repeatability errors for MD approaches.

\section{4.2. Ternary Mixtures}

The validated mHEX algorithm is applied to four ternary mixtures. Specifically, three compositions of $\mathrm{nC}_{1}-\mathrm{nC}_{4}-\mathrm{nC}_{12}$ at $\mathrm{T}=333 \mathrm{~K}$ and $\mathrm{P}=35 \mathrm{MPa}$ were studied. Apart from these, a ternary mixture of $\mathrm{nC}_{12}$-IBB-THN was also studied. The composition of the individual mixture is summarized in Table 3.2. The average temperature distribution inside the middle slabs for mixture \#11 is shown in Figure 3.5.

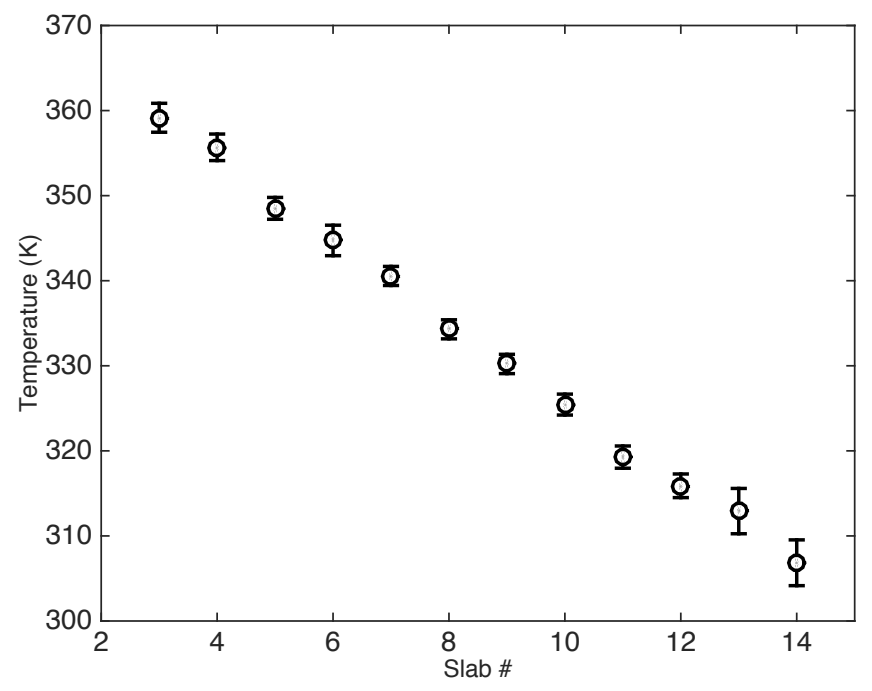

Fig. 3.5: Temperature distribution in the domain in mixture \#11 [139].

The concentration profiles of $\mathrm{nC}_{4}$ and $\mathrm{nC}_{12}$ can be observed in the Figures 3.6a and $\mathrm{b}$, respectively. As seen in these figures, $\mathrm{nC}_{12}$ moves towards the colder side whereas $\mathrm{nC}_{4}$ 
moves to the hot side. This trend is similar to the binary mixtures in which the heavier component moves to the cold side whereas the lighter component moves to the hot side.

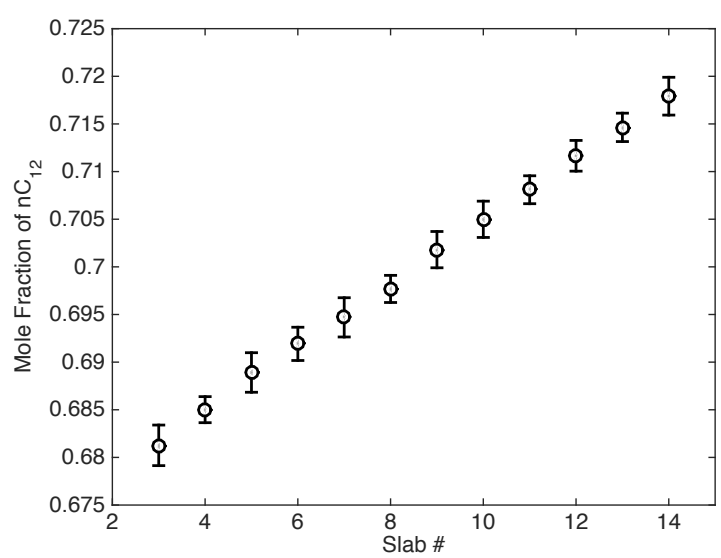

(a)

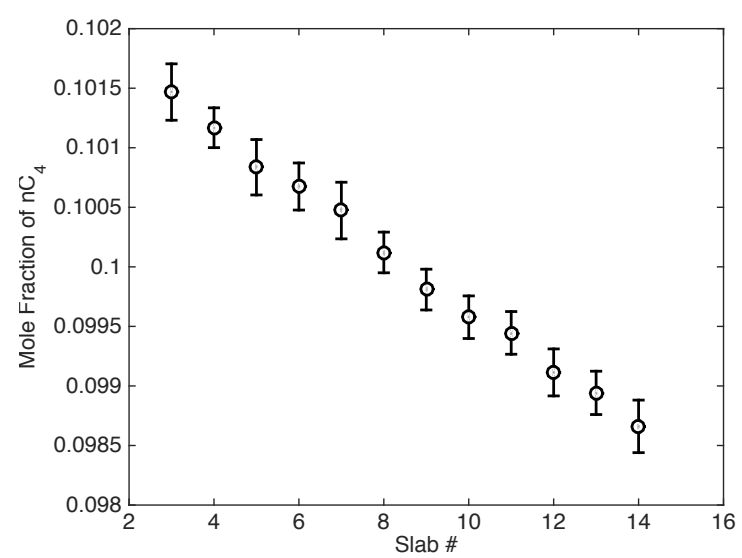

(b)

Fig. 3.6: (a, b) Distribution of $\mathrm{nC}_{12}$ and $\mathrm{nC}_{4}$, respectively, in the domain in mixture \#11[139].

The estimated value of thermodiffusion factor using the mHEX algorithm for each component in mixtures \#11-13 are shown in Figure 3.7. As seen in this figure, $\mathrm{nC}_{1}$ and $\mathrm{nC}_{12}$, the lightest and the heaviest components in the mixtures, respectively, have the largest magnitude of thermodiffusion factors. Put differently, the heaviest and the lightest components have a strong separation to the cold and hot side, respectively. $\mathrm{nC}_{4}$, which has an intermediate molecular weight, has a moderate value of $\alpha_{T}$. This is because, at the molecular level, in its interactions with the heavier component, i.e., $\mathrm{nC}_{12}$, it separates to the hot side. On the other hand, when it interacts with the lighter component, i.e., $\mathrm{nC}_{1}$, it separates towards the cold side. This is consistent with the observations made in the literature $[85,134]$.

The values of $\alpha_{T}$ for these three mixtures are summarized in Table 3.4. In this table, positive numbers indicate that the component migrates towards the cold side, whereas negative values indicate that the component moves towards the hot side. Comparing the values of the thermodiffusion factor with the experimental values reported by Srinivasan and Saghir [134], it is seen that the values predicted by the mHEX algorithm is in good agreement with the experimental data. On the other hand, the results from the predictions 
of the HEX algorithm are further away from the experimental data. This higher accuracy of the mHEX algorithm is consistent with the performance of this algorithm for several other the binary mixtures as reported by Mozaffari et al. [78]. This superior performance is attributed to the fact that the energy conservation is modelled more accurately in the mHEX algorithm than in the HEX algorithm, leading to a more accurate simulation of the inter-particle interactions.

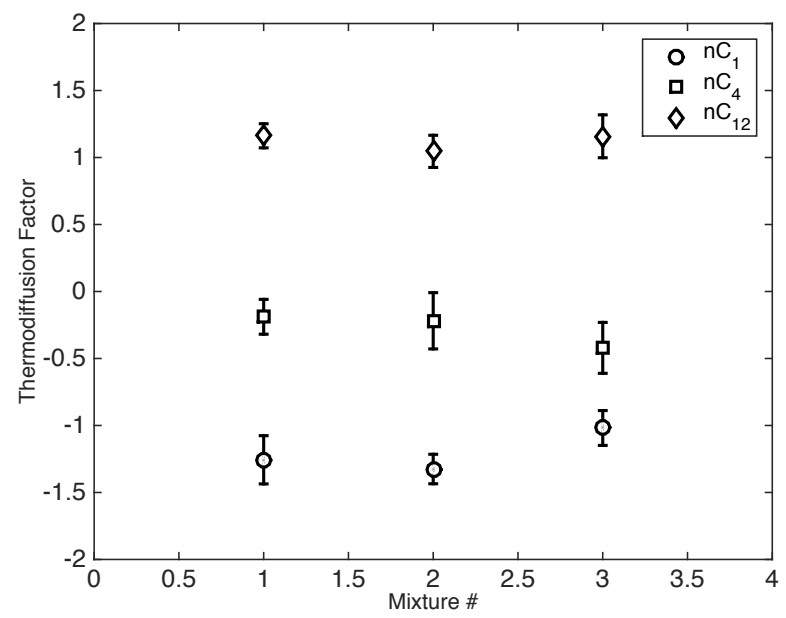

Fig. 3.7: Thermodiffusion factors in the ternary mixtures \#11-13 [139].

Table 3.4: Thermodiffusion factors in $\mathrm{nC1}$-nC4-nC12 mixtures. The experimental data is from Srinivasan and Saghir [134]. The HEX results are from the work of Galliero et al. [85]. The mHEX results are from Mozaffari et al. $[139]^{\mathrm{a}}$.

\begin{tabular}{|c|c|c|c|}
\hline Mixt. \# & mHEX & HEX & Expt. \\
\hline \multicolumn{3}{|c|}{$\mathrm{nC}_{12}$} \\
\hline 11 & $1.16 \pm 0.09$ & $1.05 \pm 0.03$ & 1.26 \\
\hline 12 & $1.05 \pm 0.12$ & $1.04 \pm 0.03$ & 1.2 \\
\hline 13 & $1.15 \pm 0.16$ & $1.17 \pm 0.04$ & 1.3 \\
\hline \multicolumn{3}{|c|}{$\mathrm{nC}_{1}$} \\
\hline 11 & $-1.26 \pm 0.18$ & $-0.88 \pm 0.03$ & -1.53 \\
\hline 12 & $-1.32 \pm 0.11$ & $-0.94 \pm 0.03$ & -1.55 \\
\hline 13 & $-1.02 \pm 0.13$ & $-0.87 \pm 0.04$ & -1.2 \\
\hline
\end{tabular}

\footnotetext{
${ }^{\mathrm{a}}$ The error bars in MD techniques are due to repeatability. The experimental method was conducted in microgravity environment only once.
} 
To validate the superior performance of the mHEX algorithm, another ternary mixture of $\mathrm{nC}_{12}$-IBB-THN at atmospheric temperature and pressure has also been considered. The composition of this mixture is summarized in Table 3.2. The typical profile of the temperature distribution is shown in Figure 3.8.

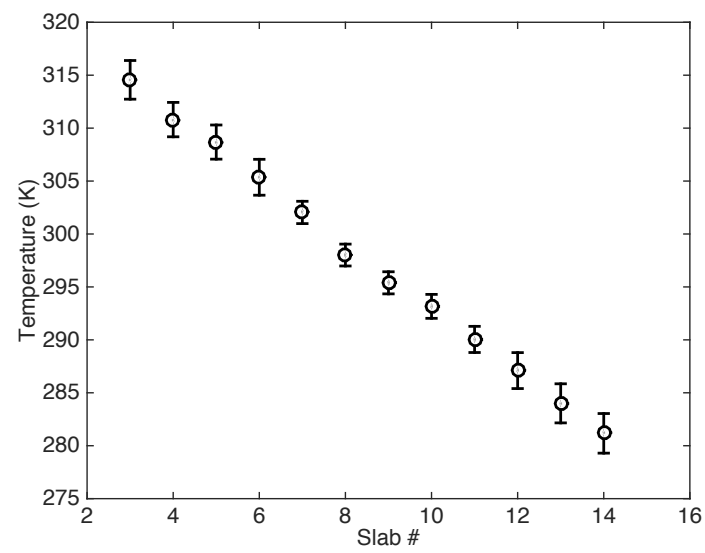

Fig. 3. 8: Temperature distribution in the domain in mixture \#14 [139].

Also, the concentration distribution of IBB and THN is shown in Figures 3.9a-b, respectively. As seen in these figures, as the thermal gradient is established in the region, IBB tends to migrate towards the hot side whereas THN migrates towards the cold side.

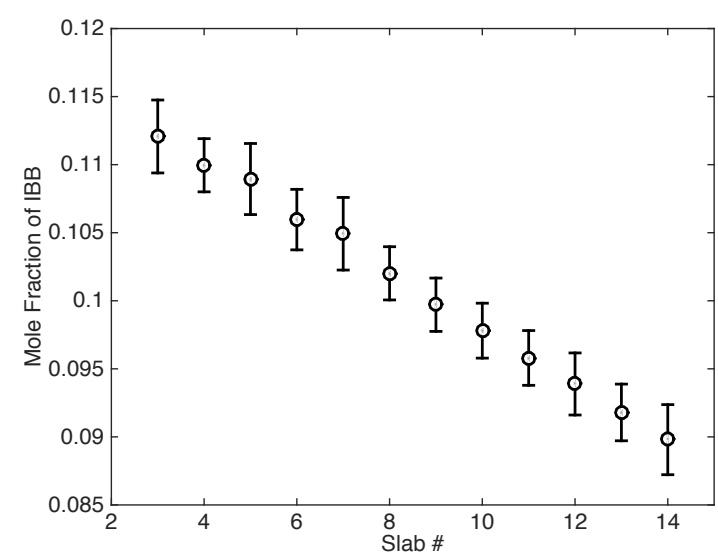

(a)

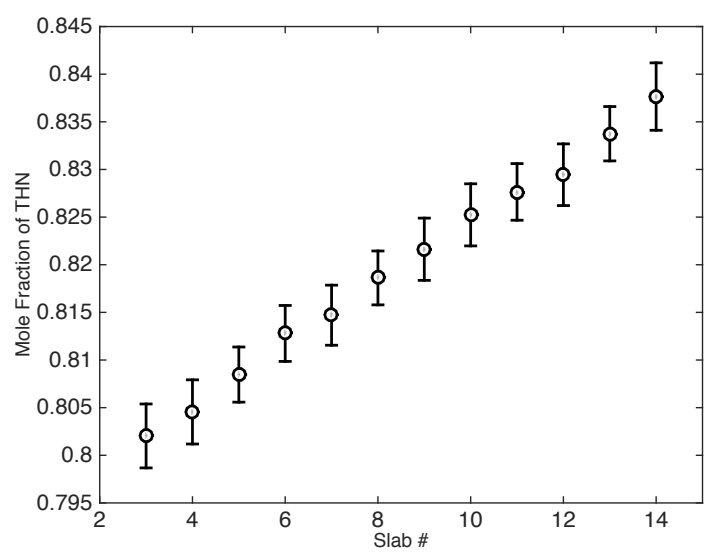

(b)

Fig. 3. 9: (a, b) Distribution of IBB and THN, respectively, in the domain in mixture \#14 [139]. 
The results from the mHEX as well as HEX algorithms are summarized in Table 3.5 along with the experimental data from Ahadi and Saghir [131] As seen in this table, both algorithms are able to predict the direction of separation, in agreement with the experimental data. However, the strength of the separation, indicated by the magnitude of these values is more accurate in the mHEX algorithm than in the HEX algorithm. More precisely, regular HEX algorithm predictions of Soret coefficients are quite erroneous with relative errors of about $39 \%, 44 \%$ and $30 \%$ for $\mathrm{THN}, \mathrm{IBB}$ and $\mathrm{nC}_{12}$, respectively. On the other hand, with mHEX algorithm these relative errors are approximately $14 \%$, $15 \%$ and $19 \%$ for $\mathrm{THN}, \mathrm{IBB}$ and $\mathrm{nC}_{12}$, respectively. Once again, these results establish that the modified algorithm is suitable to study thermodiffusion in ternary mixtures as well.

Table 3.5: Soret Coefficients $(1 / \mathrm{K}) \times 10^{4}$ in $\mathrm{nC}_{12}$-IBB-THN mixture. The experimental data is from Ahadi and Saghir [132]. The HEX and mHEX are from Mozaffari et al. [139] ${ }^{\mathrm{a}}$.

\begin{tabular}{|c|c|c|c|}
\hline Comp. & Expt. & mHEX & HEX \\
\hline IBB & $-8.15 \pm 1.2$ & $-6.93 \pm 0.8$ & $-4.57 \pm 0.09$ \\
\hline $\mathrm{THN}$ & $13.69 \pm 0.09$ & $11.71 \pm 1.1$ & $8.27 \pm 0.09$ \\
\hline $\mathrm{nC}_{12}$ & $-5.66 \pm 0.6$ & $-4.61 \pm 1.3$ & $-3.99 \pm 0.09$ \\
\hline
\end{tabular}

${ }^{\mathrm{a}}$ The error bars in MD techniques are due to repeatability while for experimental method represents the repeatability as well as of the instruments errors.

\section{5. Summary \& Conclusions}

In this study, a recently proposed mHEX algorithm that has been validated with respect to binary hydrocarbon mixtures has been used to study thermodiffusion in two different types of binary mixtures, namely, $\mathrm{nC}_{6}-\mathrm{nC}_{10}$ and $\mathrm{nC}_{6}-\mathrm{nC}_{12}$, with varying compositions of the constituents. Additionally, we also evaluate this algorithm with respect to the regular $\mathrm{HEX}$ algorithm by applying it to two ternary mixtures, namely, $\mathrm{nC}_{1}-\mathrm{nC}_{4}-\mathrm{nC}_{12}$ and $\mathrm{nC}_{12-}$ IBB-THN. Further, for the former mixture, three different compositions at $\mathrm{T}=333 \mathrm{~K}$ and $\mathrm{P}=35 \mathrm{MPa}$ were considered. The second mixture was at atmospheric temperature and pressure. 
From the results, the following observations and conclusions were made:

1. Binary mixtures: In general, the heavier component separates to the cold side whereas the lighter component separates to the hot side. The strength of separation of separation, indicated by the magnitude of the thermodiffusion factor, becomes weaker as the concentration of the heavier component in the mixture increases.

2. Ternary mixtures: In the normal ternary mixtures of $n C 1-n C 4-n C 12$, the heaviest component accumulated in the cold zone, whereas the other two components moved to hot zone. Similarly, in the ternary mixture of nC12-IBB-THN, THN migrated towards cold region, whereas IBB and $\mathrm{nC} 12$ gathered in hot regions. This separation trend is consistent with the experimental observations.

3. mHEX algorithm: The new mHEX improved the prediction of thermodiffusion factors over its regular counterpart algorithm for all mixtures. The associated relative errors of mHEX algorithm with respect to experimental data obtained from microgravity environment for the studied ternary mixtures were about $17 \%$ more accurate than the estimates of thermodiffusion factor from the HEX algorithm. 


\section{CHAPTER 4- A MODIFIED HEAT EXCHANGE ALGORITHM TO STUDY THERMO-SOLUTAL DIFFUSION IN LIQUID MIXTURES}

This chapter is based on a submitted paper to the Journal of Thermal Science

Mozaffari, S. H., Srinivasan, S. \& Saghir, M. Z.,

A modified heat exchange algorithm to study thermo-solutal diffusion in liquid mixtures, Submitted to Int. Therm. Sci.

\subsection{Summary}

A modified heat exchange algorithm is proposed to perform N-body molecular dynamics investigations. 42 different case studies involving binary mixtures have been conducted in which the algorithm has been applied to study coupled heat and mass transport using the principles of molecular dynamics. Comparisons have been made with experimental data as well as the molecular dynamics approach using the traditional heat exchange algorithm. It has been shown that the modified algorithm has significantly better energy conservation properties, is more accurate, and is about $9 \%$ more computationally efficient than the traditional heat exchange algorithm.

\subsection{Introduction}

The problem of understanding the effect of non-uniform thermal field on the separation processes in a fluid mixture is an important scientific computation that is relevant to many industrial and natural processes. Some of the industrial applications where this computation is relevant include isotope separation [140], trapping of DNA [141], thermal field flow fractionation devices for characterizing polymers and colloidal systems [142], fluid transport in outer space [143], biomolecular binding curves [144] and freeze drying of food [145]. It is also relevant in natural processes such as salinity of ocean [146], solar ponds [147] and crude oil stratification in underground oil reservoirs [148-150]. This has resulted in scientific investigations involving thermal gradient related transport in gases [6,7], electrolytes [9], alcohols [10, 11], ferrofluids [18, 19], polymers [13, 15], proteins [25] as well as latex particles [23]. 
Researchers in theoretical fields have tried to comprehend this coupled mass and heat transport phenomenon thoroughly via thermodynamic principles [12, 47, 151-155]. However, the suggested theoretical models often contradict each other and their preciseness is limited to the proper choice of equation of state (EOS) [61]. On the hand, unwanted mechanical vibrations as well as inevitable gravitational fields can lead to erroneous experimental results on the ground conditions $[63,106]$. Consequently, interest in conducting the thermodiffusion experiment in micro-gravity environment has been increased in recent years [60, 131]. Similarly, different computational approaches including neural networks, finite volume and molecular dynamics (MD) have been applied to investigate the thermo-solutal diffusion [78, 80, 118, 156, 157]. In neural network methods, lack of enough experimental data to train the system for being able to predict its behaviour at new thermodynamic state is problematic. On the other hand, macro level models like control volume finite element deal with continuum problems and cannot be used directly to consider the behaviour of the system molecular scale. As a result, MD technique is the most popular technique to study thermodiffusion via consideration of complex inter-particle interaction at the molecular level.

The MD approaches can be classified into two major types: equilibrium molecular dynamics (EMD) [111], and non-equilibrium molecular dynamics (NEMD) methods [112]. EMD techniques predict the systems dynamic properties via Green-Kubo or Einstein formula wherein the integral of the autocorrelation of flow quantities are related to the dynamic properties of the system without perturbed fields. On the other hand, using NEMD methods, transport properties of the system can be estimated in the presence of agitating or external fields. NEMD methods used to study fluids involving heat conduction in literature can be broadly divided into three major groups including synthetic non-equilibrium molecular dynamics (SNEMD) [70], re-verse non-equilibrium molecular dynamics (RNEMD) [75], and heat exchange algorithm (HEX) [73]. These methods vary in their approach to generate heat fluxes.

In SNEMD methods, phenomenological coefficients are measured through linear response of the system to intentional deviations from equilibrium conditions in phase 
space induced by specific time-varying external force. In RNEMD, proposed by MüllerPlathe [75], after identifying hot and cold particles in the system, their momentums are simply swapped. Kuang and Gezelter [158] proposed a variation of this RNEMD approach by employing velocity rescaling instead of momentum swaps.

HEX algorithm, which is the focus of this study, was proposed by Ikeshoji and Hafskjold [73], and is a popular algorithm to investigate studies involving heat conduction. In this algorithm, the computational domain is subdivided into sub-domains and periodically; a certain amount of kinetic energy is removed from one sub-domain (source) and added to another subdomain (sink). In doing so, the individual subdomains centre of mass velocities are preserved and velocity rescaling is used to adjust the non-translational kinetic energy.

While this classical algorithm has been used widely since its introduction, studies have reported an issue with the energy conservation of this algorithm [74, 87]. This is due to the leading-order truncation errors of $O\left(\Delta t^{3}\right)$ of the coordinates in the Velocity Verlet integration scheme [88]. Specifically, significant amount of energy drift has been observed when the simulations are made for a time scale of few nanoseconds. This severely restricts the simulation time scales to critical values beyond which the energy losses are deemed unacceptable. While this could be solved using small time steps (e.g. $\left.\mathrm{O}\left(10^{-17} \mathrm{~s}\right)\right)$, the computational time can significantly increase. Another option could be to use an additional thermostat. However, this could impact the temperature profile that one would like to study [88].

In this work, we present a modified form of the HEX algorithm that subdues the energy drift leading to higher accuracy. Additionally, the modification results in higher computationally efficiency that can play a significant role in time saving of simulation of industrial multi-scale thermodiffusion models like crude oil stratification in an entire oil reservoir. The modified algorithm has been applied to study binary mixtures under the influence of imposed heat ux. More precisely, the effect of a thermal gradient on the separation of constituents in six different binary mixtures has been studied using the 
modified HEX (mHEX) algorithm. Additionally, comparisons have been made between the mHEX and the HEX algorithm with respect to each other as well as experimental data to evaluate the performance of the mHEX algorithm.

\subsection{Modified HEX (mHEX) Algorithm}

As mentioned previously in sections $2.3 .1 \& 3.2 .1$, the time integration scheme of the mHEX algorithm is the same as the HEX algorithm, namely, the two-step Velocity Verlet scheme. The key modification is in the velocity rescaling equation, the rescaling factor (६) in particular:

$\bar{V}_{i}=(1-\zeta) V_{i}+\zeta V_{\Gamma_{k}}$

where $V_{i}$ and $\bar{V}_{i}$ are the velocity before and after rescaling, respectively, for the $\mathrm{i}^{\text {th }}$ particle in the domain at a given time step. $V_{\Gamma_{k}}$ is the barycentric velocity of the particles in the region $\Gamma_{k}$. In mHEX algorithm, the rescaling factor, is based on the mixture constituents as:

$\zeta= \pm 0.008 \frac{T k_{b}}{\sqrt{\varepsilon_{i i} \varepsilon_{i j}}}\left[\frac{\sum_{k}\left(x_{k} M_{k}\right)}{\left(\sigma_{i i}+\sigma_{i j}\right)^{3}} \frac{1}{\rho N_{A}}\right]^{\frac{1}{3}}$

In above equation $T$ is the temperature, $\rho$ is the density of the mixture, $k_{B}$ is the Boltzmann constant, $N_{A}$ is the Avogadro number. $x_{k}$ and $M_{k}$ are mole fraction and the molecular weight, respectively, of the $k^{\text {th }}$ component of the mixture. $\sigma_{i i}$ and $\varepsilon_{i i}$ are the atomic diameter and depth of the potential well, respectively of the pure component species in the mixture. This is different from the original HEX algorithm in which the scaling factor depends upon the particles in the individual zone and as a result is a $O\left(N_{p}\right)$ calculation is performed every time the velocity rescaling is applied, $N_{p}$ being the size of the system. 
Thus, for the $i^{\text {th }}$ particle in the system, the steps of the Velocity Verlet implementation for mHEX algorithm are

$$
\begin{aligned}
& V_{i}^{n+\frac{1}{2}}=\bar{V}_{i}^{n}+\frac{f_{i}^{n}}{2 m_{i}} \Delta t \\
& r_{i}^{n+1}=r_{i}^{n}+\frac{1}{2} V_{i}^{n+\frac{1}{2}} \Delta t \\
& f_{i}^{n+1}=-\nabla_{r_{i}} \phi\left(r^{n+1}\right) \\
& V_{i}^{n+1}=V_{i}^{n+\frac{1}{2}}+\frac{f_{i}^{n+1}}{2 m_{i}} \Delta t \\
& \bar{V}_{i}^{n+1}=(1-\zeta) V_{i}^{n+1}+\zeta V_{\Gamma_{k}}^{n}
\end{aligned}
$$

In the last equation, $\zeta$ is a fixed value that is calculated using Eqn. (4.2) at the beginning of the simulation, outside the time loop of algorithm. As previously mentioned in sections 1.3.1, 2.3.2 and 3.2.2 the interaction potential function, $\phi$ is modelled using the simple Lennard-Jones (LJ) potential with a cut off ratio of $r_{c}=2.5 \sigma_{i j}$. This LJ potential is given by

$$
\phi\left(r_{i j}\right)=4 \varepsilon_{i j}\left[\left(\frac{\sigma_{i j}}{r_{i j}}\right)^{12}-\left(\frac{\sigma_{i j}}{r_{i j}}\right)^{6}\right]
$$

where, $\phi, \varepsilon_{i j}, \sigma_{i j}$ and $r_{i j}$ are pair potential $(\mathrm{J})$, well-depth potential (J), atomic diameter (m), and distance between particles (m). subscripts $i$ and $j$ denote dissimilar particles.

Furthermore, to obtain the potential parameter for dissimilar particles, the following Lorentz-Berthelot mixing rules have been applied [122]: 
$\sigma_{i j}=0.5\left(\sigma_{i i}+\sigma_{j j}\right)$

$\varepsilon_{i j}=\sqrt{\varepsilon_{i i} \varepsilon_{j j}}$

Where the LJ parameters of identical particles are obtained from the literature for different binary mixtures (c.f. Table 4.1).

Table 4.1: Lennard-Jones potential parameters. These parameters were obtained from NIST Thermophysical Properties of Hydrocarbon Mixtures Database [123].

\begin{tabular}{|c|c|c|c|c|c|c|}
\hline Material & $\mathbf{A r}$ & $\mathbf{K r}$ & $\mathbf{n C}_{\mathbf{5}}$ & $\mathbf{n C}_{\mathbf{6}}$ & $\mathbf{n C}_{\mathbf{1 0}}$ & $\mathbf{n C}_{\mathbf{1 2}}$ \\
\hline$\varepsilon / \mathrm{k}_{\mathrm{b}}(\mathrm{K})$ & 119.8 & 167 & 346 & 393 & 471 & 550 \\
\hline$\sigma(\mathrm{nm})$ & 0.341 & 0.363 & 0.545 & 0.595 & 0.680 & 0.710 \\
\hline
\end{tabular}

\subsection{Computational Setup and Equilibration}

As mentioned in the introduction section, the mHEX algorithm has been evaluated by studying the separation of constituents in the presence of a non-uniform thermal field in six different binary mixtures. For this, the computational domain is a cubic volume that was assimilated using 32 slabs of identical thickness in the direction of the desired heat flux. The front view of the three-dimensional setup is shown in Figure 4.1 (Previously explained in sections 3.2.2). As shown in the figure, the two end layers are the hot zones, whereas the two central layers are the cold zones. 


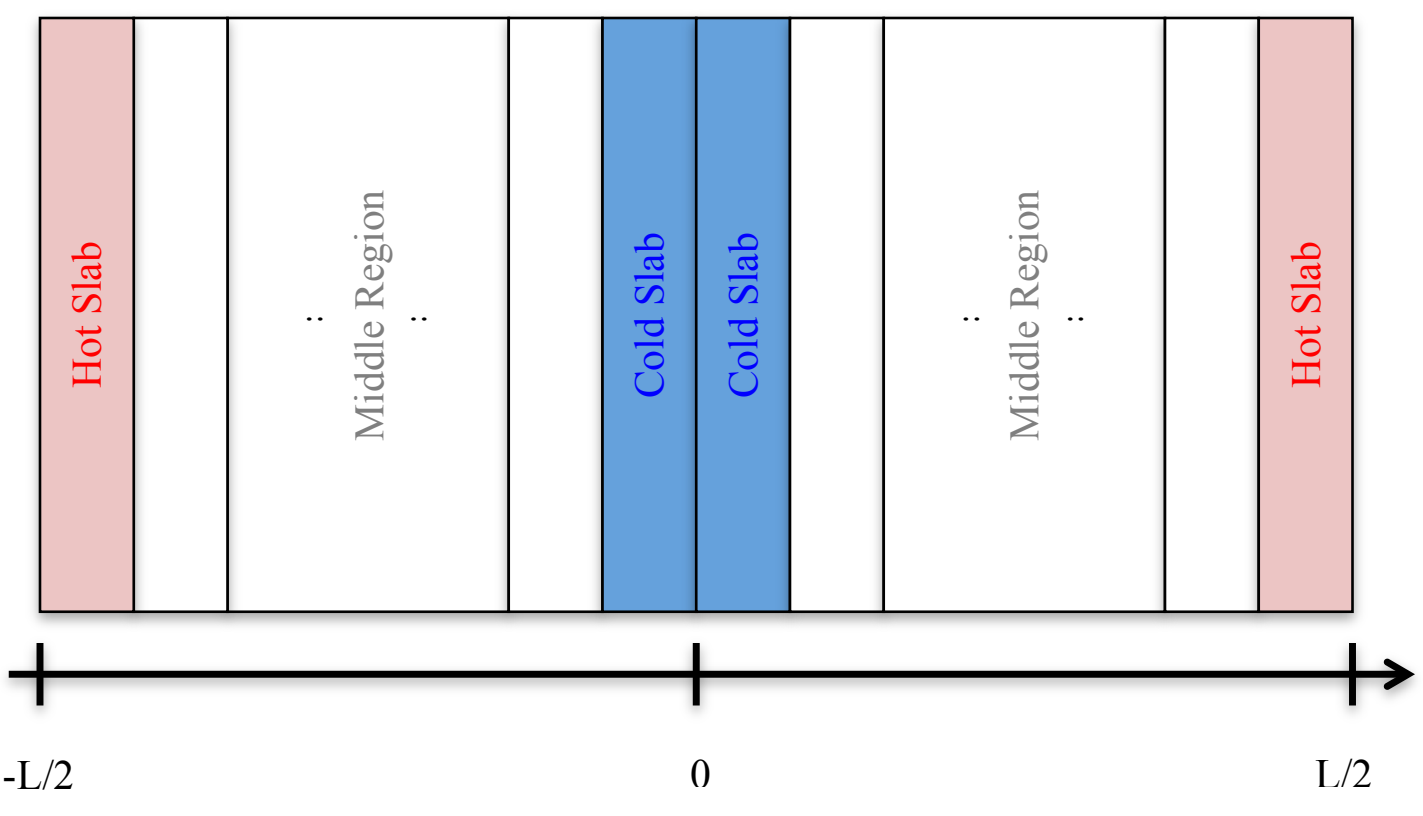

Fig. 4.1: Computational domain subdivided into slabs.

In all MD simulations, the particles were initially distributed with random coordinates. The velocities of the particles were characterized via the Maxwell-Boltzmann distribution function at $30 \%$ below the desired temperature. By intentionally setting the system temperature away from the desired temperature, we can increase the rate of equilibration. The system was let to equilibrate through velocity rescaling to the desired temperature. The equilibration took $2 \times 10^{5}$ time steps with a time-step size of $\mathrm{t}=0.002$ for the $\mathrm{Ar}-\mathrm{Kr}$ system and $t=0.0008$ for the hydrocarbon mixtures. The equilibrated state was used as the starting point of the thermodiffusion simulations wherein heat flux was introduced into the system and the simulations were carried out for an additional $1 \times 10^{6}$ time steps. A periodic boundary condition was used on all the walls of the simulation box, and the minimum image convention was used to reduce the wall impacts. The system's temperature was controlled using a Gaussian thermostat during the first 100,000 iterations.

Computational cases were considered to evaluate the following: (i) the accuracy of the modified algorithm, (ii) the computational speed with respect to the HEX algorithm and (iii) the effect of increasing the size of the system. To study these, the $42 \mathrm{MD}$ simulations that were conducted are summarized in Appendix B. Each case listed in this table was 
simulated four times, and the average of the results are presented in this work. The thermodynamic conditions of these mixtures are summarized in Appendix C.

\subsection{Simulation Results and Discussions}

The first step is the validation of the proposed algorithm and its comparison with the HEX algorithm. For this, the algorithm has been applied to study thermal-gradient induced separation in binary liquid mixtures. Specifically, in response to a temperature gradient along a domain, the constituents of a mixture separate to hot/cold zones, creating a concentration gradient. As previously mentioned in sections 2.2 and 3.2.1 the strength of this separation is characterized by the thermodiffusion factor $\left(\alpha_{T}\right)$ that is calculated as:

$\alpha_{T}=-\frac{T}{x_{1}\left(1-x_{1}\right)}\left(\frac{\nabla x_{1}}{\nabla T}\right)$

The estimate of $\alpha_{T}$ using the mHEX and HEX algorithm for the six mixtures are summarized in Table 4.2 alongside the experimental data from the literature. As seen in this table (Table 4.2), the modified algorithm proposed in this work fares superior to the traditional HEX algorithm. The only aberration seems to be the last mixture where the modified algorithm under performs compared to the HEX algorithm. However, the estimate with the error bar is still close to the experimental data.

The improved accuracy of algorithm can be attributed to the fact that scaling factor, $\zeta$, in mHEX is uniform throughout the simulation. On the other hand, in HEX algorithm, the fluctuations in $\zeta$ through the iterations are tremendous. Due to this, with HEX algorithm, the system experiences much stronger arbitrary disturbances when velocity rescaling is applied, introducing errors in the separation process in the domain. On the other hand, with the mHEX algorithm, due to the uniform value of $\zeta$, this issue is greatly subdued, resulting in more accurate calculations. The fluctuation of $\zeta$ in the Ar- $\mathrm{Kr}$ mixture is shown in Figure 4. 2, and is similar for the other mixtures. 


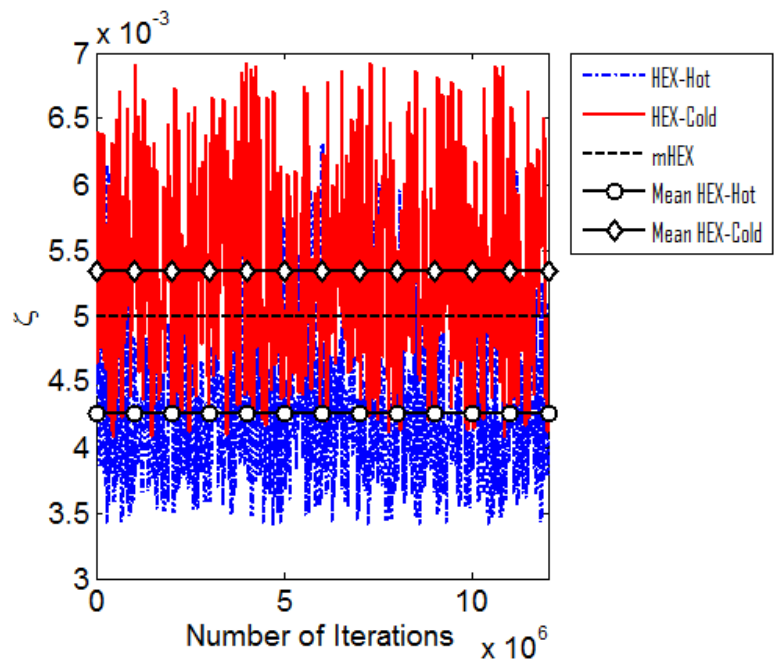

Fig. 4.2: Value of $\zeta$ in Ar-Kr mixture using the mHEX and HEX algorithms.

Another important feature of the mHEX algorithm is the improvement in its energy conservation property. In Figure 4. 3 we illustrate this with the energy loss in the $\mathrm{nC}_{5^{-}}$ $\mathrm{nC}_{10}$ mixture as a function of time. As seen in this figure (Figure 4.3), the loss is significantly lower in with the mHEX algorithm almost stabilizing at about half way through the simulation. On the other hand, the energy loss is continuous through the simulation with the HEX algorithm. The trend is similar in the other mixtures. In fact, the total energy loss at the end of the simulation for three mixtures is summarized in Figure 4. 4. As seen in this figure, irrespective of the size of the system, i.e., the number of particles (molecules) in the domain, the mHEX algorithm has much better energy conservation than the HEX algorithm. Further, in both algorithms, the energy conservation improves as the size of the system increases, approaching a plateau. Collectively, these results are evidence of the accuracy of the modified algorithm. For instance in small systems with only 400 particles, mHEX algorithm subdued energy loss by $17 \%, 21 \%$ and $23 \%$ for non-equimolar mixtures of $\mathrm{nC}_{6}-\mathrm{nC}_{10}, \mathrm{nC}_{6}-\mathrm{nC}_{12}$ and equimolar mixture of $\mathrm{nC}_{5}-\mathrm{nC}_{10}$, respectively with respect to $\mathrm{HEX}$ algorithm. While for the largest system with 3200 particles mHEX algorithm reduced the energy loss approximately by $50 \%$ for all mixtures. 
Table 4.2: Thermodiffusion factor estimated using the mHEX and HEX algorithm on a system of 1000 particles. The value in parentheses indicates the deviation from the experimental/ benchmark data in the reported reference.

\begin{tabular}{|c|c|c|c|}
\hline \multicolumn{3}{|c|}{$\alpha_{T}$} \\
\hline Mixture & $\mathrm{mHEX}^{\mathrm{a}}$ & HEX $^{\mathrm{a}}$ & Litrature $^{\mathrm{b}}$ \\
\hline $\mathrm{Ar}-\mathrm{Kr}$ & $1.91 \pm 0.13$ & $2.02 \pm 0.12$ & $1.85 \pm 0.11$ \\
& $(3.24 \%)$ & $(9.19 \%)$ & MD $^{[78]}$ \\
\hline $\mathrm{nC}_{5}-\mathrm{nC}_{10}\left(\mathrm{X}_{\mathrm{nC}_{5}}=0.2\right)$ & $0.98 \pm 0.17$ & $0.88 \pm 0.12$ & $1.14 \pm 0.27$ \\
& $(14.04 \%)$ & $(22.81 \%)$ & Expt. $^{[126]}$ \\
\hline $\mathrm{nC}_{5}-\mathrm{nC}_{10}\left(\mathrm{X}_{\mathrm{nC}_{5}}=0.5\right)$ & $0.95 \pm 0.12$ & $0.92 \pm 0.15$ & $0.98 \pm 0.23$ \\
& $(3.16 \%)$ & $(6.12 \%)$ & Expt. $^{[126]}$ \\
\hline $\mathrm{nC}_{5}-\mathrm{nC}_{10}\left(\mathrm{X}_{\mathrm{nC}_{5}}=0.8\right)$ & $1.18 \pm 0.25$ & $1.21 \pm 0.12$ & $1.06 \pm 0.25$ \\
& $(10.17 \%)$ & $(14.15 \%)$ & Expt. $^{[126]}$ \\
\hline $\mathrm{nC}_{6}-\mathrm{nC}_{10}$ & $0.83 \pm 0.17$ & $0.67 \pm 0.13$ & $0.79 \pm 0.04$ \\
& $(5.63 \%)$ & $(15.19 \%)$ & Expt. $^{[125]}$ \\
\hline $\mathrm{nC}_{6}-\mathrm{nC}_{12}$ & $1.19 \pm 0.13$ & $1.12 \pm 0.10$ & $1.06 \pm 0.07$ \\
& $(11.16 \%)$ & $(5.66 \%)$ & Expt. $^{[125]}$ \\
\hline
\end{tabular}

a,b The error bars in all methods are due to repeatability.

In evaluating the proposed algorithm further, the effect of employing the algorithm to study systems of different sizes was considered. The outcome of this investigation is summarized in Figure 4. 5. It is evident that for smaller systems with fewer particles (molecules), there are much larger deviations from the experimental data. As we move towards larger systems, there is a more stable performance of the algorithm, with the results containing smaller errors and matching closely with the experimental data. For instance for equimolar mixture of $\mathrm{nC}_{5}-\mathrm{nC}_{10}$ in a small system with only 400 molecules mHEX overestimated the experimental with $39.75 \%$ while for the largest system with 3200 molecules the relative error is reduced to $4.16 \%$. Collectively, these results present a strong case for using moderately large systems to investigate problems pertaining to heat conduction and thermodiffusive flows. 


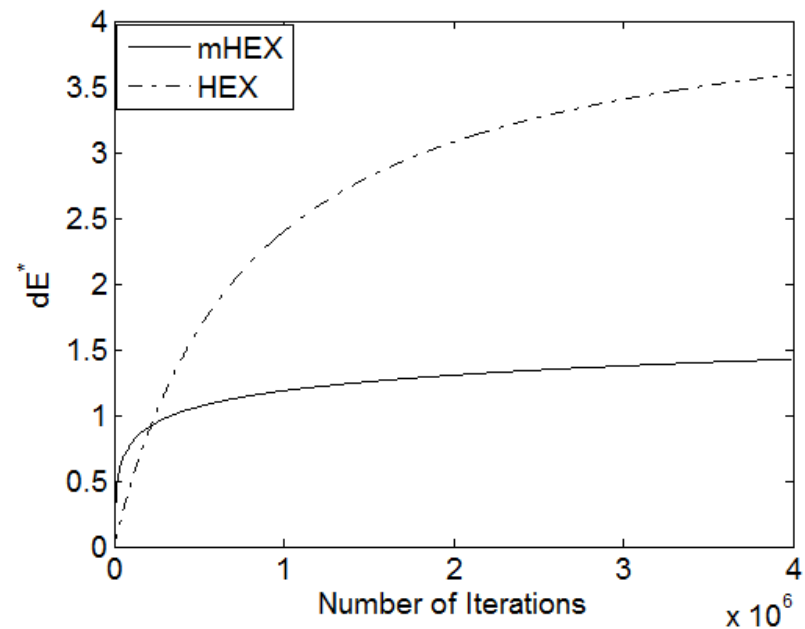

Fig. 4.3: Magnitude of cumulative energy loss for the $\mathrm{MD}$ simulation of the $\mathrm{nC}_{5}-\mathrm{nC}_{10}$ mixture with mole fraction of $\mathrm{nC}_{5}=0.2$ from the $\mathrm{mHEX}$ and $\mathrm{HEX}$ algorithms.

While large systems with many particles are desirable, the computational power needed to make such calculations can be a limiting factor. Everything else remaining fixed, the computational efficiency of the algorithm plays an important role in dictating the size of the system. The computational efficiency of mHEX is quantified by comparing the computational times of the two algorithms with each other when the serial implementation of the two algorithms are used to investigate the mixtures on the same cpu. Figure 4. 6 summarizes the savings in computational time by switching to mHEX algorithm. As seen in this figure, the computational time is a nonlinear function of the size of the system, and the disparity in the computational time needed by the two algorithms increase with the number of particles in the system, favouring the mHEX algorithm. As expected, for the smaller systems the savings in computational time are smaller. However, as the size of the system increases, there is an average saving of about $9 \%$ when the system size is at 3200 particles. 

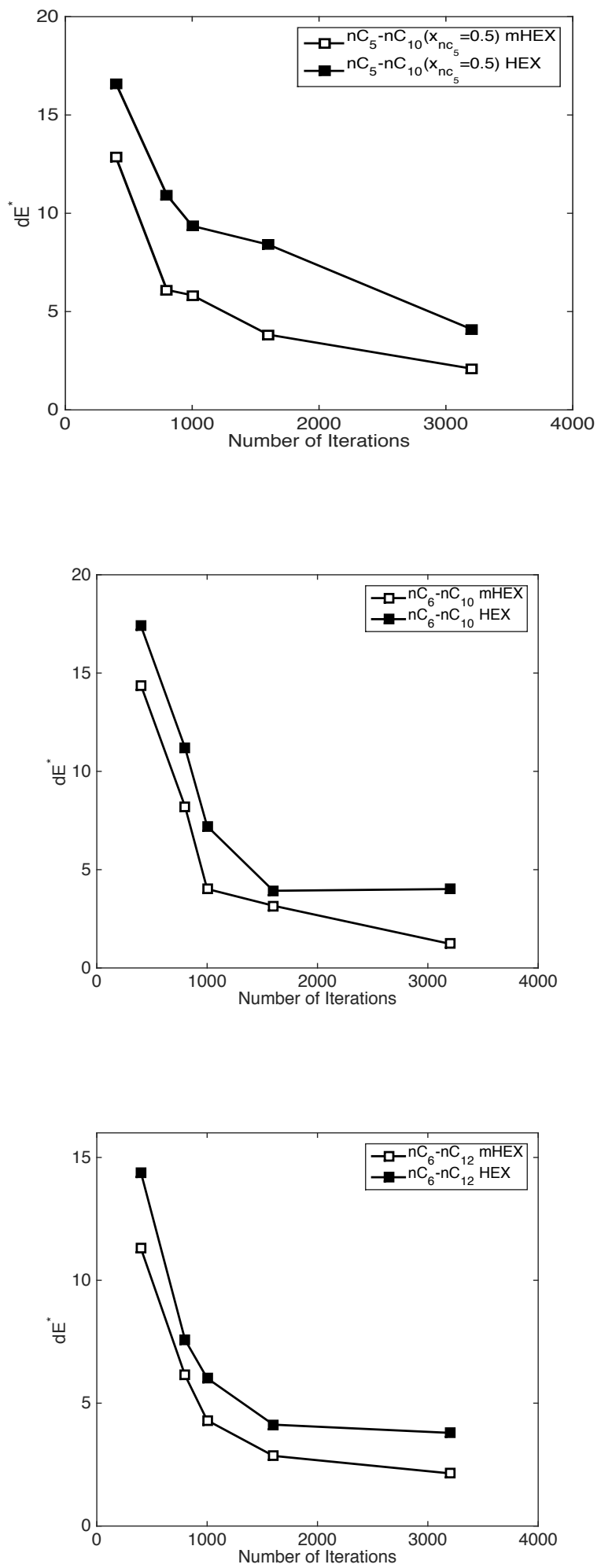

Fig. 4.4: Magnitude of the total energy loss at the end of the simualtion as a function of number of particles. 

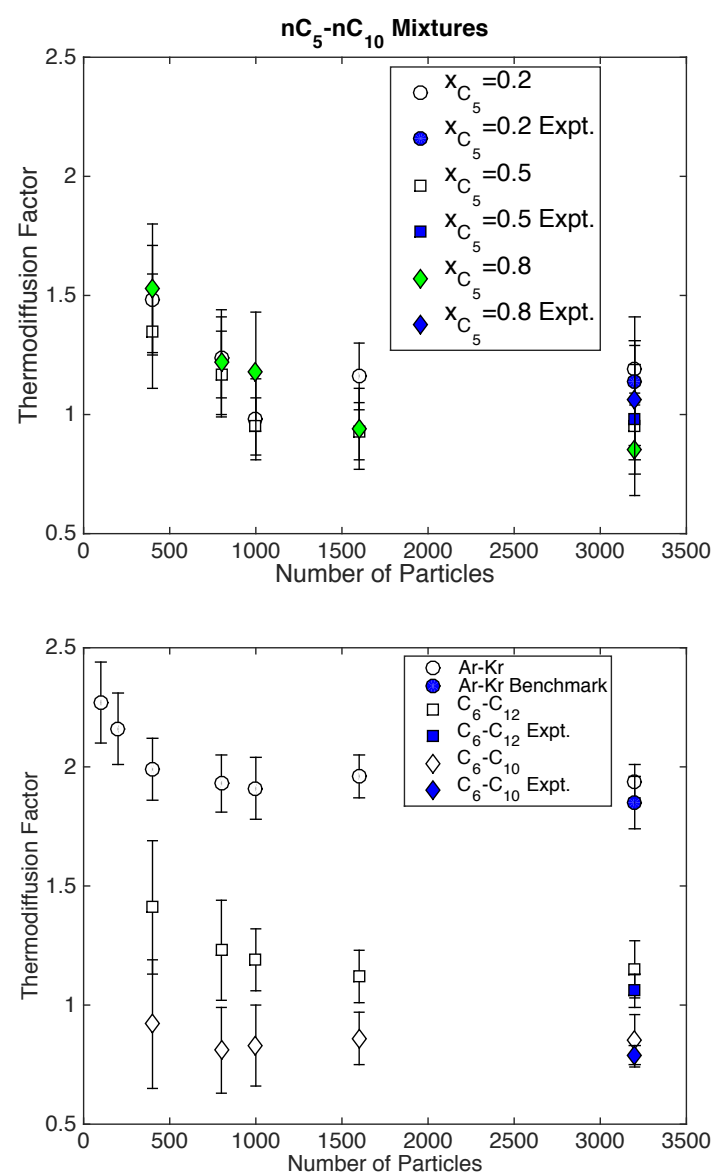

Fig. 4.5: Thermodiffusion factor calculated using mHEX algorithm as a function of the number of particles in the system.

\subsection{Summary \& Conclusions}

In this work we propose a modified form of HEX algorithm to conduct molecular dynamics simulations of liquid mixtures subject to thermal gradients. The main application areas include isotope separation, biomolecular binding curves, trapping of DNA, thermal field flow fractionation devices for polymer characterization, fluid transport in outer space and freeze drying of food. This scientific computation is also relevant in natural processes such as salinity of ocean, solar ponds and crude oil stratification in underground oil reservoirs. 

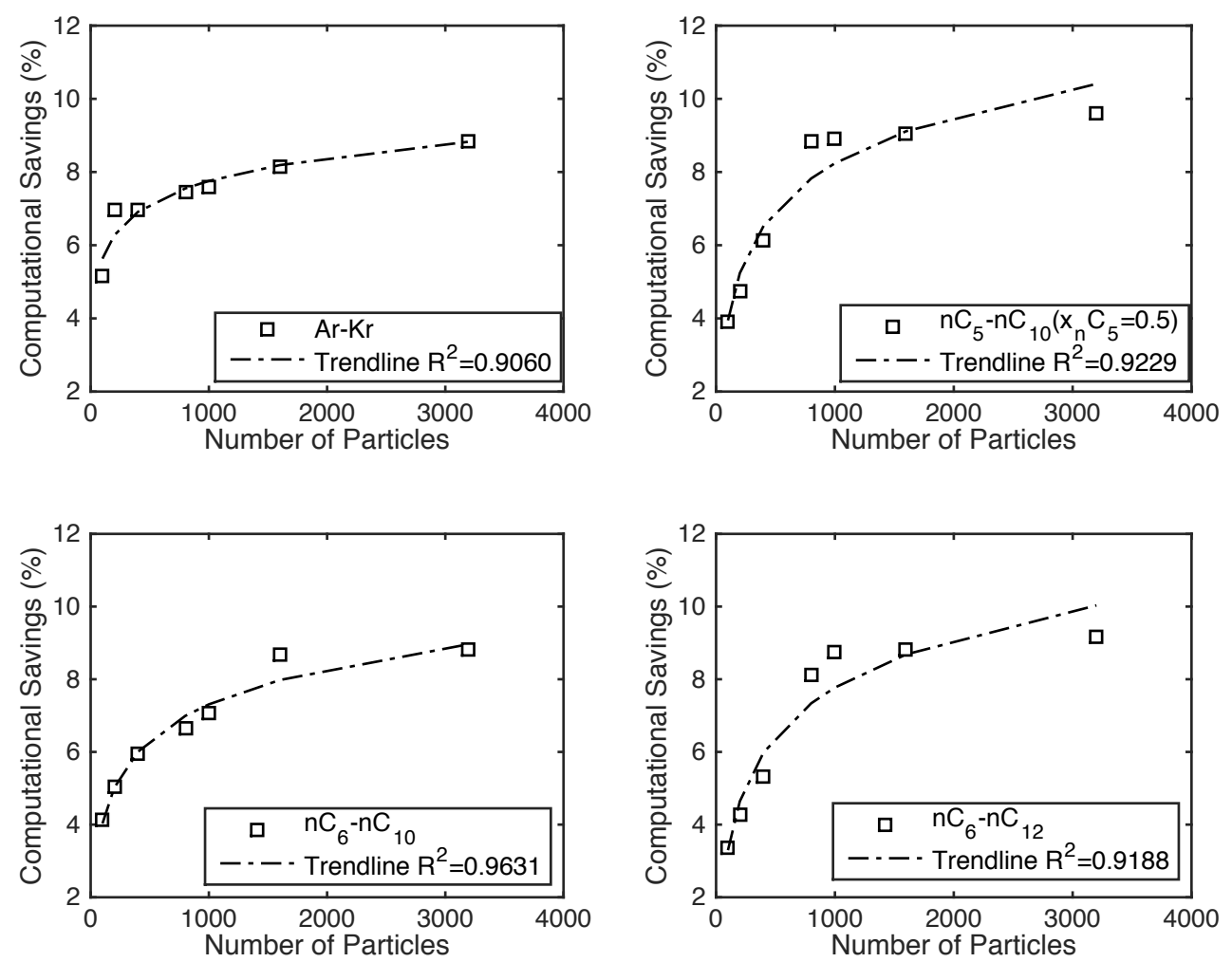

Fig. 4.6: Savings in the computational time as a function of the size of the system. Dotted line indicates the logarithmic trend.

The modification includes eliminating a $\mathrm{O}\left(\mathrm{N}_{\mathrm{p}}\right)$ calculation of the rescaling factor in the velocity rescaling equation, from inside the main time loop of the HEX algorithm. This is replaced by Equation (2), based on the composition and thermodynamic properties of the mixture, that is calculated only once at the beginning of the simulation, outside the main time loop.

The modified algorithm (mHEX) has been applied to six different binary mixtures to study the diffusion of the species in response to a temperature gradient. From the results of 42 molecular dynamics simulation cases, each repeated 4 times, it has been found that:

(1) The uniform value of in mHEX means that there are no arbitrary fluctuations of velocity of particles when applying velocity rescaling in the domain, thereby resulting in more accurate calculations, closely matching 
the experimental data. The mHEX algorithm overestimated the thermodiffusion factor with average relative error $37 \%$ for the smallest systems with 400 molecules. While the average relative error for largest system with 3200 molecules was reduced to $7 \%$.

(2) The mHEX algorithm subdued the energy loss by $20 \%$ for small systems with 400 particles, while for large system consisting of 1600 and 3200 particles the improvement was $44 \%$.

(3) Finally, by eliminating the calculation of inside the main time loop and replacing it by a fixed value determined by Eqn. (4.2) outside the main time loop, there is a $\mathrm{O}\left(\mathrm{N}_{\mathrm{p}}\right)$ savings in computations. More precisely, an overall savings of about $9 \%$ in computational time has been observed by employing mHEX algorithm proposed in this work. 


\section{Chapter 5- Conclusions \& Future Work}

\subsection{Conclusions}

Given the importance of thermodiffusion in oil industry, several researchers investigated thermodiffusive segregation in hydrocarbon mixtures in macro scale in both theoretical and experimental fronts. The major objective of these studies was to predict the strength and sign of thermodiffusion via parameter called thermodiffusion factor. The theoretical models often contradict each other while the experimental methods are prone to various sources of errors including unwanted vibrations and natural gravity fields. As a result, the main objective of this research is to study thermodiffusive flows through consideration of molecular interactions between different type particles as well as estimation the Soret coefficient for binary and ternary hydrocarbon mixtures via molecular dynamics simulations. In doing so, a computationally efficient and accurate algorithm has been developed.

The new algorithm proposed in this thesis has been exhaustively evaluated with respect to 20 binary and ternary liquid mixtures. More precisely, three different types of binary hydrocarbon mixtures, namely, $\mathrm{nC}_{6}-\mathrm{nC}_{10}, \mathrm{nC}_{6}-\mathrm{nC}_{12}$ and $\mathrm{nC}_{5}-\mathrm{nC}_{10}$ were studied. The first two mixtures were studied for six different compositions while only three compositions were considered for the last mixture. Additionally, during validation process the numerical results for one non-equimolar mixture of $\mathrm{nC}_{6}-\mathrm{nC}_{12}$ and $\mathrm{nC}_{6}-\mathrm{nC}_{10}$, three nonequimolar mixture of $\mathrm{nC}_{5}-\mathrm{nC}_{10}$ were compared with available experimental data in the literature. In case of ternary mixtures, three different compositions of normal alkanes of $\mathrm{nC}_{1}-\mathrm{nC}_{4}-\mathrm{nC}_{12}$ as well as one composition of different types alkane mixtures of $\mathrm{nC}_{12}$-IBBTHN were validated with respect to numerical and experimental results in the literature, respectively.

In the MD simulations, hydrocarbon mixtures were modelled as a N-body particle system within a cubic simulation domain. The intermolecular interactions were modelled by Lennard-Jones pair potential with a cut of ratio. The Lorentz-Berthelot rules were applied 
to obtain the atomic parameters of unlike particles in the system. The Verlet-velocity integration method were used to update the particles' velocities and at every time step. Additionally, periodic boundary conditions as well as minimum image convection were applied across directions to curb the wall impacts.

First, two popular well-known algorithms in literature, i.e. RNEMD and HEX, were adopted for heat generation process in the system. The primary application of these algorithms was to calculate thermal conductivity in the system; however, they have been used to study the thermodiffusive flows as well. Given the dearth of comprehensive and detailed reviews in literature, the performance of these two algorithms for different binary mixtures including mixture of $\mathrm{Ar}-\mathrm{Kr}$ as well as hydrocarbon mixture of $\mathrm{nC}_{6}-\mathrm{nC}_{10}$ were compared with respect to experimental and numerical results in the literature. The HEX algorithm demonstrated marginal superiority over the RNEMD.

Second, after close observation of HEX algorithm, a new modified version of HEX, viz. mHEX, was presented in this research for the first time. The behaviour of the new modified version in predicting Soret coefficients for several binary and ternary mixtures was validated with respect to experimental data in literature. With respect to the regular HEX algorithm, the mHEX algorithm has shown significant improvements in accuracy of estimation of the Soret coefficients with respect to experimental data as well as savings in the computational time. While the principle of heat generation in all the algorithms involves the manipulation of particles' velocity at certain location in the simulation domain, each algorithm has a unique methodology to rescale the velocities without violation of conservation of momentum. However, the algorithm proposed in this thesis is the most accurate and efficient.

\subsection{Contributions}

The major contributions of this dissertation based on the results and conclusions presented in pervious chapters are as follows: 
- A modified version of widely used boundary driven HEX algorithm, i.e. mHEX, was introduced to study Soret effect in binary and ternary mixtures in molecular level.

- The scaling factor in mHEX algorithm is calculated once at the beginning of the simulation as a function of mixtures properties. This methodology to calculate the scaling factor saved computational time for small systems $3-4 \%$ and $8-9 \%$ for large systems. This is an $\mathrm{O}\left(\mathrm{N}^{2}\right)$ savings in computational time, $\mathrm{N}$ being the number of particles in the system.

- The energy drift in the new proposed algorithm has been curbed in early stage of simulation and the magnitude of accumulative energy loss decreased by nearly $30 \%$ from the regular HEX algorithm that is usually used in the literature.

- In general, the new algorithm has improved the prediction of thermodiffusion factor in binary mixture by $24 \%$ in comparison with regular HEX algorithm. Additionally, the results of mHEX algorithm for estimating the strength of thermodiffusive segregation in ternary mixture was $17 \%$ more accurate than traditional HEX algorithm. It must be mentioned that the experimental data were obtained in microgravity environment.

\subsection{Future Work}

The following research areas are recommended for the future works:

- Studying thermodiffusive flow in a multi-scale structure of an oil reservoir by integration of the current MD simulation tool. This can help determine the largescale stratification process of crude oil.

- Implementation of more sophisticated intermolecular potential functions by adding more features into current MD simulation tool. This can further enhance accuracy. However, this can lead to slowing down of the algorithm, so one must tread carefully if speed is an important criteria.

- Studying and investigating the Soret effect for quaternary hydrocarbon mixtures using mHEX algorithm. 
- Validating the mHEX algorithm for different types of mixtures including polymers and associating mixtures. This can enable us to apply the algorithm to study other applications. 


\section{Appendices}

\section{Appendix A}

\section{A.1 General Overview Molecular Dynamics}

Molecular dynamics (MD) is a powerful computational tool to study physical movements of particles. It must be noted that in this thesis we use the word particles and molecules interchangeably since we do not consider the intra-molecular effects such as the size, shape, bond angles etc. in our simulations. Instead we look at the entire molecule as a single "particle". Where, the constituents of $\mathrm{N}$-body system are allowed to interact with each other. The application of molecular dynamics (MD) in estimation of equilibrium and dynamic properties of simple fluid systems as well as complicated fluid mixtures has demonstrated a noticeable success in various research areas including biology, material and thermofluids. The MD techniques can be classified into two main categories: equilibrium methods $[110,111]$ and non-equilibrium approaches including boundary driven and synthetic NEMD $[112,113,159]$. In the former method the transport properties can be calculated through Green-Kubo or Einstein formula, which links the integral of auto-correlation of flow quantities to corresponding dynamic properties in the absence of any agitating field. The later technique computes the dynamic properties of the system in the present of external forces or perturbing field.

\section{A.2 Potential Function}

The most significant and tedious part of modelling an N-particle system in molecular level is simulation of constituents' interaction. In general, quantum mechanics describes the interaction of simple and complicated molecular structure; however; MD methods by adopting its classical viewpoint, assume that molecules are massive point objects and interactions between these points can be explained through pair potential functions that depend upon the distance of separations of these points [122]. 
Various potential functions have been proposed for different application range of intermolecular interactions [122]. Lennard-Jones (LJ) pair potential function is the simplest suggested potential function with strong repulsive core and weak attractive tail, Eq. (1.1). This simple pair potential function has proved to be a suitable choice for hydrocarbon mixtures [84, 86]. Moreover, it is less time-consuming and often outperforms the more complicated models [86].

$\left.\phi_{i}=\phi\left(r_{i j}\right)=4 \varepsilon_{i j}\left(\frac{\sigma_{i j}}{r_{i j}}\right)^{12}-\left(\frac{\sigma_{i j}}{r_{i j}}\right)^{6}\right]$

where, $\phi_{i} r_{i j}, \varepsilon_{i j}$ and $\sigma_{i j}$ represent pair potential (J), distance between the particles (m), well-depth potential $(\mathrm{J})$ and atomic diameters $(\mathrm{m})$, respectively. Also, subscripts $\mathrm{i}, \mathrm{j}$ indicate unlike particles.

Since the major simulation time spends on calculation of these potentials and their corresponding forces, cut off ratio distance $\left(r_{c}\right)$ technique can be used to reduce the computational time. When the separation distance between particles are greater than cut of ratio, the potential and its related force are set to zero. Different methods can be used to calculate the atomic diameter as well as potential strength for dissimilar particles. However, Lorentz-Berthelot rules have been widely used for hydrocarbon mixtures [80, 84-86], Eq. (A.2) and Eq. (A.3).

$\sigma_{i j}=0.5\left(1-l_{i j}\right)\left(\sigma_{i i}+\sigma_{i j}\right)$

$\varepsilon_{i j}=\left(1-k_{i j}\right) \sqrt{\left(\varepsilon_{i} \varepsilon_{j}\right)}$

In the above equations $l_{i j}$ and $k_{i j}$ are cross-interaction parameters and for simple alkane molecules are negligible. Besides, the intermolecular force on each particle is equal to the negative sign of gradient of pair potential, i. e. $\vec{F}_{i j}=-\vec{\nabla} \phi_{i j}$. 
These forces are only function of particle's separation distance and intermolecular parameters and dictate the amount of accelerations on each particle. At every time step, particles positions are updated based on obtained accelerations and previous position and velocity of each particle.

\section{A.3 Integration Methods}

In order to achieve a reliable average macroscopic property of a microscopic $\mathrm{N}$-particle system, three basic steps must be implemented properly. First, an acceptable potential function to represent the intermolecular interaction must be selected. Second, the calculation of forces based on the elected potential model in preceding stage. Finally, an effective algorithm for integration of equation of motion is required. The essence of most common numerical integration technique is implementation of Taylor series. Verlet and Gear's predictor- corrector algorithms are the most common integration methods [122]. However, Verlet algorithm outperforms the Gear's predictor-corrector technique in terms of energy drift [86].

$$
\begin{aligned}
& \vec{r}_{i}(t+\Delta t)=\vec{r}_{i}(t)+\vec{V}_{i}(t) \Delta t+\frac{\Delta t^{2}}{2 m_{i}} \sum_{j, j \neq i} \vec{F}_{i j}\left(\vec{r}_{i}(t), \vec{r}_{j}(t)\right) \\
& \vec{a}_{i}(t+\Delta t)=\frac{1}{m_{i}} \sum_{j, j \neq i} \vec{F}_{i j}\left(\vec{r}_{i}(t+\Delta t), \vec{r}_{j}(t+\Delta t)\right) \\
& \vec{V}_{i}(t+\Delta t)=\vec{V}_{i}(t)+\frac{\Delta t}{2}\left(\vec{a}_{i}(t)+\vec{a}_{i}(t+\Delta t)\right)
\end{aligned}
$$

where, $t, V, m$ and $a$ are time (s), velocity $\left(\mathrm{m} \cdot \mathrm{s}^{-1}\right)$, mass $(\mathrm{kg})$ and acceleration $\left(\mathrm{m} \cdot \mathrm{s}^{-2}\right)$.

\section{A.4 Periodic Boundary Condition}


In general, considerable amount of molecules lie on boundary surfaces in MD models. Simulation can be subjected to the substantial inaccuracy in determination of properties due to the different nature of the forces applied on particles on the boundaries. In most cases, implementation of periodic boundary condition can reduce the errors.

Periodic boundary condition simply can be considered as an infinite, space-filling array of identical copies of simulation region. In another words, a central simulation cell will be replicated through the space to form an infinite lattice. As a result, when a particle leaves the simulation region through a particular bounding face immediately re-enters another cell through the opposite face. Moreover, the particles' interactions within $r_{c}$ distance will be limited to adjacent cells [122].

\section{A.5 Calculation of Properties}

Monitoring the temperature and energy are crucial for a system that is subjected to the thermal field. The instantaneous temperature of a system can be determined via applying statistical mechanics and Virial theorem. The average temperature of a system can be achieved through time average [122].

$T_{i n s}=\frac{1}{3 N k_{b}} \sum_{i=1}^{N} m_{i} V_{i}^{2}$

Total potential and kinetic can be calculated based on following equations:

$$
\begin{aligned}
& E_{k}=\frac{3}{2} N k_{b}<T_{i n s}> \\
& E_{p}=<\sum_{j=1}^{N} \sum_{i=1}^{N} \phi_{j}>
\end{aligned}
$$


Additionally, the microscopic heat flux can be obtained based on the following formula [122]:

$J_{u}=<\frac{1}{V o l} \sum\left(\left(E_{k}+E_{p}\right)\left(v_{i}-v_{b}\right)-\frac{1}{2} \sum_{i=1}^{N} \mid\left(F_{i j} \cdot\left(v_{i}-v_{b}\right) \mid \cdot r_{i j}\right)>\right.$

In the above equations, $T_{i n s}, N, k_{b}, E_{k}$ and $E_{p}$ denote instantaneous temperature $(\mathrm{K})$, number of particles, Boltzmann constant $\left(\mathrm{J}^{\mathrm{K}} \mathrm{K}^{-1}\right)$, kinetic energy and potential energy, respectively. Also, $>>$ symbol represent mathematical averaging.

Additionally, in MD simulation dimensionless parameters (reduced parameters) are often considered noticeable asset. Some of the essential reduced parameters can be obtained based on following formulas [86].

$\rho^{*}=\frac{N}{V o l} \sigma^{3}$

$T^{*}=\frac{K_{b} T}{\varepsilon}$

$t^{*}=\frac{t}{\sigma} \sqrt{\frac{\varepsilon}{m}}$

$V^{*}=V \sqrt{\frac{m}{\varepsilon}}$

$E^{*}=\frac{E}{\varepsilon}$

$J_{u}^{*}=J_{u} \frac{\sigma^{3}}{\varepsilon} \sqrt{\frac{m}{\varepsilon}}$ 


\section{Appendix B}

Table. B Computational cases used in chapter 4 outlining each mixture, the size of the system and the mole fraction of the first component in the system.

\begin{tabular}{|c|c|c|c|}
\hline Case \# & Mixture & \# Particles & Mole Fraction of component 1 \\
\hline 1 & \multirow{7}{*}{$\mathrm{Ar}-\mathrm{Kr}$} & 100 & \multirow{7}{*}{0.5} \\
\hline 2 & & 200 & \\
\hline 3 & & 400 & \\
\hline 4 & & 800 & \\
\hline 5 & & 1000 & \\
\hline 6 & & 1600 & \\
\hline 7 & & 3200 & \\
\hline 8 & \multirow{7}{*}{$\mathrm{nC}_{5}-\mathrm{nC}_{10}$} & 100 & \multirow{7}{*}{0.2} \\
\hline 9 & & 200 & \\
\hline 10 & & 400 & \\
\hline 11 & & 800 & \\
\hline 12 & & 1000 & \\
\hline 13 & & 1600 & \\
\hline 14 & & 3200 & \\
\hline 15 & \multirow{7}{*}{$\mathrm{nC}_{5}-\mathrm{nC}_{10}$} & 100 & \multirow{7}{*}{0.5} \\
\hline 16 & & 200 & \\
\hline 17 & & 400 & \\
\hline 18 & & 800 & \\
\hline 19 & & 1000 & \\
\hline 20 & & 1600 & \\
\hline 21 & & 3200 & \\
\hline 22 & & 100 & \\
\hline 23 & & 200 & \\
\hline 24 & & 400 & \\
\hline
\end{tabular}




\begin{tabular}{|c|c|c|c|}
\hline 25 & $\mathrm{nC}_{5}-\mathrm{nC}_{10}$ & 800 & 0.8 \\
\hline 26 & & 1000 & \\
\hline 27 & & 1600 & \\
\hline 28 & & 3200 & \\
\hline 29 & \multirow{7}{*}{$\mathrm{nC}_{6}-\mathrm{nC}_{10}$} & 100 & \multirow{7}{*}{0.38} \\
\hline 30 & & 200 & \\
\hline 31 & & 400 & \\
\hline 32 & & 800 & \\
\hline 33 & & 1000 & \\
\hline 34 & & 1600 & \\
\hline 35 & & 3200 & \\
\hline 36 & \multirow{7}{*}{$\mathrm{nC}_{6}-\mathrm{nC}_{12}$} & 100 & \multirow{7}{*}{0.34} \\
\hline 37 & & 200 & \\
\hline 38 & & 400 & \\
\hline 39 & & 800 & \\
\hline 40 & & 1000 & \\
\hline 41 & & 1600 & \\
\hline 42 & & 3200 & \\
\hline
\end{tabular}




\section{Appendix C}

Table. C: Thermodynamic state of each mixture used in Chapter 4.

\begin{tabular}{|c|c|c|c|}
\hline Mixture & $\boldsymbol{T}^{*}$ & $\boldsymbol{\rho}^{*}$ & References \\
\hline $\mathrm{Ar}-\mathrm{Kr}$ & 0.9650 & 0.7137 & {$[78]$} \\
\hline $\mathrm{nC}_{5}-\mathrm{nC}_{10}\left(\mathrm{X}_{\mathrm{nC}_{5}}=0.2\right)$ & 0.6363 & 1.0491 & {$[126]$} \\
\hline $\mathrm{nC}_{5}-\mathrm{nC}_{10}\left(\mathrm{X}_{\mathrm{nC}_{5}}=0.5\right)$ & 0.6363 & 1.2088 & {$[126]$} \\
\hline $\mathrm{nC}_{5}-\mathrm{nC}_{10}\left(\mathrm{X}_{\mathrm{nC}_{5}}=0.8\right)$ & 0.6363 & 1.4212 & {$[126]$} \\
\hline $\mathrm{nC}_{6}-\mathrm{nC}_{10}$ & 0.6123 & 1.2130 & {$[125]$} \\
\hline $\mathrm{nC}_{6}-\mathrm{nC}_{12}$ & 0.5462 & 1.2580 & {$[125]$} \\
\hline
\end{tabular}




\section{References}

[1] Platten, J. K., The Soret Effect: A review of recent experimental result, J Appl Mech, 73 (5) (2006), 5-15.

[2] Soret, C., Sur l'état d'équilibre que prend au point de vue de saconcentration une dissolution saline primitivement homohéne dont deux partiessont portées à des températures différentes, Arch. Sci. Phys. Nat, 2 (1879), 48-61.

[3] Soret, C., Influence de la température sur la distribution des sels dans leurs solutions, Acad. Sci. Paris, 91 (5) (1880), 289-291.

[4] Soret, C., Sur l'état d'équilibre que prend au point de vue de sa concentration une dissolution saline primitivement homohéne dont deux par- ties sont portées à des températures différentes, Ann. Chim. Phys., 22 (1881), 293-297.

[5] Tyndall, J., Scientific addresses, Charles C. Chatfield \& Co., New Haven, 1870.

[6] Omeiri, D., \& Djafri, D. E., Transport properties in gases at high temperature and low pressure: Comparison of kinetic theory with direct simulation Monte Carlo, Int. J. Thermophys., 31 (6) (2010), 1111-1130.

[7] Bidabadi, M., Mostafavi, S. A., \& Asadollahzadeh, P., Nonlinearity effect of transport properties on combustion of volatile organic particle, Heat Transf. Res., 43 (3) (2012), 237-257.

[8] Takeyama, N., \& Nakashima, K., Thermodynamics of thermal diffusion in aqueous ionic solutions, J. Phys. Soc. Jpn., 52 (8) (1983), 2699-2705. 
[9] Zhao, D., Wang, H., Khan, Z. U., Chen, J. C., Gabrielsson, R., Jonsson, M. P., Berggren, M., \& Crispin, X., Ionic thermoelectric supercapacitors, Energy Environ. Sci., 9 (4) (2016), 1450-1457.

[10] Coteseque, P., Gaillard, S., Gachet, Y., \& Jamet, Ph., Determination of the Apparent negative Soret coefficient of water- $10 \%$ alcohol solutions by experimental and numerical methods in packed cells, Philos. Mag., 83 (17-18) (2003), 2039-2044.

[11] Shashkov, A. G., Zolotukhina, A. F., Vasilenko, V. B., \& Kolenchits, O. A., Factor of thermodiffusion of binary mixtures containing alcohol vapour, Eng. Phys. Thermophys., 78 (3) (2005).

[12] Eslamian, M., \& Saghir, M. Z., Thermodiffusion in binary and ternary nonpolar hydrocarbon+alcohol mixtures, J. Non-Equil. Thermody., 37 (4) (2012), 329-351.

[13] Wiegand, S., Thermal Diffusion in liquid mixtures and polymer solutions, J. Phys.: Condensed Matter, 16 (10 (2004), R357-R379.

[14] Bringuier, E., Thermophoresis of linear polymer chains, Comptes Rendus Mécanique, 339 (5) (2011), 349-354.

[15] Mingcheng, Y., \& Ripoll, M., Driving forces and polymer hydrodynamics in the soret effect, J. Phys.: Condensed Matter, 24 (19) (2012),195101 (6 Pages).

[16] Jafar-Salehi, E., Eslamian, M., \& Saghir, M. Z., Effect of thermodiffusion on the fluid flow, heat transfer, and solidification of molten metal alloys, Engineering Science and Technology an International Journal, 19 (1) (2016), 511-517.

[17] Blums, E., S. Odenbach, Mezulis, A., \& Maiorov, M., Soret coefficient of nanoparticles in ferrofluids in the presence of a magnetic field, Phys. Fluids, 10 (9) (1998), 2155-2163. 
[18] Volker, T., \& Odenbach, S., Thermodiffusion in magnetic fluids, J. Magn. Magn. Mater., 289 (2) (2005), 289-291.

[19] Sprenger, L., Lange, A., \& Odenbach, S., Thermodiffusion in concentrated ferrofluids: Experimental and numerical on magnetic, Phys. Fluid, 26 (2) (2014), 0022001-1-16.

[20] Mahler, G., Maier, G., Forchel, A., Laurich, B., Sanwald, H., \& Schmid, W., Thermodiffusion of high-density electron-hole plasmas in semiconductors, Phys. Rev. Lett., 47 (25) (1981), 1855-1858.

[21] Williams, R. K., \& Philbrook, The Soret effect in molten Ag-Te Solurions, J. Electrochemical Society, 128 (5) (1981), 1034-1040.

[22] Eslamian, M., \&Saghir, Z. M., Thermodiffusion (thermomigration) and convection in molten semiconductor-metal layers, Int. J. Therm. Sci., 50 (70) (2011), 1232-1242.

[23] Putnam, S. A., Transport of nanoscale latex spheres in a temperature gradient, Langmuir, 21 (12) (2005), 5317-5323.

[24] Hutson, M. S., Hauger, S. A., \& Edwards, G., Thermal diffusion and chemical kinetics in laminar biomaterial due to heating by a free-electron laser, Phys. Rev. E, 65 (6) (2002), 06190-1-6.

[25] Gadmoski, A., \& Kruszewska, N., Thermodiffusion as a close-to-interface effect that matters in non-isothermal (dis)orderly protein aggregations, Phys. Lett. A., 378 (38) (2014), 2881-2887.

[26] Costeséque, P., Mojtabi, A., \& Platten, J. k., Thermodiffusion phenomena, Comptes Rendus Mécanique, 339 (5) (2011), 275-279. 
[27] Kim, S. J. \& Stossel, A., Impact of subgrid-scale convection on global thermohaline properties and circulation, J. of Phys. Oceanography 31 (3) (2001), 656-674.

[28] Hughes, D. W., \& Weiss, N. O., Double-diffusive convection with two stabilizing gradients: strange consequences of magnetic buoyancy, J. Fluid Mech., 301 (1) (1995), 383-406.

[29] Kempers, L. M., A thermodynamic theory of the Soret effect in a multicomponent liquid, J. Chem. Phys., 90(11) (1989), 6541-6548.

[30] Rutherford, W.M., Dougherty, E. L., \& Drickamer, H. G., Thermal diffusion in binary mixtures $\mathrm{CS}_{2}$ and hexane isomers, J. Chem. Phys., 22 (8) (1954), 1289-1292.

[31] Dougherty, E. L., \& Drickamer, H. G., A theory of thermal diffusion in liquids, J. Chem. Phys., 23 (2) (1955), 295-309.

[32] Dougherty, E. L., \& Drickamer, H. G., Thermal diffusion and molecular motion in liquids, J. Phys. Chem., 59 (5) (1955), 433-449.

[33] Emery, A. H., \& Drickamer, H. G., Thermal diffusion in polymer soultions, J. Chem. Phys., (23) (12) (1955), 2252-2257.

[34] Winter, F. R., \& Drickamer, H. G., thermal diffusion in single crystal of zinc, J. Chem. Phys., 24 (2) (1956), 492-493.

[35] Haase, R., Thermodynamics of Irreversible Processes, Addison-Wesley, Boston, USA, 1969.

[36] Mortimer, R. \& Eyring, H., Elementary transition state theory of Soret and Dufour effects, Natal. Acad. Sci., 77 (4) (1980), 1728-1731. 
[37] Guy, A. G., Prediction of thermal diffusion in binary mixtures of nonelectrolyte liquids by the use of non-equilibrium thermodynamics, Int. J. Thermophys., 7(3) (1986), 563-572.

[38] Kempers, L. M., A comprehensive thermodynamic theory of the Soret effect in a multicomponent gas liquid, or solid, J. Chem. Phys., 115 (14) (2001), 6330-6341.

[39] Brenner, H., Is the tracer velocity of a fluid continuum equal to its mass velocity?, Phys. Rev. E., 70 (6) (2004), 061201-1-10.

[40] Brenner, H., \& Bielenberg, J. R., A continuum approach to phoretic motions: Thermophoresis, Physica A: Statistical mechanics and Its Applications, 355(2-4) (2005), 251-273.

[41] Bielenberg, J. R., \& Brenner, H., A continuum model of thermal transpiration, J. Fluid Mechanics, 546 (1) (2006), 1-23.

[42] Brenner, H., Navier-Stokes revisited, Physica A: Statistical mechanics and Its Applications, 349 (1-2) (2005), 60-132.

[43] Eslamian, M., \& Saghir, M. Z., Microscopic study and modeling of thermodiffusion in binary associating mixtures, Phys. Rev. E, 80 (6) (2009), 061201-1-12.

[44] Eslamian, M., \& Saghir, M. Z., A dynamic thermodiffusion model for binary liquid mixtures, Phys. Rev. E, 80 (1) (2009), 011201-1-11.

[45] Eslamian, M., \& Saghir, M. Z., Modeling of DNA thermophoresis in dilute solutions using the non-equilibrium thermodynamics approach, J. Non-Equil. Thermody., 37 (1) (2012), 63-76.

[46] Eslamian, M., Saghir, M. Z., Non-equilibrium thermodynamic model for the estimation of the Soret coefficient in dilute polymer solutions, Int. J. Thermophysics, 32 (3) (2011), 652-664. 
[47] Abbasi, A., Saghir, M. Z., \& Kawaji, M., An improved thermodiffusion model for ternary mixtures using Fujita's free volume, J. Non-Equil. Thermody., 36 (3) (2011), 259-272.

[48] Eslamian, M., Saghir, M., Z., \& Bou-Ali, M. M., Investigation of the Soret effect in binary, ternary and quaternary hydrocarbon mixtures: a new expressions for thermodiffusion factors in quaternary mixtures, Int. J. Therm. Sci., 49 (11) (2010), 21282137.

[49] Eslamian, M., \& Saghir, M. Z., A critical review of thermodiffusion models: Role and significant of the heat transfer and activation energy of viscous flow, J. Non-Equil. Thermody., 34 (2) (2009), 97-131.

[50] Rahman, M. A., \& Saghir, M. Z., Thermodiffusion or Soret effect: Historical review, Int. J. Heat Mass Transf., 73 (2014), 693-705.

[51] Butler, B. D., \& Turner, J. C. R., Flow-cell studies of thermal diffusion in liquids. Part 1. -Cell construction and calibration, Trans. Faraday Soc, 62 (1966), 3114-3120.

[52] Clusius, K., \& Dickel, G., Das trennrohrverfarhren bei flüssigkeiten, Naturwissenschaften, 27 (9) (1939), 148-149.

[53] Dutrieux, J. F., Platten, J. K., Chavepeyer, G., \& Bou-Ali, M. M., On the measurements of positive Soret coefficients, J. Phys. Chem., 106 (23) (2002), 6104-6114.

[54] Königer, A., Meier, B., \& Köhler, W., Measurement of the Soret, diffusion, and thermal diffusion coefficients of three binary organic benchmark mixtures and of ethanolwater mixtures using a beam deflection technique, Philos. Mag., 89 (10) (2009), 907-923.

[55] Wiegand, S., Ning, H., \& Kriegs, H., Thermal diffusion forced Rayleigh scattering setup optimized for aqueous mixtures, J. Phys. Chem. B, 111(51) (2007), 14169-14174.

[56] Duhr, S., \& Braun, D., Why molecules move along a temperature gradient, Proc. Natl. Acad. Sci. U. S. A., 103 (52) (2006), 19678-19682. 
[57] Alves, S., Bourdon, A., \& Neto, A. M. F., Generalization of the thermal lens model formalism to account for thermodiffusion in a single-beam Z- Scan experiment: determination of the Soret Coefficient, J. Opt. Soc. Am, 20 (4) (2003), 713-718.

[58] Taylor, W. L., \& Pickett, P., Noble gas- carbon dioxide thermal diffusion factors: Anomalous behaviour for $\mathrm{Ar} / \mathrm{CO}_{2}$, Int. J. Thermophysics, 7 (4) (1986), 837-849.

[59] Pasti, L., Agnolet, S, \& Dondi, F., Thermal field-flow fractionation of charged submicrometer particles in aqueous media, Anal. Chem., 79 (14) (2007), 5284-5296.

[60] Srinivasan, S., \& Saghir, M. Z., Experimental approaches to study thermodiffusionA review, Int. J. Them. Sci., 50 (7) (2011), 1125-1137.

[61] Srinivasan, S., \& Saghir, M. Z., Significance of equation of state and viscosity on the thermodiffusion coefficients of a ternary hydrocarbon mixture, J. High TemperaturesHigh Pressures, 39 (1) (2010), 65-81.

[62] Mousavi, S. A., Yousefi, T., \& Saghir, Z., effect of gravity orientation in the study of thermodiffusion, Can. Chem. Eng., 95 (2) (2017), 398-408.

[63] Srinivasan, S., \& Saghir, M. Z., Computational evaluation of micro-scale and macroscale error sources in thermodiffusive cell, J. Comput. Sci., 5 (5) (2014), 767-776.

[64] Shevtsova, V., Mialdun, A., Menlikov, D., Ryxhkov, I., Gaponenko, Y., Saghir, Z., Lyumbimova, T., \& Legros, J. C., The IVIDIL experiment onboard the ISS: Thermodiffusion in the presence of controlled vibrations, Comptes Rendus Mecanique, 339 (5) (2011), 310-317.

[65] Gaponenko, Y., Mialdun, A., \& Shevtsova, V., Experimental and numerical analysis of mass transfer in a binary mixture with Soret effect in the presence of weak convection, Eur. Phys. J. E, 37 (10) (2014), 90-99. 
[67] Mialdun, A., Minetti, C., Gaponenko, Y., Shevtsova, V., \& Dubois, F., Analysis of thermal performance of SODI instrument for DCMIX configuration, Microgravity Sci., 25 (1) (2013), 83-94.

[68] Kianian, A., Ahadi, A., \& Saghir, M. Z., Experimental evidence of low Rayleigh vibration on mixture during thermodiffusion experiment, Can. J. Chem. Eng., 91 (9) (2013), 1568-1574.

[69] Srinivasan, S., \& Saghir, M. Z., A neurocomputing model to calculate the thermosolutal diffusion in liquid hydrocarbon mixtures, Neural Comput. Appl., 24 (2) (2014), 287-299.

[70] MacGowan D., \& Evans D. J., Heat and mass transfer in binary liquid mixtures, Phys. Rev. A, 34 (3) (1986), 2133-2141.

[71] Evans, D. J., Non-equilibrium molecular dynamics via Gauss's principle of least constraint, Phys. Rev. A., 28 (2) (1983), 1016-1021.

[72] Evans, D. J., Homogeneous NEMD algorithm for thermal conductivity- application of non- canonical linear response theory, Phys. Lett. A, 19 (9) (1982), pp. 457-460.

[73] HafskJold B., Ikeshoji T., \& Ratkje S. K., On the molecular mechanism of thermal diffusion in liquids, Mol. Phys., 80(6) (1993), 1389-1412.

[74] Ikeshoji T, \& HafskJold, B., Non-equilibrium molecular dynamics calculation of heat conduction liquid and through liquid-gas interface, Mol. Phys., 81 (2) (1994), 251261.

[75] Müller-Plathe F., A simple non-equilibrium molecular dynamics method for calculating the thermal conductivity, J. Chem. Phys., 106 (14) (1997), 6082-6085. 
[76] Polyakov, P., Müller, F., \& Wiegand, S., reverse nonequilibrium molecular dynamics calculation of the Soret coefficient in liquid heptane / benzene mixtures, J. Phys. Chem. B, 112 (47) (2008), 1499-15004.

[77] Furtado, F. A., Silveira, A. J., Abreu, C. A, \& Tavares, F. W., Non-equilibrium molecular dynamics used to obtain Soret coefficients of binary hydrocarbon mixtures, Braz. J. Chem. Eng., 32 (3) (2015), 683-698.

[78] Mozaffari, S. H., Srinivasan, S. \& Saghir, M. Z., Evaluations of molecular dynamics methods for thermodiffusion in binary mixtures, ASME J. Therm. Sci. Eng. Appl., 9 (3) (2017), 031011-1-9.

[79] Yeganegi, S., \& Zolfaghari, M., Non-equilibrium molecular dynamics calculation of thermal diffusion factor in binary mixtures of hard spheres, Fluid Phase Equilibria, 243 (1-2) (2006), 161-165.

[80] Galliero, G., Bugel, M., Duguay, B., \& Montel, F., Mass effect on thermodiffusion using molecular dynamics, J. Non-Equil. Thermody., 32 (3) (2007), 251-258.

[81] Artola, P. A., \& Rousseau, B., Isotopic Soret effect in ternary mixtures: Theoretical predictions and molecular simulations, J. Chem. Phys., 143 (17) (2015), 174503-1-9.

[82] I. Inzoli, J. M. Simon, D. Bedeaux \& S. Kjelstrup, Thermal diffusion and partial molar enthalpy variation of n-butane in silicalite-1, J. Phys. Chem. B, 112 (47) (2008), 14937-14951.

[83] Colombani, J., Galliero, G., Duguay, B., Caltagirone, J. P., Montel, F., \& Bopp, P. A., Molecular dynamics study of thermal diffusion in a binary mixture of alkanes trapped in a slit pore, Philos. Mag., 83 (17-18) (2003), 2087-2095.

[84] Galliero, G., Colombani, J., Bopp, P. A., Duguay, B., Caltagirone, J. P., Montel, F., \& Bopp, P. A., Thermal diffusion in micropores by molecular dynamics computer simulations, Physica A, 361 (2) (2006), 494-510. 
[85] Galliero, G., Srinivasan, \& Saghir, M. Z., Estimation of thermodiffusion in ternary alkane mixtures using molecular dynamics simulations and an irreversible thermodynamic theory, High Temperature- High Pressure, 38 (2010)-315-328.

[86] Galliero, G., Dugyay, B., Caltagirone, J. P., and Montel, F., On thermal diffusion in binary and ternary Lennard-Jones mixtures by non-equilibrium molecular dynamics Philos. Mag., 83 (18) (2003), 2097-2108.

[87] Bresme, F., Hafskjold, B., \& Wold, I., Non-equilibrium molecular dynamics study of heat conduction in ionic systems, J. Phys. Chem., 100 (5) (1996), 1879-1888.

[88] Wirnsberger, P., Frenkel, D., \& Dellago, C., An enhanced version of the heat exchange algorithm with excellent energy conservation properties, J. Chem. Phys., 143 (12) (2015), 124104-1-8.

[89] Srinivasan, S., \& Saghir, Thermodiffusion in Multicomponent Mixtures, Springer, 2013.

[90] Huang, F., Chakraborty, P., Lundstrom, C. C., Holmden, C., Glessner, J. G., Kieffer, S. W., \& Lesher, C. E. , Isotope fractionation in silicate melts by thermal diffusion, Nature 464 (7341) (2010), 396-400.

[91] Halder, A., Dhall, A., and Datta, A. K., Modeling transport in porous media with phase change: applications to food processing, ASME J. Heat Transf., 133 (3) (2011), 031010-1-13.

[92] Würger, A., Molecular-weight dependent thermal diffusion in dilute polymer solutions, Phys. Rev. Lett., (102) 7 (2009), 1-4. 
[93] You, Y., A global ocean climatological atlas of the turner angle: implications for double-diffusion and water-mass structure, Deep-Sea Research Part I: Oceanographic Research Papers, 49 (11) (2002), 2075-2093.

[94] Suárez, F., Tyler, S. W., and Childress, A. E., A fully coupled, transient doublediffusive convective model for salt-gradient solar ponds, Int. J. Heat Mass Transf., 53 (910) (2010), 1718-1730.

[95] Montel, F., Phase equilibria needs for petroleum exploration and production industry, Fluid Phase Equilibria, 84(c) (1993), 343-367.

[96] Faissat, B., Knudsen, K., Stenby, E., \& Montel, F., Fundamental statements about thermal diffusion for a multicomponent mixture in a porous medium, Fluid Phase Equilibria, 100 (C) (1994), 209-222.

[97] Kohler, W. E. \& Halbritter, J., Kinetic theory of thermal diffusion in a magnetic field, Zeitschrift fur Naturforschung, 30A (9) (1975), 1114-1121.

[98] Kox, A. J., Van Leeuwen, W. A., \& De Groot S. R., On relativistic kinetic gas theory. XVII. Diffusion and thermal diffusion in a binary mixture of hard spheres, Physica A, 84A(1) (1976), 165-174.

[99] Firoozabadi, A., Ghorayeb, K., \& Shukla, K., Theoretical model of thermal diffusion factors in multicomponent mixtures, AIChE J., 46 (5) (2000), 892-900.

[100] Eslamian, M. \& Saghir, M. Z., Estimation of thermodiffusion coefficients in ternary associating mixtures, Can. J. Chem. Eng., 90(4) (2012), 936-943.

[101] Srinivasan, S. \& Saghir, M. Z., Thermodiffusion in ternary hydrocarbon mixtures: Part 1 - n-dodecane/isobutylbenzene/Tetralin, J. Non-Equil. Thermody., 36 (3) (2011), $243-258$. 
[102] Srinivasan, S. \& Saghir, M. Z., Thermodiffusion in ternary hydrocarbon mixtures: Part 2 - n-dodecane/isobutylbenzene/Tetralin, J. Non-Equil. Thermody., 37 (1) (2012), $99-113$.

[103] Bielenberg, J. \& Brenner, H., A hydrodynamic/brownian motion model of thermal diffusion in liquids, Physica A, 356 (2-4) (2005), 279-293.

[104] Brenner, H., Elementary model of thermal diffusion in liquids and gases, Phys. Rev. E, 74 (3) (2006), 036306-1-20.

[105] Srinivasan, S. \& Saghir, M. Z., Impact of the vibrations on Soret separation in binary and ternary mixtures, Fluid Dynamics and Material Processing, 7 (2) (2011), 201216.

[106] Srinivasan, S. \& Saghir, M. Z., 2010, Thermo-solutal-diffusion in high pressure liquid mixtures in the presence of micro-vibrations, Int. J. Therm. Sci., 49 (9) (2010), 1613-1624.

[107] Parsa, A., Srinivasan, S. \& Saghir, M. Z., Impact of density gradients on the fluid flow inside a vibrating cavity subjected to Soret effect, Can. J. Chem. Eng., 91 (3) (2013), $550-559$.

[108] Srinivasan, S., \& Saghir, M. Z., Predicting thermodiffusion in an arbitrary binary liquid hydrocarbon mixtures using artificial neural networks, Neural Comput. Appl., 25 (5) (2014), 1193-1203.

[109] Srinivasan, S., \& Saghir, M. Z., Estimating the thermotransport factor in binary metal alloys using artificial neural networks, Appl. Math Modeling, (37) (5) 2012, 28502869. 
[110] Schoen, M. \& Hoheisel, C., The mutual diffusion coefficient $\mathrm{D}_{12}$ in liquid model mixture- a molecular dynamics study based on Lennard-jones (12-6) potentials. II. Lorentz-Berthelot mixtures, Mol. Phys., 52 (5) (1984), 1029-1042.

[111] Babaei, H., Keblinski, P., \& Khodadadi, J. M., Equilibrium molecular dynamics determination of thermal conductivity for multi- component systems, J. Appl. Phys., 112 (5) (2012), 054310-1-5.

[112] Wheeler, D. R., Fuller, N. G., \& Rowley, R. L., Non-equilibrium molecular dynamics simulation of the shear viscosity of liquid methanol: Adaptation of Ewald sum to Lees_Edwards bounday conditions, Mol. Phys., 92 (1) (1997), 55-62.

[113] Alaghemandi, M., Algaer, E., Bohm, M. C., \& Müller-Plathe, F., The thermal conductivity and thermal rectification of carbon nanotubes studied using reverse nonequilibrium molecular dynamics simulations," Nanotechnology, 20 (11) (2012), 115704$1-8$.

[114] Guevara-Carrion, G., Vrabec, J., \& Hasse, H., Prediction of transport properties of liquid ammonia and its binary mixture with methanol by molecular simulation, Int. J. Thermophys., 33 (3) (2012), 449-468.

[115] Paolini G. V., \& Ciccotti G., Cross thermotransport in liquid mixtures by non_equilibrium molecular dynamics, Phys. Rev. A, 35 (12) (1987), 5156-5166.

[116] Evans, D. J., Homogeneous NEMD algorithm for thermal conductivity- application of non- canonical linear response theory, Phys. Lett. A, 19 (9) (1982), 457-460.

[117] Müller-Plathe F. \& D. Reith, D., Cause and effect reversed in non-equilibrium molecular dynamics: an easy route to transport coefficients, Comput Theoretical Polymer Sci., 9 (3-4) (199), 203-209. 
[118] Galliero, G., \& Volz, S., Thermodiffusion in model nanofluids by molecular dynamics simulations, J. Chem. Phys., 128 (6) (2008), 064505-1-8.

[119] Galliero, G., \& Montel, F., Nonisothermal gravitational segregation by molecular dynamics simulations, Phys. Rev. E, 78 (4) (2008), 041203-1-10.

[120] De Groot S. R. \& Mazur P., Thermodiffusion in Multicomponent Mixtures, Dover Publications, New York, 1961.

[121] Bird, R. B., Stewart, W. E., \& Lightfoot, E. N., Transport Phenomena, John Wiley \& Sons Publication, 2007.

[122] Allen M. P., \& Tildesley D. J, Computer Simulation of Liquids, Oxford Science Publication, 1987.

[123] Huber, M. L., NIST Thermophysical Properties of Hydrocarbon Mixtures Database, SUPERTRAPP Software, Version 3.2 (1987).

[124] Miller, N. A. T., Daivis, P. J., Snook, I. K., \& Todd, B. D., Computation of thermodynamic and transport properties to predict thermophoretic effects in an argonkrypton mixture, J. Chem. Phys., 139 (2013), 144504-1-144504-10.

[125] Srinivasan S., de Mezquia D. A., Bou-Ali M. M. \& Saghir M. Z., Thermodiffusion and molecular diffusion in binary n-alkane mixtures: Experiments and numerical analysis, Philos. Mag., 91(34) (2011), 4332-4344.

[126] Perronace, A., Leppla, C., Leroy, F., Rousseau, B., \& Wiegand, S., Soret and mass diffusion measurements and molecular dynamics simulations of n- pentane - n-decane mixtures, J. Chem. Phys., 116 (9) (2002), 3718-3729. 
[127] Braun, D., \& Libchaber, A., Trapping of DNA by thermophoretic depletion and convection, Phys. Rev. Lett., 89 (18) (2002), 2-5.

[128] Eslamian, M., \& Saghir, M. Z., Modeling of thermodiffusion in liquid metal alloys, J. Phys. Chem. 12 (41) (2010), 13835-13848.

[129] Richter, F. M., Watson, E. B., Mendybaev, R. A., Teng, F., \& Janney, P. E., Magnesium isotope fractionation in silicate melts by chemical and thermal diffusion, Geochimica et Cosmochimica Acta, 72 (1) (2008), 206-220.

[130] Luettmer-Strathmann, J., Lattice Model for thermodiffusion in polymer, Int. Thermophys., 26 (6) (2005), 1693-1707.

[131] Ahadi, A., \& Ziad Saghir, The microgravity DSC-DCMIX1 mission onboard ISS: experiment description and results on the measurement of the soret coefficients for isobutylbenzene, dodecane, tetralin ternary hydrocarbons mixtures, Exp. Therm. Fluid Sci., 74 (2016), 296-307.

[132] Bataller, H., Giraudet, C., Croccolo, F., Analysis of non-equilibrium fluctuations in a ternary liquid mixture, Microgravity Sci. Technol., 28 (16) (2016), 611-619.

[133] Ahadi, A., \& Saghir, M. Z., Transient effect of micro vibration from two space vehicles on mixture during thermodiffusion experiment, Microgravity Sci. Technol., (25) (2) (2013), 127-139.

[134] Srinivasan, S., \& Saghir, M. Z., Measurements on thermodiffusion in ternary hydrocarbon mixtures at high pressure, J. Chem. Phys., 131 (12) (2009), 1245081-1-8.

[135] Galliero, G., Bataller, H., Croccolo, F., Vermorel, R., Bernard, P. A., Velisa, R., \& Mounir, V., Impact of thermodiffusion on the initial vertical distribution of species in hydrocarbon reservoirs, Microgravity Sci. Technol., 28 (2) (2016), 79-86. 
[136] Tucker, W. C., \& Schelling, P. K., Thermodiffusion in liquid binary alloys computed from molecular dynamics and green-kubo formalism palladium, Comput. Mater. Sci. Phys., 124 (54-61) (2016), 063509-1-8.

[137] Yeganegi, S., \& Kameli, P., Thermal diffusion factor of Stockmayer mixtures : A non-equilibrium molecular dynamic study. J. Chem. Phys., 415 (2013), 119-123.

[138] Galliéro, G., Boned, C., and Baylaucq, A., Molecular dynamics study of the Lennard-Jones fluid viscosity: Application to real fluids, Ind. Eng. Chem. Res., 44 (2005), 6963-6972.

[139] Mozaffari, S. H., Srinivasan, S., \& Saghir, M. Z., Thermodiffusion in binary and ternary hydrocarbon mixtures studied using a modified heat exchange algorithm, accepted in J. Therm. Sci. Eng. Progress,4, 168-174.

[140] Dominguez, G., Wilkins, G., \& Thiemens, M. H., The Soret Effect and isotope fractionation in high-temperature silicate melts, Nature, 36 (7345) (2011), 259-272.

[141] Biala, K., Sedova, A., \& Fichsig, G., Sequence and temperature influence on kinetics of DNA strand displacement at gold electrode, ACS Appl. Mater. Interfaces, 7(36) (2015), 1948-1959.

[142] Messaud, F. A., Sanderson, R. D., Runyon, J. R., Otte, T., Pasch, \& Williams, S. K. R., An overview on field-flow fractionation techniques and their applications in separation and characterization of polymers, Prog. Polym. Sci., 34 (4) (2009), 351-368.

[143] Yan, Y., Pan, S., Jules, K., \& Saghir, M. Z., Vibrational effect on thermal diffusion under different microgravity environment, Microgravity Sci. Technol., 19 (2) (2007),1225 .

[144] Torres, J. F., Komiya, A., Henry, D., \& Maruyama, S., Measurement of Soret and Fickian diffusion coefficients by orthogonal phase-shifting interferometry and its application to protein aqueous solutions, J. Chem. Phys., 139 (7) (2013), 074203-1-21. 
[145] Pham, Q. T., Modelling heat and mass transfer in frozen foods: A review, Int. J. Refrigeration, 29 (6) (2006), 876-888.

[146] Duba, C. T., Shekar, M., Narayana, M., \& Sibanda, P., Soret and dufour effects on thermohaline convection in rotating fluids, Geophysical \& Astrophysical Fluid Dynamics, 110 (4) (2016), 317-347.

[147] Leonardi, E., \& Angeli, C., The effect of thermodiffusion on stability of a salinity gradient solar pond, Int. J. Heat Mass Transf., 48 (21-22) (2005), 4633-4639.

[148] Fargue, D., Costeseque, P., Jamet, Ph.,\& Girard-Gaillard, S., Separation in vertical temperature gradient packed thermodiffusion cells: An unexpected physical explanation to a controversial experimental problem, Chem. Eng. Sci., 59 (24) (2004), 5847-5852.

[149] Blanco, P., Bou-Ali, M., Platten, J. K., Madariaga, A. J., Urteaga, P., \& Santamaria, C., Thermodiffusion coefficient for binary liquid hydrocarbon mixtures, J. Non-Equil. Thermody., 32 (3) (2007), 309-317.

[150] Urteaga, P., Bou-Ali, M., Alsono de Mezquia, D., Santamaria, A. J., Santamaria, A. C., Madariaga, J. A., \& Bataller, H., Measurement of thermodiffusion coefficient of hydrocarbon binary mixtures under pressure with the thermogravitational technique, Rev. Sci. Instrum., 83 (7) (2012), 074903-1-7.

[151] Slavtchev, S., Simeonov, G., Van Vaerenbergh, S, \& Legros, J. C., Marangoni instability of a layer of binary liquid in the presence of nonlinear Soret effect, Int. J. Heat Mass Transf., 42 (15) (1999), 3307-3311.

[152] Eslamian, M., Advanced in thermodiffusion and thermophoresis (Soret effect) in liquid mixtures, Frontiers in Heat Mass Tranf., 2 (4) (2011), 1-20.

[153] Zhan Bang, K., Variational principles for generalized thermodiffusion theory in pyroelecltricity, Acta Mechanica, 214 (3-4) (2010), 275-289. 
[154] Kubik, J., \& Wyrwal, J., Variational principle for linear coupled dynamic theory of viscoelastic thermodiffusion, Int. J. Eng. Sci., 27 (5) (1989), 605-607.

[155] Duziak, W., \& Kowalski, S. J., Theory of thermodiffusion for solids, Int. J. Heat Mass Tranf., 32 (11) (1989), 2005-2013.

[156] Kattoura, S., Lamoureux, A., \& Baliga, B. R., A control volume finite element method for the prediction of three-dimensional diffusion type phenomena in anisotropic media, Comput. Therm. Sic., 5 (3) (2013), 249-260.

[157] Galliero, G., Bataller, H., Croccolo, F., Vermorel, R., Artola, P. A., Rousseau, B., Vesovic, V., Bou-Ali, M., Ortiz de Zearte, J. M., Shenghua, X.,Zhang, K., \& Montel, F., Impact of thermodiffusion on the initial vertical distribution of species in hydrocarbon reservoirs, J. Microgravity Sci. Technol., 28 (2) (2016), 79-86.

[158] Kuang, S., \& Gezelter, J. D., Velocity shearing and scaling RNEMD: A minimally perturbing method for simulating temperature and momentum gradients, Mol. Phys., 110 (9-10) (2012), 691-701.

[159] Wang, B. Y., \& Cummings P. T., Non-Equilibrium molecular dynamics calculation of the transport properties of carbon dioxide, Fluid phase Equilibria, 53 (2) (1989), 191198. 